\title{
All orders results for self-crossing Wilson loops mimicking double parton scattering
}

\author{
Lance J. Dixon ${ }^{a, b}$ and Ilya Esterlis ${ }^{a}$ \\ a SLAC National Accelerator Laboratory, Stanford University, \\ Stanford, CA 94309, U.S.A. \\ ${ }^{b}$ Walter Burke Institute for Theoretical Physics, California Institute of Technology, \\ 1200 E. California Blvd, Pasadena, CA 91125, U.S.A.
}

E-mail: lance@slac.stanford.edu, ilyae@stanford.edu

ABSTRACT: Loop-level scattering amplitudes for massless particles have singularities in regions where tree amplitudes are perfectly smooth. For example, a $2 \rightarrow 4$ gluon scattering process has a singularity in which each incoming gluon splits into a pair of gluons, followed by a pair of $2 \rightarrow 2$ collisions between the gluon pairs. This singularity mimics double parton scattering because it occurs when the transverse momentum of a pair of outgoing gluons vanishes. The singularity is logarithmic at fixed order in perturbation theory. We exploit the duality between scattering amplitudes and polygonal Wilson loops to study six-point amplitudes in this limit to high loop order in planar $\mathcal{N}=4$ super-Yang-Mills theory. The singular configuration corresponds to the limit in which a hexagonal Wilson loop develops a self-crossing. The singular terms are governed by an evolution equation, in which the hexagon mixes into a pair of boxes; the mixing back is suppressed in the planar (large $N_{c}$ ) limit. Because the kinematic dependence of the box Wilson loops is dictated by (dual) conformal invariance, the complete kinematic dependence of the singular terms for the self-crossing hexagon on the one nonsingular variable is determined to all loop orders. The complete logarithmic dependence on the singular variable can be obtained through nine loops, up to a couple of constants, using a correspondence with the multi-Regge limit. As a byproduct, we obtain a simple formula for the leading logs to all loop orders. We also show that, although the MHV six-gluon amplitude is singular, remarkably, the transcendental functions entering the non-MHV amplitude are finite in the same limit, at least through four loops.

KeYwords: 1/N Expansion, Scattering Amplitudes, Supersymmetric gauge theory

ARXIV EPRINT: 1602.02107 


\section{Contents}

1 Introduction $\quad 2$

2 Preliminaries $\quad 6$

$\begin{array}{ll}2.1 \text { Helicity selection rules } & 7\end{array}$

2.2 The BDS-like normalized amplitude 9

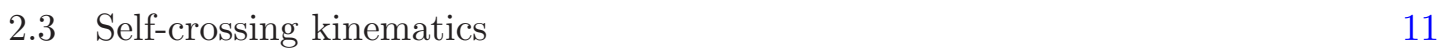

2.4 Framed Wilson loops 12

3 Explicit results through five loops $\quad 16$

3.1 Analytic continuation from Euclidean to $2 \rightarrow 4$ kinematics 16

3.2 From $2 \rightarrow 4$ to $3 \rightarrow 3$ kinematics with $v<0 \quad 19$

3.3 A few observations 21

3.4 From $v<0$ to $v>1 \quad 22$

$3.5 \quad v \rightarrow \infty$ and $v \rightarrow 1$ limits 23

4 Evolving framed self-crossing Wilson loops $\quad 25$

4.1 A simple evolution equation 25

$\begin{array}{lll}4.2 & \text { The large } \gamma \text { limit } & 27\end{array}$

4.3 The framed double box 28

5 Matching the self-crossing and multi-Regge limits $\quad 30$

5.1 Self-crossing-MRK limit of the Wilson loop in $3 \rightarrow 3$ kinematics 31

5.2 Evaluation of the Fourier-Mellin transform for $w \rightarrow-1$

$6 \quad$ Final result for singular terms $\quad 35$

$\begin{array}{llr}7 & \text { Conclusions and outlook } & 38\end{array}$

$\begin{array}{lr}\text { A Self-crossing kinematics } & 39\end{array}$

$\begin{array}{lll}\text { A.1 } 2 \rightarrow 4 \text { kinematics } & 39\end{array}$

A.2 $3 \rightarrow 3$ kinematics $\quad 42$

B Perturbative expansion of cusp anomalous dimension 45

C Four- and five-loop results for the MHV amplitude in the $3 \rightarrow 3$ selfcrossing limit for $v>0 \quad 46$

D NMHV results in the $3 \rightarrow 3$ self-crossing limit for $v>0$ through four loops 


\section{Introduction}

At high-energy hadron colliders such as the Tevatron and the Large Hadron Collider, double parton scattering can take place, in which two partons from each incoming hadron collide with each other. The kinematic signature of such an event is that the final state can be split into two subsets of constituents, which are the products of the two separate partonic collisions. The transverse momentum of each subset should add up to zero, whereas for a single parton scattering with the same final state this is not generically true. Single-parton tree amplitudes are smooth as one approaches the kinematics of double-parton scattering. However, at loop level the single-parton scattering amplitude can have a logarithmic singularity in the subset transverse momentum, which can be identified with a Landau or pinch singularity in the Feynman parameter integration [1-9]. This singularity arises because, at the loop level, each incoming parton can split into two collinear partons, each of which then participates in a scattering.

Figure 1(a) shows such a configuration for massless $2 \rightarrow 4$ scattering, where particles 3 and 6 are incoming, and particles 1, 2, 4 and 5 are outgoing. This configuration is generically singular as the vector sum of the transverse momenta of particles 1 and 2 vanishes, because of the existence of the $2 \rightarrow 2$ subprocesses $(1-x) k_{3}+(1-y) k_{6} \rightarrow k_{1}+k_{2}$ and $x k_{3}+y k_{6} \rightarrow k_{4}+k_{5}$. The lines marked $x,(1-x)(y,(1-y))$ in the figure can go on-shell in this limit; they are approximately collinear to the initial-state particle 3 (particle 6 ), and they contain the indicated fraction of its longitudinal momentum. Similar configurations arise for $2 \rightarrow(n-2)$ processes for any $n \geq 6$, although in this paper we will mostly study the six-point amplitude.

Although of less direct phenomenological relevance, there are similar singularities for scattering processes with more than two initial particles. For the case of the six-point amplitude, figure 1(b) shows such a singularity in $3 \rightarrow 3$ scattering. An initial parton splits into two collinear partons, each of which collides with another incoming parton; two of the products of those collisions then fuse into a single parton. Interestingly, at least for the theory we will be considering in this paper, the $3 \rightarrow 3$ process has a somewhat simpler structure in the singular limit, and the $2 \rightarrow 4$ case can then be obtained from it by an analytic continuation.

In the experimental effort to isolate hard multiple parton interactions, the background from single-parton contributions in the same region of phase space must be subtracted, usually by extrapolation from data with finite values of the subset transverse momentum. In the case of jet final states, the jet energy resolution typically smears the double-parton events over a range of finite subset transverse momenta, and the expected "bump" at zero may become a broad shoulder. Hence it is of some interest to understand any nonsmooth behavior of the single-parton background. Because the matrix element singularity is merely logarithmic, the phase-space integration over the subset transverse momentum is convergent. However, the detailed shape as one approaches zero may still be important. It 


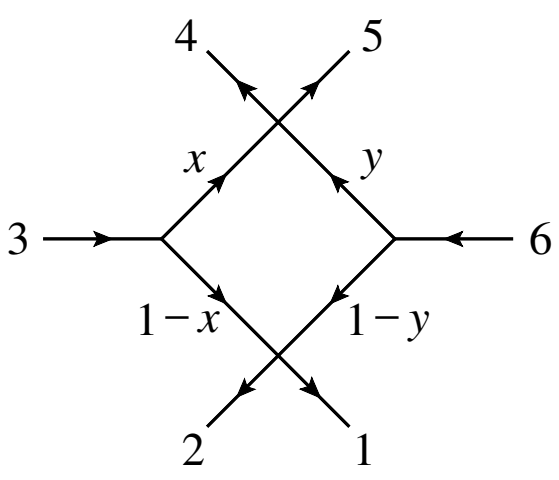

(a)

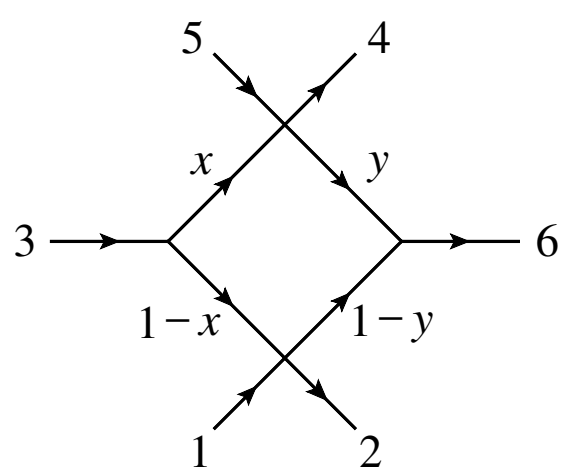

(b)

Figure 1. (a) A $2 \rightarrow 4$ scattering configuration mimicking double-parton scattering. Incoming gluons 3 and 6 split into collinear pairs with very small transverse momentum, and longitudinal momentum fractions $x$ and $1-x$, and $y$ and $1-y$, respectively. These pairs then undergo $2 \rightarrow 2$ scatterings into final state gluons 1, 2, 4 and 5 . (b) The analogous configuration for $3 \rightarrow 3$ scattering. Gluons 1 , 3 and 5 are incoming. Gluon 3 splits collinearly, and its daughters collide with gluons 1 and 5 . These $2 \rightarrow 2$ collisions produce gluons 2 and 4 , and two more gluons which fuse collinearly into gluon 6 .

is not our purpose in this paper to perform any phenomenological discussion of the singleparton contributions to double parton scattering or how to separate them theoretically (see e.g. refs. $[8,10-16])$. Rather, we would like to use a toy model to study the behavior of scattering amplitudes in this region to very high perturbative order.

The toy model we have in mind is $\mathcal{N}=4$ super-Yang-Mills theory (SYM) [17] in the limit of a large number of colors $N_{c}$. In this limit, the theory is integrable [18] and possesses a dual superconformal symmetry [19-23]. Perhaps most interestingly for the present problem, scattering amplitudes in planar $\mathcal{N}=4 \mathrm{SYM}$ are dual to polygonal Wilson loops with light-like edges [22, 24-27]. Each edge of the Wilson loop corresponds to an external momentum of the scattering amplitude, and the closure of the polygon is the geometrical statement of overall momentum conservation.

The Wilson loop configuration that mimics double-parton scattering is one in which the loop crosses itself, as shown in figure 2. Comparing this figure with figure 1, we see that the splitting of particle 3 into two collinear intermediate particles with momentum fractions $x$ and $1-x$ is reflected in the division of line 3 in figure 2 into two segments labelled by $x$ and $1-x$, and similarly for particle 6 with momentum fractions $y$ and $1-y$. The reason lines 3 and 6 touch (in the singular limit) is to ensure momentum conservation of the subprocesses $(1-x) k_{3}+(1-y) k_{6} \rightarrow k_{1}+k_{2}$ and $x k_{3}+y k_{6} \rightarrow k_{4}+k_{5}$ (or their analytic continuation in the case of $3 \rightarrow 3$ scattering). For convenience, we will refer to this kinematics as "self-crossing", even when we discuss theories other than planar $\mathcal{N}=4$ SYM for which there is no polygonal Wilson loop correspondence, and where the term "double-parton-scattering-like" might be more appropriate.

The behavior of the self-crossing hexagonal Wilson loop in planar $\mathcal{N}=4 \mathrm{SYM}$ has been studied by Georgiou [28] at two loops, and by Dorn and Wuttke [29, 30] at two and 


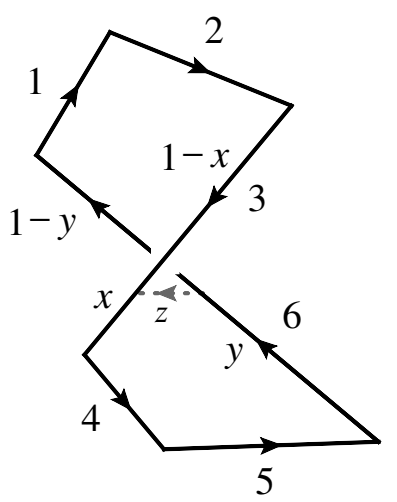

Figure 2. Self-crossing configuration of a hexagonal Wilson loop, regulated by a small space-like vector $\vec{z}$.

three loops. This "bosonic" Wilson loop corresponds to the six-gluon maximally helicity violating (MHV) scattering amplitude, in which the gluons have the (all-outgoing) helicity configuration $(--++++)$, or any any permutation thereof. The self-crossing configuration depends on one singular parameter, $\delta \ll 1$. This parameter is invariant under the dual conformal symmetry possessed by Wilson loops in planar $\mathcal{N}=4$ SYM. It serves as a proxy for the vanishing subset transverse momentum. In figure 2 , we regulate the self-crossing singularity by a small space-like separation vector $\vec{z}[31,32]$. We will see that the magnitude of the separation, $\vec{z}^{2}$, is proportional to $\delta$.

The self-crossing configuration depends as well on one generic, nonsingular parameter we call $v$. In this paper we will determine how the singular ( $\ln \delta$ containing) terms in this Wilson loop (amplitude) depend on $v$ to all loop orders. We will present the full logarithmic dependence on $\delta$ through seven loops, and at eight and nine loops up to a couple of constants. We'll also give the full $v$ dependence of the nonsingular terms through five loops, neglecting terms suppressed by powers of $\delta$.

We will show that the transcendental functions entering the other six-point helicity configuration in $\mathcal{N}=4 \mathrm{SYM}$, called non-MHV (NMHV), are actually nonsingular through four loops! This result is related to an argument of Gaunt and Stirling for one-loop QED amplitudes [7].

In order to provide explicit MHV results to such a high loop order, we will make use of the factorized singularity structure of Wilson loops that are close to crossing [33, 34], in particular the analysis and evolution equation studied by Korchemskaya and Korchemsky $[31,32]$. As one approaches the singularity, the hexagonal Wilson loop mixes with another configuration, which features two disconnected squares corresponding to the two $2 \rightarrow 2$ subprocesses. At large $N_{c}$, the mixing of the two-square configuration back into the hexagon is suppressed, and the expectation value of the two-square Wilson loop is dictated by dual conformal invariance. This leads to an exact prediction for how the singular terms in the hexagonal Wilson loop depend on the unique nonsingular kinematic variable, $v$.

Knowing the full dependence on $v$ for the singular terms, we can evaluate them by choosing $v$ to be anything we like. We make use of the fact that as $v \rightarrow 0$, the self-crossing 
limit overlaps with the limit of multi-Regge kinematics (MRK), which has been studied extensively in planar $\mathcal{N}=4 \mathrm{SYM}$ [35-52]. In particular, an all-orders formula for the behavior of the Wilson loop in this limit was proposed by Basso, Caron-Huot and Sever (BCS) [49], based on integrability and an analytic continuation from the Euclidean operator product expansion region studied by Basso, Sever and Vieira [53-56]. We will analyze this formula to high loop orders in the region of overlap with the self-crossing configuration, and make use of recent high order results by Drummond and Papathanasiou [50] and especially by Broedel and Sprenger [51]. The exact $v$ dependence provides cross checks on the MRK predictions of BCS.

Finally, we have used a very recent determination of the full MHV amplitude at five loops [57], as well as lower-loop results [41, 47, 58, 59], to obtain the full $v$ dependence of the nonsingular terms through this order. We also present the nonsingular limits of the transcendental functions entering the NMHV amplitude through four loops, using results from refs. [60-62].

Similar methods for controlling the singular terms should be applicable as well to higher-point amplitudes, but we leave that for future work.

This paper is organized as follows: section 2 reviews properties of amplitudes and Wilson loops in planar $\mathcal{N}=4 \mathrm{SYM}$. It describes the self-crossing limit and explains why the transcendental functions in NMHV six-gluon amplitudes are expected to be nonsingular there, while MHV amplitudes diverge. Finally, it discusses how to frame Wilson loops to remove their cusp divergences, and how different framings behave in the self crossing limit. Section 3 provides explicit results for the MHV amplitude through five loops, after discussing how to analytically continue into these regions. It also looks at a few special limits $(v \rightarrow \infty$ and $v \rightarrow 1)$ where the results simplify.

Section 4 discusses how the singular terms as $\delta \rightarrow 0$ obey an evolution equation, one that is particularly simple due to the large $N_{c}$ limit. This equation explains several properties of the explicit results, and allows one to go to higher loop order using the $v \rightarrow 0$ limit. Section 5 shows how the $v \rightarrow 0$ limit of self-crossing overlaps with the $w \rightarrow-1$ limit of multi-Regge-kinematics. It also develops techniques for evaluating the Fourier-Mellin transform in this limit, and discusses a comparison with information from ref. [51]. Section 6 presents the final expression for the self-crossing limit, and organizes the dependence on $\delta$ into a suggestive form, so that no pure even $\zeta$ values appear explicitly, and particular terms with single odd $\zeta$ values are confined to a specific dependence on $\ln |\delta|$. Finally, in section 7 we conclude.

We also provide multiple appendices. In appendix A we give a detailed description of both $2 \rightarrow 4$ and $3 \rightarrow 3$ self-crossing kinematics in terms of the kinematics of the $2 \rightarrow 2$ subprocesses. Appendix B gives the expansion of the light-like cusp anomalous dimension through 10 loops. Appendix $\mathrm{C}$ gives the full four- and five-loop results for the MHV amplitude in the self-crossing limit, while appendix D presents results for the nonsingular NMHV transcendental functions through four loops. Appendix E gives a brief description of the self-crossing limit for the seven-point case, which will be explored more thoroughly in future work.

Accompanying this article is an ancillary file containing computer-readable expressions for the lengthier formulae in this paper. 


\section{Preliminaries}

As mentioned in the introduction, scattering amplitudes in planar $\mathcal{N}=4 \mathrm{SYM}$ are dual to polygonal Wilson loops. For the MHV $n$-gluon amplitude, where two gluon helicities are negative and the rest positive, the correspondence is via

$$
A_{n}^{\mathrm{MHV}}=A_{n}^{\mathrm{MHV}, \text { tree }} W_{n}=A_{n}^{\mathrm{BDS}}\left(s_{i, j} ; \epsilon\right) \exp \left[R_{n}\left(u_{i j k l}\right)\right]
$$

where $A_{n}^{\mathrm{MHV}}$ is the partial amplitude associated with the color factor $\operatorname{Tr}\left(T^{a_{1}} T^{a_{2}} \ldots T^{a_{n}}\right)$, $A_{n}^{\mathrm{MHV} \text {, tree }}$ is the corresponding tree-level amplitude, $W_{n}$ is the Wilson $n$-gon expectation value, $A_{n}^{\mathrm{BDS}}$ is the BDS ansatz [63], and $R_{n}$ is the remainder function which corrects

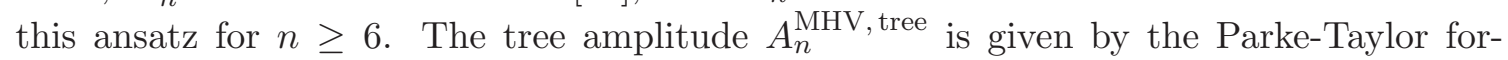
mula $[64,65]$,

$$
A_{n}^{\mathrm{MHV}, \text { tree }}=i \frac{\langle j k\rangle^{4}}{\langle 12\rangle\langle 23\rangle \cdots\langle n 1\rangle}
$$

where $j$ and $k$ label the two negative-helicity gluons, in the all-outgoing helicity convention. It is important to note that all of the dependence on $j$ and $k$ is carried by the simple prefactor $\langle j k\rangle^{4}$. The more complicated quantity, the bosonic Wilson loop, carries no helicity information at all.

The BDS ansatz depends on the Mandelstam variables $s_{i j}$ and is given by

$$
A_{n}^{\mathrm{BDS}}=A_{n}^{\mathrm{MHV}, \text { tree }} \exp \left[\sum_{L=1}^{\infty} a^{L}\left(f^{(L)}(\epsilon) \frac{1}{2} M_{n}^{1-\mathrm{loop}}(L \epsilon)+C^{(L)}\right)\right],
$$

where $M_{n}^{1-\text { loop }}(L \epsilon)$ is the one-loop amplitude, normalized by the tree amplitude, and evaluated in dimensional regularization with $D=4-2 \epsilon$, but letting $\epsilon \rightarrow L \epsilon$. The remaining quantities in eq. (2.3) are constants:

$$
f^{(L)}(\epsilon) \equiv f_{0}^{(L)}+\epsilon f_{1}^{(L)}+\epsilon^{2} f_{2}^{(L)},
$$

where two of the constants,

$$
f_{0}^{(L)}=\frac{1}{4} \gamma_{K}^{(L)}, \quad f_{1}^{(L)}=\frac{L}{2} \mathcal{G}_{0}^{(L)},
$$

are given in terms of the (light-like) cusp anomalous dimension $\gamma_{K}$ and the "collinear" anomalous dimension $\mathcal{G}_{0}$, and $f_{2}^{(L)}$ and $C^{(L)}$ are other constants, known analytically to three loops. The BDS ansatz captures all the infrared singularities of the scattering amplitude, or equivalently the ultraviolet cusp singularities of the Wilson loop. However, there is a simpler, "BDS-like" ansatz that also does this, which we will introduce in section 2.2.

We should note that eq. (2.1) is formal due to infrared divergences. In fact the $1 / \epsilon$ pole in the logarithm of the Wilson loop is controlled by $\mathcal{G}_{\text {eik }}$, a quantity that differs from $\mathcal{G}_{0}$ by a term proportional to the virtual part of the DGLAP kernel [66-68]. This difference will not matter below, once we pass to finite quantities on both sides of the duality. 


\subsection{Helicity selection rules}

Because the MHV tree amplitude (2.2) carries all the helicity dependence of the full MHV amplitude, we can immediately make some all-order statements about how the self-crossing result in $\mathcal{N}=4 \mathrm{SYM}$ depends on the helicity configuration. The spinor products $\langle i j\rangle$ entering eq. (2.2) are all nonsingular and finite in the generic self-crossing configuration. Hence there are at most simple finite factors between the different helicity configurations, and all will have the same singularities in the self-crossing limit. We can contrast this behavior with that of the one-loop QED six-photon amplitude with an electron in the loop, as analyzed in ref. [7]. In that case, non-singular self-crossing limits of certain helicity configurations appear for two reasons:

1. If two of the outgoing photons from a $2 \rightarrow 2$ subprocess have the same helicity, then the tree amplitude for $e^{+} e^{-} \rightarrow \gamma^{+} \gamma^{+}$vanishes for massless electrons, causing the one-loop amplitude to be nonsingular.

2. If the two incoming photons have opposite helicities, then the $J_{z}$ of the initial state is nonzero; however, by helicity conservation for massless electrons, the four-electron intermediate state has $J_{z}=0$.

As a result of these selection rules, the only possible singular MHV configurations for six photons at one loop are $++\rightarrow(-+)(-+)$, where we use all-outgoing helicity labelings, and separate the pairs of final-state photons for the two $2 \rightarrow 2$ subprocesses using parentheses. In addition, there are no singular NMHV configurations.

The difference between one-loop QCD or planar $\mathcal{N}=4$ SYM amplitudes with gluons circulating in the loop, and one-loop QED amplitudes, is that gluons can have a helicity flip while circulating around the loop, and therefore the outgoing gluons from a $2 \rightarrow 2$ subprocess can have the same helicity, in contrast to selection rule 1. Massless quarks, gluinos and scalars in the loop obey rule 1, just like massless electrons. However, rule 2, or more generally $J_{z}$ conservation between the initial state and the four-parton intermediate state, is an important constraint that still needs to be applied.

Figure 3 shows four different configurations that satisfy both rules 1 and 2 for gluons circulating in the loop. Only the first one, (a), for $++\rightarrow(-+)(-+)$, appears in QED. Configurations (b), (c) and (d) are forbidden by rule 1 in QED, but permitted in QCD or planar $\mathcal{N}=4 \mathrm{SYM}$ amplitudes. Correspondingly, the MHV configurations $++\rightarrow$ $(--)(++),-+\rightarrow(-+)(++)$ and $--\rightarrow(++)(++)$, although non-singular in QED, are singular in $\mathrm{QCD}$ or $\mathcal{N}=4 \mathrm{SYM}$, because they obey the $J_{z}$ conservation rule. Because only gluons contribute to the singularity, the singular behavior of the one-loop QCD amplitude for these three helicity configurations is identical to that for $\mathcal{N}=4$ SYM studied in this paper, and therefore all three cases are simply related by the helicity dependence of the MHV tree amplitude.

On the other hand, for $++\rightarrow(-+)(-+)$, the QCD and $\mathcal{N}=4$ SYM results are different, because massless quarks, gluinos and scalars can contribute. While the $\mathcal{N}=4$ supersymmetric sum will reconstruct a result for $\mathcal{N}=4 \mathrm{SYM}$ that is simply related to the other MHV configurations, the QCD result will have a different form. 


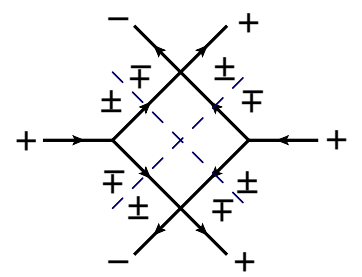

(a)

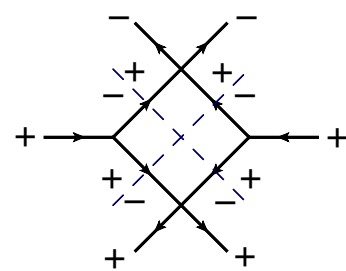

(b)

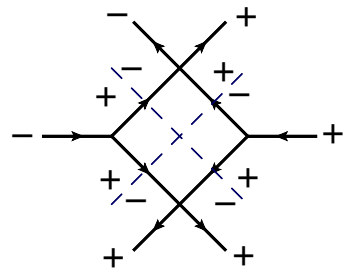

(c)

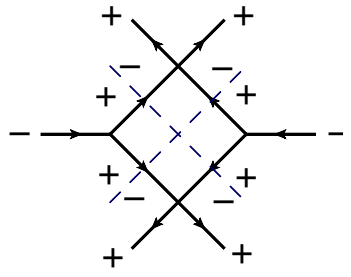

(d)

Figure 3. Allowed helicity configurations for a singular self-crossing limit for the MHV six-gluon amplitude. The cut internal lines are collinear with the incoming particles, as in figure 1. Only (a) is allowed for massless matter circulating in the loop; (b), (c) and (d) require gluons to circulate.

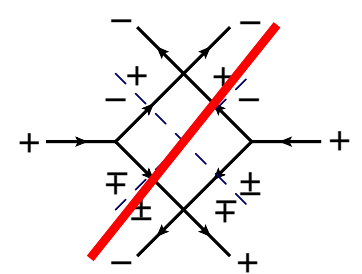

(a)

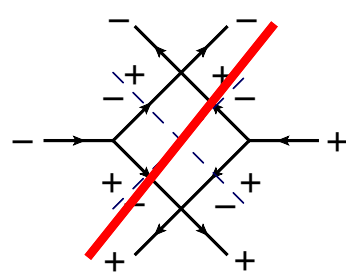

(b)

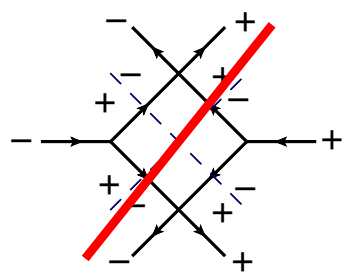

(c)

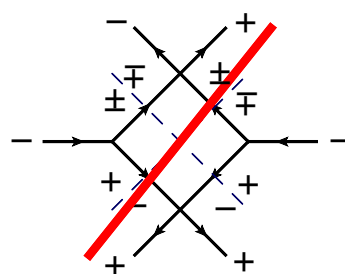

(d)

Figure 4. NMHV six-gluon helicity configurations. All are forbidden to have self-crossing singularities by the $J_{z}$ conservation rule (hence the red lines through them). One (QED-like) configuration for $-+\rightarrow(-+)(-+)$ is not shown; it also violates $J_{z}$ conservation.

What about NMHV helicity configurations? Ref. [7] shows that there are no NMHV helicity configurations in QED (or for any massless matter contribution) that obey rules 1 and 2. Remarkably, even with gluons in the loop, so that rule 1 can be relaxed, rule $2\left(J_{z}\right.$ conservation $)$ still forbids any singular configurations. Figure 4 shows four different helicity configurations that would appear to factorize properly into nonvanishing $1 \rightarrow 2$ splittings and $2 \rightarrow 2$ subprocesses. However, all four of them are non-singular because they violate $J_{z}$ conservation between the initial and the intermediate state. For example, in case (a), for $++\rightarrow(--)(-+)$, the initial $J_{z}$ is zero, but the final $J_{z}$ is \pm 2 . In case (b), for $-+\rightarrow(--)(++)$, the initial $J_{z}$ is \pm 2 , while the final $J_{z}$ is zero. (There is a fifth configuration, not shown, for $-+\rightarrow(-+)(-+)$, which is of the same type allowed by rule 1 ; but it violates $J_{z}$ conservation just as it does in QED.)

Thus, as a consequence of $J_{z}$ conservation, the NMHV six-gluon amplitude is nonsingular in all self-crossing limits, to one-loop in $Q C D$, and in $\mathcal{N}=1$ or $\mathcal{N}=4 S Y M$. Naively, the result should hold to all orders in supersymmetric gauge theories because supersymmetry forbids the "non-tree-like" $2 \rightarrow 2$ helicity amplitudes $(++++)$ and $(-+++)$. That four-point selection rule dictates the configurations shown in figure 4, plus the fifth, QEDlike one, all of which violate $J_{z}$ conservation. At two loops in QCD, the NMHV six-gluon amplitude will presumably become singular; it is easy to write down helicity configurations that satisfy $J_{z}$ conservation once one of the $2 \rightarrow 2$ amplitudes for $(-+++)$ or its parity conjugate $(+---)$ is nonzero. 
The NMHV six-gluon amplitude has been computed in planar $\mathcal{N}=4$ SYM through four loops [60-62]. In appendix D we provide the self-crossing limits of the transcendental functions entering these results. We find that these functions are indeed completely nonsingular in the self-crossing limit through four loops. However, the full NMHV amplitude contains rational function prefactors which blow up like $1 / \sqrt{\delta}$ in the self-crossing limit. Therefore the full amplitude can be singular even if the transcendental functions are finite. To analyze the behavior of the full amplitude requires expanding the transcendental functions to higher order around the self-crossing limit. In appendix D we carry out this expansion, not in the full self-crossing limit, but in the part that overlaps with multi-Reggekinematics. We find logarithmic singularities in $\delta$ starting at two loops. Thus there must be a loophole in the naive argument for all-loop order NMHV finiteness in the self-crossing limit, perhaps from contributions where more than one particle crosses a cut, which can happen starting at two loops. We leave further investigation of this issue to future work.

\subsection{The BDS-like normalized amplitude}

The BDS ansatz also accounts for an anomaly in dual conformal invariance due to the infrared (ultraviolet) divergences of the scattering amplitude (Wilson loop). The remainder function $R_{n}$ is then invariant under dual conformal transformations. Hence $R_{n}$ can only be a function of the dual conformally invariant cross ratios,

$$
u_{i j k l}=\frac{x_{i j}^{2} x_{k l}^{2}}{x_{i k}^{2} x_{j l}^{2}},
$$

where $x_{i j}^{2}=\left(x_{i}-x_{j}\right)^{2}$, the dual coordinates $x_{i}^{\mu}$ describe the locations of the vertices of the $n$-gon, and the scattering amplitude momenta $k_{i}^{\mu}$ are related to them by $k_{i}^{\mu}=x_{i}^{\mu}-x_{i+1}^{\mu}$. Non-trivial cross ratios require non-adjacent vertices, since $x_{i, i+1}^{2}=k_{i}^{2}=0$. There are no such invariants for $n=4$ or 5 , and the remainder function first becomes nonvanishing for $n=6[35,69,70]$.

For $n=6$, the main subject of this paper, there are three independent cross ratios,

$$
\begin{aligned}
& u=u_{1}=\frac{s_{12} s_{45}}{s_{123} s_{345}}=\frac{x_{13}^{2} x_{46}^{2}}{x_{14}^{2} x_{36}^{2}}, \\
& v=u_{2}=\frac{s_{23} s_{56}}{s_{234} s_{123}}=\frac{x_{24}^{2} x_{51}^{2}}{x_{25}^{2} x_{41}^{2}}, \\
& w=u_{3}=\frac{s_{34} s_{61}}{s_{345} s_{234}}=\frac{x_{35}^{2} x_{62}^{2}}{x_{36}^{2} x_{52}^{2}},
\end{aligned}
$$

where $s_{i, i+1}=\left(k_{i}+k_{i+1}\right)^{2}, s_{i, i+1, i+2}=\left(k_{i}+k_{i+1}+k_{i+2}\right)^{2}$, and $x_{i j}^{2} \equiv\left(x_{i}-x_{j}\right)^{2}$.

The remainder function, $R_{6}(u, v, w)$, has a Euclidean branch for which $u, v$ and $w$ are all positive and the function is real. The $2 \rightarrow 4$ and $3 \rightarrow 3$ scattering configurations are physical, Minkowski configurations, which can be obtained from the Euclidean region by a suitable analytic continuation $[28,30,35,39]$. For $2 \rightarrow 4$ scattering, we are interested in the configuration with particles 3 and 6 incoming - see figure 1(a) and eqs. (A.1)-(A.3). This is achieved by letting $u \rightarrow u e^{-2 \pi i}$ and leaving $v$ and $w$ positive. For $3 \rightarrow 3$ scattering, we 
wish to take particles 1, 3 and 5 to be incoming - see figure 1(b) and eqs. (A.30)-(A.32). To do this (for the case $v, w<0$ ), we let $u \rightarrow u e^{+2 \pi i} v \rightarrow v e^{\pi i}, w \rightarrow w e^{\pi i}$. (See also sections 3.1 and 3.2 below.)

Instead of considering the remainder function, which is the (log of) the amplitude normalized by the BDS ansatz, for our present problem it is better to normalize the amplitude by a "BDS-like" ansatz [71]. The reason is that the BDS ansatz contains the one-loop $\mathcal{N}=4 \mathrm{SYM}$ amplitude, which is also singular in the self-crossing limit. The BDS-like ansatz has a simpler functional form, depending only on two-particle invariants, and it is nonsingular in the limit.

More specifically, the BDS-like ansatz for six-gluon scattering is

$$
A_{6}^{\mathrm{BDS}-\mathrm{like}}=A_{6}^{\mathrm{MHV}, \text { tree }} \exp \left[\sum_{L=1}^{\infty} a^{L}\left(f^{(L)}(\epsilon) \frac{1}{2} \hat{M}_{6}(L \epsilon)+C^{(L)}\right)\right],
$$

where

$$
\begin{aligned}
& \hat{M}_{6}(\epsilon)=M_{6}^{1-\text { loop }}+Y(u, v, w) \\
& \quad=\sum_{i=1}^{6}\left[-\frac{1}{\epsilon^{2}}\left(1-\epsilon \ln \left(-s_{i, i+1}\right)\right)-\ln \left(-s_{i, i+1}\right) \ln \left(-s_{i+1, i+2}\right)+\frac{1}{2} \ln \left(-s_{i, i+1}\right) \ln \left(-s_{i+3, i+4}\right)\right] \\
& \quad+6 \zeta_{2},
\end{aligned}
$$

with

$$
Y(u, v, w)=\operatorname{Li}_{2}(1-u)+\operatorname{Li}_{2}(1-v)+\operatorname{Li}_{2}(1-w)+\frac{1}{2}\left(\ln ^{2} u+\ln ^{2} v+\ln ^{2} w\right) .
$$

Our perturbative expansion parameter for planar SYM is $a=g_{\mathrm{YM}}^{2} N_{c} /\left(8 \pi^{2}\right)$, where $N_{c}$ is the (large) number of colors and $g_{\mathrm{YM}}$ is the Yang-Mills coupling. Note that $\hat{M}_{6}(\epsilon)$ contains only two-particle invariants $s_{i, i+1}$, which are just the squares of the spinor products $\langle i, i+$ $1\rangle$ entering the MHV tree amplitude (2.2). Therefore the BDS-like ansatz is completely smooth as one approaches self-crossing kinematics, yet it still removes infrared divergences in a way that respects dual conformal invariance, since it only differs from the BDS ansatz by the dual conformally invariant function $Y(u, v, w)$.

Comparing eqs. (2.3) and (2.8), we see that

$$
A_{6}^{\mathrm{BDS}-\mathrm{like}}=A_{6}^{\mathrm{BDS}} \exp \left[\frac{\gamma_{K}}{8} Y(u, v, w)\right] .
$$

We define the function $\mathcal{E}(u, v, w)$ by

$$
A_{6}^{\mathrm{MHV}}=A_{6}^{\mathrm{BDS}-\mathrm{like}}\left(s_{i, i+1}, \epsilon\right) \times \mathcal{E}(u, v, w) .
$$

Eq. (2.11) shows that it is related to the remainder function by

$$
\mathcal{E}(u, v, w)=\exp \left[R_{6}(u, v, w)-\frac{\gamma_{K}}{8} Y(u, v, w)\right] .
$$

Using integrability, the cusp anomalous dimension $\gamma_{K}(a)$ can be computed to arbitrary loop orders [72]. Its expansion through 10 loops in terms of $a$ is given in appendix B. 
Although our main focus will be on the singularities of the MHV six-gluon amplitude, we will also present the nonsingular limits (see section 2.1) of the transcendental functions entering the NMHV amplitude. As described in more detail in ref. [62], the NMHV superamplitude can be written in a BDS-like form as

$$
\begin{aligned}
\frac{\mathcal{A}_{6}^{\mathrm{NMHV}}}{\mathcal{A}_{6}^{\mathrm{BDS}-\text { like }}} & =\frac{1}{2}[[(1)+(4)] E(u, v, w)+[(2)+(5)] E(v, w, u)+[(3)+(6)] E(w, u, v) \\
& \left.+[(1)-(4)] \tilde{E}\left(y_{u}, y_{v}, y_{w}\right)-[(2)-(5)] \tilde{E}\left(y_{v}, y_{w}, y_{u}\right)+[(3)-(6)] \tilde{E}\left(y_{w}, y_{u}, y_{v}\right)\right],
\end{aligned}
$$

where $\mathcal{A}_{6}^{\mathrm{NMHV}}$ and $\mathcal{A}_{6}^{\mathrm{BDS}-\text { like }}$ are super-amplitudes and $(1),(2), \ldots,(6)$ are shorthand notation for six Grassmann-variable-containing dual-superconformal " $R$ " invariants. The coefficient functions $E$ and $\tilde{E}$ are related to the more conventional components of the ratio function, $V$ and $\tilde{V}$, by

$$
\begin{aligned}
& E(u, v, w)=V(u, v, w) \exp \left[R_{6}(u, v, w)-\frac{\gamma_{K}}{8} Y(u, v, w)\right], \\
& \tilde{E}(u, v, w)=\tilde{V}(u, v, w) \exp \left[R_{6}(u, v, w)-\frac{\gamma_{K}}{8} Y(u, v, w)\right] .
\end{aligned}
$$

Using the quantities $E$ and $\tilde{E}$ also simplifies the global structure of the NMHV amplitude $[61,62]$. Similarly, the MHV amplitude's global structure is most simply expressed in terms of $\mathcal{E}[57]$.

The functions $\tilde{E}$ and $\tilde{V}$ are odd under parity and, like all parity-odd functions, they vanish like a power of $\delta$ as one approaches the self-crossing limit. However, it is very nontrivial that the parity-even function $E(u, v, w)$ remains finite in the limit through four loops, for all different orientations. These finite limits are presented in appendix D.

\subsection{Self-crossing kinematics}

After the analytic continuation to either $2 \rightarrow 4$ or $3 \rightarrow 3$ kinematics, the self-crossing limit constrains two of the cross ratios. The precise relations between the subprocess scattering angles and the kinematic invariants are worked out in appendix A, strictly in the self-crossing limit. From momentum conservation for the $2 \rightarrow 2$ subprocesses, we obtain eqs. (A.5)-(A.10). Inserting these into the definitions of the cross ratios, we have

$$
\begin{aligned}
u & =\frac{s_{12} s_{45}}{s_{123} s_{345}}=\frac{(1-x)(1-y) s_{36} x y s_{36}}{x(1-y) s_{36} y(1-x) s_{36}}=1, \\
\frac{v}{w} & =\frac{s_{23} s_{56} s_{345}}{s_{123} s_{34} s_{61}}=\frac{(1-y) x y(1-x)}{x(1-y) y(1-x)}=1 .
\end{aligned}
$$

Thus there is only one nonsingular variable, $v$, characterizing the self-crossing limit.

We regulate the self-crossing singularity by moving $u$ slightly away from 1 . We rewrite momentum conservation near the self-crossing limit as

$$
\begin{aligned}
k_{1}+k_{2}+(1-x) k_{3}+(1-y) k_{6} & =z \\
k_{4}+k_{5}+x k_{3}+y k_{6} & =-z,
\end{aligned}
$$


where $z^{\mu}$ is a small, space-like vector $z=(0, \vec{z})$ orthogonal to $k_{3}$ and $k_{6}$ (see figure 2 ). Then eqs. (A.5)-(A.8) all acquire an additional term of $-\vec{z}^{2}$ on the right-hand side. Correcting eq. (2.17) for this and expanding to first order in $\vec{z}^{2}$, we see that ${ }^{1}$

$$
u=1-\delta, \quad w=v,
$$

where

$$
\delta=\frac{\vec{z}^{2}}{s_{36} x y(1-x)(1-y)} .
$$

Note that for $2 \rightarrow 4$ kinematics, $s_{36}$ is positive, so $\delta>0$; whereas for $3 \rightarrow 3$ kinematics, $s_{36}$ is negative, so $\delta<0$, and in this case we will let $\delta=-|\delta|$.

Appendix A also indicates the values of $v$ that correspond to $2 \rightarrow 4$ versus $3 \rightarrow 3$ kinematics:

$$
\begin{array}{ll}
2 \rightarrow 4 \text { kinematics: } & (u, v, w)=(1-\delta, v, v), \quad \delta>0, \quad 0<v<1 \\
3 \rightarrow 3 \text { kinematics : } & (u, v, w)=(1+|\delta|, v, v), \quad v<0 \text { and } 1<v .
\end{array}
$$

The range in $v$ for $3 \rightarrow 3$ kinematics splits into two segments because, as explained in appendix A, $v=w=\infty$ corresponds to $s_{234}=0$. The 3 -particle invariant $s_{234}$ can vanish in the interior of phase-space only for $3 \rightarrow 3$ kinematics. It corresponds to the potential factorization pole when a six-point amplitude separates into two four-point amplitudes. (Such a pole is absent in supersymmetric theories in the MHV case, due to helicity selection rules, although it can be there in the NMHV case, where it has been studied at three and four loops $[61,62]$.)

The point $v=1$ is not special from the point of view of the amplitude, and we will see that $\mathcal{E}$ has no additional singularities there. The Wilson loop framing (see the next section) can induce logarithmic singularities of the form $\ln |1-v|$ at this point. The point $v=0$ is special because, as shown in section 5, it overlaps with the multi-Regge limit. We will use this correspondence to determing the self-crossing behavior there to high loop orders.

\subsection{Framed Wilson loops}

Before we can discuss the singular behavior of a Wilson loop in the self-crossing limit, starting in section 4, we need to regularize the singularities that it has for any configuration, namely its cusp divergences. A convenient way to do this is to "frame" the Wilson loop [53, 73], as illustrated in figure 5. Instead of considering just the hexagonal Wilson loop, we divide it by two pentagonal Wilson loops and then multiply back by the Wilson loop for a quadrilateral (or box, for short). Each pentagon has three cusps that coincide with three of the six cusps of the hexagon, plus two new ones. Thus dividing by the pentagons removes the cusp singularities of the hexagon, while simultaneously introducing four additional cusps. These cusps are then removed by multiplying by a box which shares two edges and two cusps with each pentagon.

\footnotetext{
${ }^{1}$ The relation between $w$ and $v$ is corrected at $\mathcal{O}(\vec{z})$. However, we can ignore the correction for the MHV case because the amplitudes have no extra singularities as $w \rightarrow v$, so it only leads to power-suppressed terms.
} 


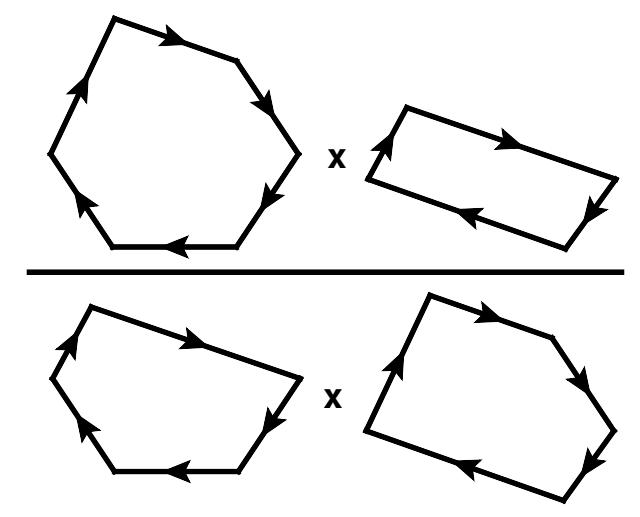

Figure 5. The framing of a hexagonal Wilson loop, by dividing it by two pentagons and multiplying it by a quadrilateral.

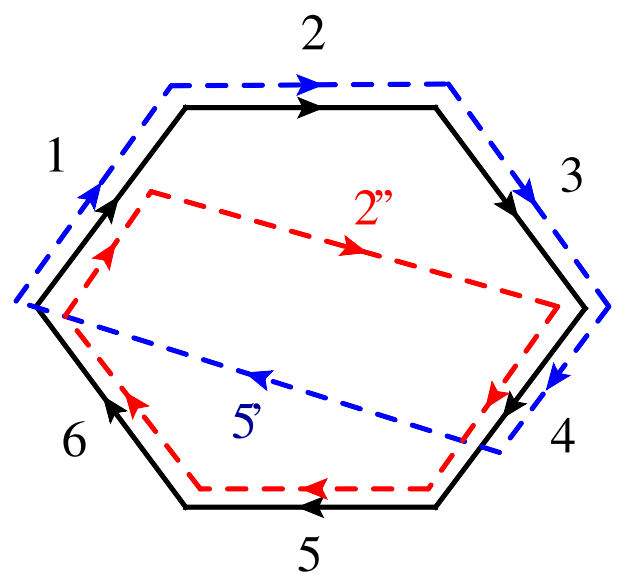

Figure 6. Hexagonal Wilson loop with edges $k_{1}, \ldots, k_{6}$ framed by blue and red dashed pentagons. This framing remains nonsingular in the self-crossing limit.

The first pentagon is defined by selecting one of the corners of the hexagon and creating a new edge connecting it to a light-like separated point on a side furthest away from it, as shown in figure 6 . The second pentagon has the same construction, with all the labels cycled halfway around, by 3 units modulo 6 . It might seem that there are 6 ways to do the framing: 3 pairs of opposite corners to choose, and a twofold ambiguity as to which of the two sides is connected to the first corner. However, taking into account the symmetries of the self-crossing configuration, there are really only three distinct framings. Two of these framings are nonsingular in the self-crossing limit, and we will use one of these. A third framing is singular in the limit, but will still prove useful.

First we describe one of the framings that remains nonsingular in the self-crossing limit. Its Wilson loop will be denoted by $\mathcal{W}^{\text {ns }}$. We use the notation in section 5.4 of ref. [59]. The first pentagon is obtained by removing momenta $k_{4}, k_{5}, k_{6}$ and replacing them with two new momenta $k_{4}^{\prime}, k_{5}^{\prime}$ having the same sum. The vector $k_{4}^{\prime}$ is parallel to $k_{4}$, so we have:

$$
k_{4}+k_{5}+k_{6}=k_{4}^{\prime}+k_{5}^{\prime}, \quad k_{4}^{\prime}=\xi k_{4} .
$$


Demanding $k_{5}^{\prime}$ be light-like fixes $\xi=s_{123} /\left(s_{123}-s_{56}\right)$. The second pentagon is obtained by replacing

$$
k_{1}+k_{2}+k_{3}=k_{1}^{\prime \prime}+k_{2}^{\prime \prime}, \quad k_{1}^{\prime \prime}=\xi^{\prime \prime} k_{1},
$$

and $\xi^{\prime \prime}=s_{123} /\left(s_{123}-s_{23}\right)$. The box then has sides $k_{1}^{\prime \prime}, k_{2}^{\prime \prime}, k_{4}^{\prime}, k_{5}^{\prime}$. This nonsingular framing is illustrated in figure 6 .

Generically, the relation between the framed Wilson loop $\mathcal{W}$ and the remainder function is given by [59]

$$
\mathcal{W}(u, v, w)=\exp \left[R_{6}(u, v, w)+\frac{\gamma_{K}}{8} X(u, v, w)\right]
$$

where

$$
\begin{aligned}
X(u, v, w)= & -\mathrm{Li}_{2}(1-u)-\mathrm{Li}_{2}(1-v)-\mathrm{Li}_{2}(1-w) \\
& -\ln \left(\frac{u v}{w(1-v)}\right) \ln (1-v)-\ln u \ln w+2 \zeta_{2} .
\end{aligned}
$$

We can rewrite this relation in terms of $\mathcal{E}$ using eq. (2.13),

$$
\mathcal{W}(u, v, w)=\mathcal{E}(u, v, w) \exp \left[\frac{\gamma_{K}}{8}(X(u, v, w)+Y(u, v, w))\right] .
$$

In ref. [59] the function $X(u, v, w)$, although correct, was assigned to the framing specified by eqs. (2.25) and (2.26), whereas it should have been to a flipped framing. To say it another way, eqs. (2.25) and (2.26) really correspond to $X(w, v, u)$.

The singular framing is identical to the nonsingular framing, except that the labels of the momenta $k_{i}$ or the dual coordinates $x_{i}$ are lowered cyclically by one unit, $k_{i} \rightarrow k_{i-1}$, $x_{i} \rightarrow x_{i-1}$. This framing is illustrated in figure 7 . Its Wilson loop will be denoted by $\mathcal{W}^{\mathrm{s}}$. It corresponds to letting $u \rightarrow w \rightarrow v \rightarrow u$ in the function $X(w, v, u)$, resulting in the function $X(v, u, w)$. Note that there is a $\ln ^{2}(1-v)$ in $X(u, v, w)$ in eq. (2.28), which becomes a $\ln ^{2}(1-u)$ in $X(v, u, w)$, and is the mathematical origin of additional $\ln ^{2} \delta$ terms that we will find in the self-crossing limit of $X(v, u, w)$ below.

To see physically why this framing is singular, note that in the blue dashed pentagon, leg 6 is adjacent to a light-like leg (call it $4^{\prime}$ ) which runs from the corner between legs 5 and 6 to the middle of leg 3 . But in the self-crossing limit, the point on leg 3 which is light-like separated from the corner between legs 5 and 6 is none other than the self-crossing point, since that lies on both legs 3 and 6 . Therefore the blue pentagon becomes degenerate in this limit; legs $4^{\prime}$ and 6 become collinear. See figure 8. Similarly, the red pentagon degenerates in the self-crossing limit as legs $1^{\prime \prime}$ and 3 become collinear. The box is even more degenerate; it simply runs from the crossing point out and back along leg 3 , and then out and back along leg 6 . For this reason, we will find that the singularly-framed Wilson loop has extra powers of $\ln \delta$ in its perturbative expansion.

In order to convert back and forth between $R_{6}, \mathcal{E}, \mathcal{W}^{\text {ns }}$ and $\mathcal{W}^{\mathrm{s}}$, we also need to record the limiting behavior of $X^{\mathrm{ns}}, X^{\mathrm{s}}$ and $Y$ in the self-crossing limit for both $2 \rightarrow 4$ and $3 \rightarrow 3$ kinematics. For the $2 \rightarrow 4$ self-crossing limit, we first let $u \rightarrow u e^{-2 \pi i}$, so that 


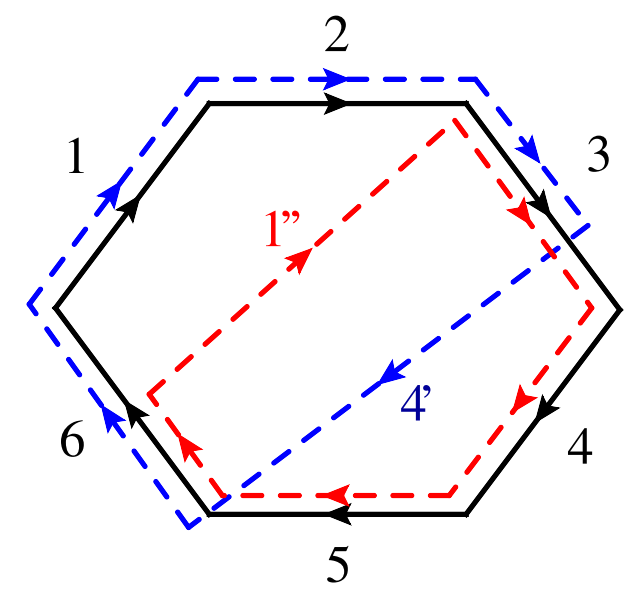

Figure 7. A framing for the hexagonal Wilson loop in which the pentagons and box used to frame the hexagon become singular for self-crossing kinematics.
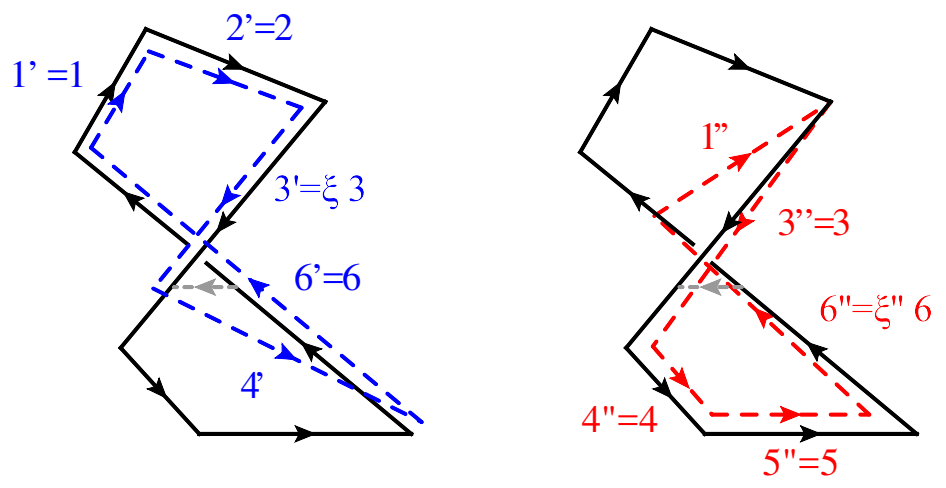

Figure 8. The singular framing in figure 7 near the self-crossing limit. The dashed, gray vector is the regulator $z$. Note that the edges of the framing Wilson loops go straight through the crossing point in this limit. For $z=0$, the edges $k_{6}^{\prime}$ and $k_{4}^{\prime}$ become parallel in the first (blue) framing pentagon, while the edges $k_{1}^{\prime \prime}$ and $k_{3}^{\prime \prime}$ become parallel in the second (red) pentagon. Each framing pentagon degenerates into a box with a parallel segment emerging from it as $z \rightarrow 0$. This behavior induces additional, unwanted dependence on $\delta$. However, it will simplify the $v$ dependence of the singular terms.

$\ln u \rightarrow \ln u-2 \pi i$ and $\operatorname{Li}_{2}(1-u) \rightarrow \operatorname{Li}_{2}(1-u)+2 \pi i \ln (1-u)$. Then we let $u \rightarrow 1-\delta$, $w=v$. We obtain from $X(w, v, u), X(v, u, w)$ and $Y(u, v, w)$ respectively,

$$
\begin{aligned}
X_{2 \rightarrow 4}^{\mathrm{ns}} & =-2 \pi i\left[\ln \delta+\ln \left(\frac{1-v}{v}\right)\right]-2\left(\operatorname{Li}_{2}(1-v)-\zeta_{2}\right)+\ln (1-v)(\ln (1-v)-2 \ln v) \\
X_{2 \rightarrow 4}^{\mathrm{s}} & =\ln ^{2} \delta-2\left(\operatorname{Li}_{2}(1-v)-\zeta_{2}\right)-\ln ^{2} v \\
Y_{2 \rightarrow 4} & =2 \pi i \ln \delta+2 \operatorname{Li}_{2}(1-v)+\ln ^{2} v-2 \pi^{2} \\
& =-X_{2 \rightarrow 4}^{\mathrm{ns}}+2 \pi i \ln \left(\frac{v}{1-v}\right)+\ln ^{2}\left(\frac{v}{1-v}\right)-10 \zeta_{2} .
\end{aligned}
$$

Here we see the $\ln ^{2} \delta$ terms in $X_{2 \rightarrow 4}^{\mathrm{s}}$ whose origin was mentioned earlier. 
To get to $3 \rightarrow 3$ kinematics with $v<0$, following ref. [39] we let $\ln \delta \rightarrow \ln |\delta|-i \pi$, $\ln v \rightarrow \ln |v|-i \pi$, and then complex conjugate the result. We find,

$$
\begin{aligned}
X_{3 \rightarrow 3}^{\mathrm{ns}} & =2 \pi i[\ln |\delta|+L]+2 \operatorname{Li}_{2}(v)+\ln ^{2}(1-v), \\
X_{3 \rightarrow 3}^{\mathrm{s}} & =X_{3 \rightarrow 3}^{\mathrm{ns}}+\ln ^{2}|\delta|-L^{2}, \\
Y_{3 \rightarrow 3} & =-X_{3 \rightarrow 3}^{\mathrm{ns}}+L^{2}-4 \zeta_{2},
\end{aligned}
$$

where

$$
L \equiv \ln \left(1-\frac{1}{v}\right)
$$

Considering eq. (2.35) as well as eq. (2.29), we see that the $3 \rightarrow 3$ self-crossing limit of $\mathcal{E}$ is very closely related to that of the nonsingularly-framed Wilson loop $\mathcal{W}$ :

$$
\begin{aligned}
\mathcal{E}_{3 \rightarrow 3} & =\mathcal{W}_{3 \rightarrow 3}^{\mathrm{ns}} \times \exp \left[-\frac{\gamma_{K}}{8}\left(L^{2}-4 \zeta_{2}\right)\right] \\
& =\mathcal{W}_{3 \rightarrow 3}^{\mathrm{s}} \times \exp \left[-\frac{\gamma_{K}}{8}\left(\ln ^{2}|\delta|-4 \zeta_{2}\right)\right] .
\end{aligned}
$$

\section{$3 \quad$ Explicit results through five loops}

In this section we describe how to extract the $2 \rightarrow 4$ or $3 \rightarrow 3$ self-crossing limit of the MHV amplitude function $\mathcal{E}$, or equivalently the framed hexagonal Wilson loops, from the full remainder function $R_{6}^{(L)}(u, v, w)$, which has been computed for $L=2,3,4[41,47,58,59]$ and recently for $L=5$ [57]. These results include also the nonsingular terms, those having no powers of $\ln \delta$. We will also describe similar results for the NMHV amplitude function $E$, which is entirely nonsingular. Later we will examine the singular terms in the MHV case to even higher loop order by making use of an evolution equation for Wilson loops.

To extract the self-crossing limits, we used properties of hexagon functions [59]. A basis for hexagon functions has been constructed through weight eight [62]. A formula for $R_{6}^{(2)}$ in terms of hexagon functions was presented already in ref. [60], and for $R_{6}^{(3)}$ and $R_{6}^{(4)}$ in ref. [62]. In practice we first found the $2 \rightarrow 4$ limit, and then the $3 \rightarrow 3$ limit by analytic continuation from the $2 \rightarrow 4$ limit. So we will describe that procedure. However, we will only present the $3 \rightarrow 3$ results explicitly, because they are simpler. The $2 \rightarrow 4$ results can be found by reversing the analytic continuation.

\subsection{Analytic continuation from Euclidean to $2 \rightarrow 4$ kinematics}

The analytic continuation for $2 \rightarrow 4$ scattering was described in early studies of the MRK limit [35]. For the $2 \rightarrow 4$ scattering shown in figure 1(a), and given in eq. (A.1), the relevant invariants have the following signs,

$$
s_{12}, s_{45}, s_{36}>0, \quad s_{23}, s_{34}, s_{56}, s_{61}, s_{123}, s_{234}, s_{345}<0 .
$$

We see that $u, v, w>0$. Note that $v$ and $w$ are composed entirely of space-like (negative) invariants. Therefore they do not need to be analytically continued from the Euclidean 
region. In contrast, $u$ is the product of two time-like invariants, divided by two spacelike ones. The $i \varepsilon$ prescription requires $s \rightarrow s e^{-i \pi}$ for each continuation from space-like to time-like, hence to reach the $2 \rightarrow 4$ branch we take

$$
2 \rightarrow 4: \quad u \rightarrow u e^{-2 \pi i}, \quad v \rightarrow v, \quad w \rightarrow w
$$

Next we approach the self-crossing configuration. Like the MRK limit, this requires $u \rightarrow 1$ from below, so we let $u=1-\delta$ with $\delta \ll 1$. In the MRK limit, $v$ and $w$ both approach zero, proportional to $\delta$, but they do not have to be equal. In the self-crossing limit, $v$ and $w$ become equal [28-30]. Thus we need to know how to analytically continue functions under eq. (3.2), followed by the limit $(u, v, w) \rightarrow(1-\delta, v, v)$. Many simplifications occur in this limit. First of all, the quantity $\Delta(u, v, w)=(1-u-v-w)^{2}-4 u v w$ vanishes in this limit, $\Delta(1-\delta, v, v)=-4 \delta v(1-v)+\delta^{2}$. Hexagon functions can be characterized by their "parity", or how they transform under $\Delta \rightarrow-\Delta$. Parity-odd functions are odd under this transformation, and so they vanish like a power of $\delta$ as we approach the line $(1, v, v)$.

Hence we can restrict our attention to hexagon functions $F$ that are even under parity. The coproduct bootstrap for hexagon functions [59] says that we can construct their behavior on the line $(1-\delta, v, v)$ iteratively in the weight, by using the differential equation

$$
\frac{d}{d v} F(1-\delta, v, v)=\frac{F^{v}+F^{w}}{v}-\frac{F^{1-v}+F^{1-w}}{1-v},
$$

where $F^{x}$ denotes the $x$ component of the weight $\{n-1,1\}$ coproduct of the weight $n$ function $F$, evaluated on the same line. In eq. (3.3), we dropped terms involving parity-odd functions, which would arise from coproducts of the form $F^{y_{i}}$, because such contributions vanish on the self-crossing line.

Eq. (3.3) has the same structure as it does on the Euclidean branch, although on that branch it is possible to set $\delta \rightarrow 0$, as there is no singularity as $u \rightarrow 1$ in this case. This limit of the remainder function was given through four loops in refs. [47, 59], and at five loops in ref. [57], as the value on the line $(u, u, 1)$. Since the remainder function is totally symmetric under exchange of its three arguments, this line is equivalent to $(1, v, v)$ up to a relabeling. Just as was found earlier in the Euclidean case, the iterative solution to eq. (3.3) lies in the space of harmonic polylogarithms (HPLs) [74] $H_{\vec{w}}(v)$ with argument $v$ and weight vector $\vec{w}=\left(w_{1}, w_{2}, \ldots, w_{n}\right)$ with all $w_{i} \in\{0,1\}$. The only difference is that on the $2 \rightarrow 4$ and $3 \rightarrow$ 3 self-crossing branches there may be imaginary parts ( $i \pi$ factors) as well as factors of $\ln \delta$.

For example, for the two-loop remainder function $R_{6}^{(2)}$, we can use its $\{3,1\}$ coproducts to write its derivative (3.3) as

$$
\begin{aligned}
v(1-v) \frac{d R_{6}^{(2)}(1-\delta, v, v)}{d v} & =H_{2,1}^{v}-H_{3}^{v}-\left.\frac{1}{2}\left[H_{2}^{u} \ln v+\ln u \ln ^{2} v\right]\right|_{u \rightarrow u e^{-2 \pi i} \rightarrow 1-\delta} \\
& =H_{2,1}^{v}-H_{3}^{v}-i \pi\left[\ln \delta \ln v-\ln ^{2} v\right]
\end{aligned}
$$

where we let $\ln u \rightarrow \ln (1-\delta)-2 \pi i=-2 \pi i$ in the second step. Since $d H_{2}^{u} / d u=(\ln u) /(1-$ $u) \rightarrow-2 \pi i /(1-u)$, we also have that $H_{2}^{u} \rightarrow 2 \pi i \ln (1-u)=2 \pi i \ln \delta$ under this analytic 
continuation. The first two terms of eq. (3.4) match the result found in eq. (7.18) of ref. [59] for the behavior on the Euclidean branch,

$$
\left.v(1-v) \frac{d R_{6}^{(2)}(1, v, v)}{d v}\right|_{\text {Eucl. }}=H_{2,1}^{v}-H_{3}^{v} .
$$

The fact that the $v$ derivative of the remainder function is $1 /[v(1-v)]$ times a transcendental function is just a reflection of the final-entry condition [75]. For general $(u, v, w)$ only six final entries appear:

$$
\left\{\frac{u}{1-u}, \frac{v}{1-v}, \frac{w}{1-w}, y_{u}, y_{v}, y_{w}\right\}
$$

On the line $(1, v, v)$ (on any branch), $u, y_{u}, y_{v}$ and $y_{w}$ are all trivial, and $w=v$, so the set (3.6) collapses to the single final entry $v /(1-v)$, and $d \ln [v /(1-v)] / d v=1 /[v(1-v)]$. So the $v$ derivative of the remainder function must have this prefactor. However, the $v$ derivatives of generic hexagon functions, which are needed in intermediate steps of the iterative construction, will not have this property.

We also need to fix a boundary condition for the integration of eq. (3.3). We do so at the point $v=1$. To obtain the values of the hexagon functions on the $2 \rightarrow 4$ branch at $(1-\delta, 1,1)$, we first obtain them along the Euclidean branch of the line $(u, 1,1)$. On this line, the hexagon functions are also HPLs, with argument $u$, although the parity-odd functions are non-vanishing here. The remainder function is given on this line through four loops in refs. [47, 59], and at five loops in ref. [57]. (See also the ancillary files associated with ref. [62].) For example, the two-loop remainder function is

$$
\begin{gathered}
R_{6}^{(2)}(u, 1,1)=\frac{1}{2}\left[H_{4}^{u}-H_{3,1}^{u}+3 H_{2,1,1}^{u}-\frac{1}{4}\left(H_{2}^{u}\right)^{2}+H_{1}^{u}\left(H_{3}^{u}-2 H_{2,1}^{u}\right)\right. \\
\left.+\frac{1}{2}\left(H_{2}^{u}-\zeta_{2}\right)\left(H_{1}^{u}\right)^{2}-5 \zeta_{4}\right],
\end{gathered}
$$

where $H_{3,1}^{u}=H_{0,0,1,1}(1-u)$, etc. This "standard" Lyndon-basis form of the result has argument $(1-u)$ for all HPLs with trailing 1's in their weight vectors. If there had been a trailing 0 , we could use a shuffle identity to remove it, at the price of extracting a factor of $H_{0}(1-$ $u)=\ln (1-u)$. HPLs with trailing 1's are regular when their argument vanishes. Thus eq. (3.7) is adapted to the point $u=1$, in the sense that it makes manifest that $R_{6}^{(2)}(u, 1,1)$ has no branch cut at $u=1$ (in the Euclidean region), because there is no $\ln (1-u)$.

For the analytic continuation of $u$ around the origin, we need to use HPL identities to rewrite the result in terms of a Lyndon basis for $H_{\vec{w}}(u)$, rather than $H_{\vec{w}}(1-u)$. We obtain,

$$
\begin{aligned}
R_{6}^{(2)}(u, 1,1) & =\frac{1}{4}\left\{\ln u\left[\frac{1}{3}\left[H_{1}(u)\right]^{3}+H_{1}(u)\left[H_{2}(u)+\zeta_{2}\right]+2 H_{3}(u)+2 \zeta_{3}\right]\right. \\
& -6 H_{4}(u)+2 H_{3,1}(u)-2 H_{2,1,1}(u)-\frac{1}{2}\left[H_{2}(u)\right]^{2}-2 H_{1}(u)\left[H_{3}(u)-H_{2,1}(u)\right] \\
- & {\left.\left[H_{1}(u)\right]^{2}\left[H_{2}(u)-\zeta_{2}\right]+\zeta_{2} H_{2}(u)-\frac{15}{4} \zeta_{4}\right\} . }
\end{aligned}
$$


To get to the $2 \rightarrow 4$ branch we just set $\ln u \rightarrow \ln u-2 \pi i$ in eq. (3.8), because the $H_{\vec{w}}(u)$ are all regular at $u=0$. Next we let $u=1-\delta$ and take $\delta \rightarrow 0$, to get

$$
R_{6}^{(2)}(1-\delta, 1,1)=2 \pi i\left[\frac{1}{12} \ln ^{3} \delta+\frac{\zeta_{2}}{2} \ln \delta-\zeta_{3}\right]-\frac{5}{2} \zeta_{4}
$$

Next we need to integrate up eq. (3.3) for the generic hexagon functions, imposing a boundary condition at $(1-\delta, 1,1)$. For the case of $R_{6}^{(2)}$, we integrate eq. (3.4) with the boundary condition (3.9), obtaining

$$
\begin{aligned}
R_{6}^{(2)}(1-\delta, v, v)= & 2 \pi i\left[\frac{1}{12} \ln ^{3} \delta+\left(-\frac{1}{2} H_{2}^{v}-\frac{1}{4} \ln ^{2} v+\frac{\zeta_{2}}{2}\right) \ln \delta-H_{2,1}^{v}+\frac{1}{6} \ln ^{3} v-\zeta_{3}\right] \\
& +H_{4}^{v}-H_{3,1}^{v}+3 H_{2,1,1}^{v}-\ln v\left(H_{3}^{v}-H_{2,1}^{v}\right)-\frac{1}{2}\left(H_{2}^{v}\right)^{2}-\frac{5}{2} \zeta_{4} .
\end{aligned}
$$

The leading $\ln ^{3} \delta$ term in this formula agrees with the result of ref. [28].

We have repeated this exercise for the higher-loop remainder functions $R_{6}^{(3)}, R_{6}^{(4)}$, and $R_{6}^{(5)}$ (as well as for the NMHV coefficient functions $E^{(1)}, E^{(2)}, E^{(3)}$ and $E^{(4)}$ ). Once we have obtained the remainder function in the self-crossing configuration, we can find $\mathcal{W}_{2 \rightarrow 4}^{\text {ns }}$, $\mathcal{W}_{2 \rightarrow 4}^{\mathrm{s}}$ and $\mathcal{E}_{2 \rightarrow 4}$ with the help of eqs. (2.30), (2.31) and (2.32).

\subsection{From $2 \rightarrow 4$ to $3 \rightarrow 3$ kinematics with $v<0$}

To get to $3 \rightarrow 3$ kinematics with $v<0$, following ref. [39] we let $\ln \delta \rightarrow \ln |\delta|-i \pi$, $\ln v \rightarrow \ln |v|-i \pi$, and then complex conjugate the result. To do the analytic continuation around $v=0$, we first rewrite the expressions in terms of a Lyndon basis for $H_{\vec{w}}(v)$. We apply this procedure to the remainder function, and then apply eqs. (2.33), (2.34) and $(2.35)$ to construct the results for the framed Wilson loops and for $\mathcal{E}$. In view of the simple relations (2.37) and (2.38) between them, it's sufficient to give one of these quantities, and $\mathcal{E}$ has the simplest finite parts.

In order to represent the results for $3 \rightarrow 3$ kinematics compactly, we define some compressed notation [47]. We first expand all products of HPLs using the shuffle algebra, in order to linearize the expression in terms of HPLs. The HPL weight vectors $\vec{w}$ consist entirely of 0's and 1's; we encode them as binary numbers, but written as a subscript in decimal. We use a superscript to record the length of the original weight vector. For example,

$$
\begin{aligned}
H_{2}(z) H_{2,1}(z) & =H_{0,1}(z) H_{0,1,1}(z)=6 H_{0,0,1,1,1}(z)+3 H_{0,1,0,1,1}(z)+H_{0,1,1,0,1}(z) \\
& \rightarrow 6 h_{7}^{[5]}+3 h_{11}^{[5]}+h_{13}^{[5]} .
\end{aligned}
$$

Here the suppressed argument of $h$ is $z=1 /(1-v)$. Note also that

$$
h_{1}^{[1]}=-\ln \left(1-\frac{1}{1-v}\right)=\ln \left(1-\frac{1}{v}\right)=L .
$$

In this notation, $X_{3 \rightarrow 3}^{\text {ns }}$ and $Y_{3 \rightarrow 3}$ become

$$
X_{3 \rightarrow 3}^{\mathrm{ns}}=2 \pi i(\ln |\delta|+L)-2 h_{2}^{[2]}-2 \zeta_{2},
$$




$$
Y_{3 \rightarrow 3}=-2 \pi i(\ln |\delta|+L)+2\left(h_{2}^{[2]}+h_{3}^{[2]}\right)-2 \zeta_{2},
$$

and the two-loop remainder function evaluates to

$$
\begin{aligned}
\left.R_{6}^{(2)}\right|_{3 \rightarrow 3}= & 2 \pi i\left[-\frac{1}{12} \ln ^{3}|\delta|+\frac{1}{2}\left(h_{2}^{[2]}+h_{3}^{[2]}+\zeta_{2}\right) \ln |\delta|+\frac{1}{2} h_{5}^{[3]}+h_{6}^{[3]}+h_{7}^{[3]}+\frac{1}{2} \zeta_{2} L+2 \zeta_{3}\right] \\
& +3 \zeta_{2} \ln ^{2}|\delta|+6 \zeta_{2} L \ln |\delta|+2 h_{8}^{[4]}+2 h_{9}^{[4]}+h_{10}^{[4]}+h_{11}^{[4]} \\
& +\zeta_{2}\left(h_{2}^{[2]}+6 h_{3}^{[2]}\right)-\zeta_{3} L+\frac{33}{4} \zeta_{4} .
\end{aligned}
$$

The results for $\mathcal{E}$ in the $3 \rightarrow 3$ self-crossing configuration with $v<0$ through five loops are:

$$
\begin{aligned}
& \mathcal{E}_{3 \rightarrow 3}^{(0)}=1, \\
& \mathcal{E}_{3 \rightarrow 3}^{(1)}=-\frac{1}{2} Y_{3 \rightarrow 3}=\pi i[\ln |\delta|+L]-h_{2}^{[2]}-h_{3}^{[2]}+\zeta_{2}, \\
& \mathcal{E}_{3 \rightarrow 3}^{(2)}=2 \pi i\left[-\frac{1}{12} \ln ^{3}|\delta|+\frac{1}{2} \zeta_{2} \ln |\delta|-\frac{1}{12} L^{3}+\frac{1}{2} \zeta_{2} L+2 \zeta_{3}\right] \\
& +2\left(h_{8}^{[4]}+h_{9}^{[4]}+h_{10}^{[4]}+h_{11}^{[4]}+h_{12}^{[4]}+h_{13}^{[4]}\right) \\
& +3\left(h_{14}^{[4]}+h_{15}^{[4]}\right)+\zeta_{2} h_{2}^{[2]}-\zeta_{3} L+7 \zeta_{4} \\
& \mathcal{E}_{3 \rightarrow 3}^{(3)}=2 \pi i\left[\frac{1}{80} \ln ^{5}|\delta|-\frac{1}{4} \zeta_{3} \ln ^{2}|\delta|+\frac{1}{4} \zeta_{4} \ln |\delta|-\frac{1}{4}\left(4 h_{17}^{[5]}+2 h_{19}^{[5]}+h_{21}^{[5]}+2 h_{25}^{[5]}+h_{27}^{[5]}-6 h_{31}^{[5]}\right.\right. \\
& \left.\left.-\zeta_{2} h_{5}^{[3]}+\zeta_{3} h_{3}^{[2]}+\frac{7}{4} \zeta_{4} L+42 \zeta_{5}-18 \zeta_{2} \zeta_{3}\right)\right] \\
& -\frac{1}{2}\left[24\left(h_{32}^{[6]}+h_{33}^{[6]}\right)+20\left(h_{34}^{[6]}+h_{35}^{[6]}+h_{36}^{[6]}+h_{37}^{[6]}\right)+18\left(h_{38}^{[6]}+h_{39}^{[6]}\right)+20\left(h_{40}^{[6]}+h_{41}^{[6]}\right)\right. \\
& +19\left(h_{42}^{[6]}+h_{43}^{[6]}\right)+18\left(h_{44}^{[6]}+h_{45}^{[6]}+h_{46}^{[6]}+h_{47}^{[6]}\right)+24\left(h_{48}^{[6]}+h_{49}^{[6]}\right) \\
& +22\left(h_{50}^{[6]}+h_{51}^{[6]}+h_{52}^{[6]}+h_{53}^{[6]}\right)+21\left(h_{54}^{[6]}+h_{55}^{[6]}\right) \\
& +24\left(h_{56}^{[6]}+h_{57}^{[6]}+h_{58}^{[6]}+h_{59}^{[6]}+h_{60}^{[6]}+h_{61}^{[6]}\right)+30\left(h_{62}^{[6]}+h_{63}^{[6]}\right) \\
& +\zeta_{2}\left(16 h_{8}^{[4]}+2 h_{9}^{[4]}+11 h_{10}^{[4]}+4 h_{11}^{[4]}+14 h_{12}^{[4]}+7 h_{13}^{[4]}+12 h_{14}^{[4]}+6 h_{15}^{[4]}\right) \\
& +\zeta_{3}\left(2 h_{4}^{[3]}-h_{5}^{[3]}+h_{6}^{[3]}-6 h_{7}^{[3]}\right)+\frac{1}{4} \zeta_{4}\left(81 h_{2}^{[2]}+83 h_{3}^{[2]}\right)-4 \zeta_{5} L \\
& \left.+\frac{3787}{48} \zeta_{6}-\frac{5}{2}\left(\zeta_{3}\right)^{2}\right] \\
& \mathcal{E}_{3 \rightarrow 3}^{(4)}=2 \pi i\left[-\frac{1}{672} \ln ^{7}|\delta|-\frac{1}{80} \zeta_{2} \ln ^{5}|\delta|-\frac{1}{48} \zeta_{3} \ln ^{4}|\delta|-\frac{7}{24} \zeta_{4} \ln ^{3}|\delta|\right. \\
& \left.+\frac{1}{4}\left(4 \zeta_{5}-3 \zeta_{2} \zeta_{3}\right) \ln ^{2}|\delta|-\frac{1}{48}\left(13 \zeta_{6}+48\left(\zeta_{3}\right)^{2}\right) \ln |\delta|\right]+ \text { finite }, \\
& \mathcal{E}_{3 \rightarrow 3}^{(5)}=2 \pi i\left[\frac{1}{6912} \ln ^{9}|\delta|+\frac{1}{336} \zeta_{2} \ln ^{7}|\delta|+\frac{5}{288} \zeta_{3} \ln ^{6}|\delta|+\frac{9}{80} \zeta_{4} \ln ^{5}|\delta|+\frac{1}{24}\left(6 \zeta_{5}+7 \zeta_{2} \zeta_{3}\right) \ln ^{4}|\delta|\right. \\
& +\frac{1}{72}\left(115 \zeta_{6}+48\left(\zeta_{3}\right)^{2}\right) \ln ^{3}|\delta|+\frac{1}{16}\left(-55 \zeta_{7}+68 \zeta_{2} \zeta_{5}+44 \zeta_{3} \zeta_{4}\right) \ln ^{2}|\delta| \\
& \left.+\frac{1}{72}\left(257 \zeta_{8}+810 \zeta_{3} \zeta_{5}+18 \zeta_{2}\left(\zeta_{3}\right)^{2}\right) \ln |\delta|\right]+ \text { finite },
\end{aligned}
$$

where the suppressed argument of the $h_{i}^{[w]}(z)$ is $z=1 /(1-v)$. We also have results for the finite parts of $\mathcal{E}$ at four and five loops, i.e. those terms lacking a power of $\ln |\delta|$. However, these terms are rather lengthy, so we will present them later, for the $v>0$ branch of $3 \rightarrow 3$ kinematics instead (for which they are somewhat more compact); the values for $v<0$ can 
be recovered by analytic continuation. (We give the four- and five-loop finite parts of $\mathcal{E}$ for $v<0$ in the ancillary file accompanying this paper.)

\subsection{A few observations}

Inspection of eqs. (3.16) through (3.21) reveals a number of remarkable features:

1. The $\ln |\delta|$ singularities in $\mathcal{E}_{3 \rightarrow 3}$ are only in the imaginary part. This is not true for the remainder function (see eq. (3.15)), and it is not true for $\mathcal{E}$ in $2 \rightarrow 4$ kinematics. Note that the same statements will be true for both Wilson loops $\mathcal{W}_{3 \rightarrow 3}^{\text {ns }}$ and $\mathcal{W}_{3 \rightarrow 3}^{\mathrm{s}}$, because according to eqs. (2.37) and (2.38), they are related to $\mathcal{E}_{3 \rightarrow 3}$ by real prefactors.

2. The singularities are totally independent of $v$. This statement is also true for the singularly-framed Wilson loop $\mathcal{W}_{3 \rightarrow 3}^{\mathrm{s}}$, but not for $\mathcal{W}_{3 \rightarrow 3}^{\mathrm{ns}}$, since it differs from $\mathcal{E}_{3 \rightarrow 3}$ by a finite, but $v$-dependent exponential factor.

3. In the finite (non-ln $|\delta|$ ) parts, only a limited range of the subscripts $i$ on the functions $h_{i}^{[w]}$ appear, starting at $i=2^{w-1}$. In the imaginary part, only odd values of $i$ appear. In the real part, both even and odd $i$ can appear, but in the non- $\zeta$ terms $i=2 k$ and $i=2 k+1$ always appear with the same coefficients.

The first two properties have their origin in the factorization structure of self-crossed Wilson loops, to be discussed below.

The property that $h_{i}^{[w]}$ never appears for $i<2^{w-1}$ ensures that the binary weight vector for $h_{i}^{[w]}$ always starts with a "1". Then the $v$ derivative of $h_{i}^{[w]}(z)$, with $z=1 /(1-v)$, has a rational prefactor of $d z / d v \times 1 /(1-z)=-1 /[v(1-v)]$. As mentioned earlier, this feature is just the manifestation of the final-entry condition [75] on the remainder function. Inspection of eqs. (3.13) and (3.14) shows that it also holds for $X_{3 \rightarrow 3}^{\text {ns }}$ and $Y_{3 \rightarrow 3}$, so the conversion to $\mathcal{E}$ and the Wilson loop framings do not spoil this property.

Another feature of the explicit results for $\mathcal{W}_{3 \rightarrow 3}^{(L)}$ is that the $h_{i}^{[w]}$ that appear in the imaginary part only have odd values of $i$, while in the real part there is no such restriction. An odd value of $i$, or a final value of " 1 " in the binary representation, corresponds to a statement that the branch cuts in $\mathcal{W}_{3 \rightarrow 3}^{(L)}$ are only in $1-z=-v /(1-v)$. This property allows for a branch cut at $v=0$ (which definitely occurs) but forbids it at $v=\infty$. Recall that $v=\infty$ is the position of the multi-particle pole as $s_{234} \rightarrow 0$ inside $3 \rightarrow 3$ kinematics, and that helicity selection rules forbid such a pole in the MHV case. Although there is no pole, there certainly can be a branch cut in this channel. It is interesting that the branch cut behavior in $v$ is only found in the real part, not the imaginary part.

The leading $\ln ^{5}|\delta|$ term in the remainder function $R_{6}^{(3)}$, was predicted in ref. [30] to be $R_{6}^{(3)} \sim \mp(7 \pi i / 240) \ln ^{5}|\delta|$. At this leading order in $\ln |\delta|$, the behavior of $R_{6}^{(3)}, \mathcal{E}^{(3)}$ and the nonsingularly-framed Wilson loop $\mathcal{W}_{3 \rightarrow 3}^{\text {ns }}$ are identical; for example, the cross-terms from exponentiation can produce at most a term of the form $\pi^{2} \ln ^{4}|\delta|$. Eq. (3.19) disagrees with the prediction of ref. [30] by a factor of $\pm 6 / 7$. What could cause the discrepancy? In ref. [30], the same dimensional regulator $\epsilon$ was used to regulate the self-crossing singularity as the cusp singularity. This may have led to difficulties in extracting the dependence 
on the self-crossing separation alone. Here we have separated the self-crossing and cusp singularities cleanly; the nonsingular framing removes the cusp singularities completely, at least at this order in $\ln |\delta|$. Also, we use a dual conformal measure $\delta$ of the self-crossing separation throughout the calculation.

\subsection{From $v<0$ to $v>1$}

Formulas (3.16) to (3.19) describe the MHV amplitude in $3 \rightarrow 3$ kinematics with $v<0$, or $s_{234}>0$. In this subsection we describe how to analytically continue them to the other branch of $3 \rightarrow 3$ kinematics, where $s_{234}<0$ and $v>1$. From eq. (2.22) we see that the sign of $\delta$ does not change in passing between the two branches, because $s_{36}$ remains negative. We just need to analytically continue $v$ around $v=\infty$. Letting $s_{234} \rightarrow s_{234}+i \varepsilon$ in eq. (2.7), we find the sign of the $i \pi$ term in the continuation:

$$
\ln \left(\frac{1}{1-v}\right) \rightarrow \ln \left(\frac{1}{v-1}\right)+i \pi
$$

In order to carry out this analytic continuation of eqs. (3.16)-(3.19), it is simplest to return to the Lyndon basis for HPLs $H_{\vec{w}}(z)$ with argument $z=1 /(1-v)$. That's because the point $v=\infty$ around which we are continuing is at $z=0$, and all HPLs with trailing 1 's in their weight vectors are regular at this point. In the Lyndon basis, the only function that is not regular is

$$
H_{0}(z)=\ln \left(\frac{1}{1-v}\right)
$$

which is to be replaced using eq. (3.22). Then we rewrite the result in a linearized basis, for compactness, but using a different argument for the $h$ functions, $\hat{z}=1 / v$.

The results for the $3 \rightarrow 3$ self-crossing configuration with $v>1$ through three loops are:

$$
\begin{aligned}
& \mathcal{E}_{3 \rightarrow 3}^{(0)}(v>1)=1, \\
& \mathcal{E}_{3 \rightarrow 3}^{(1)}(v>1)=\pi i \ln |\delta|+h_{2}^{[2]}+\zeta_{2}, \\
& \mathcal{E}_{3 \rightarrow 3}^{(2)}(v>1)=2 \pi i\left[-\frac{1}{12} \ln ^{3}|\delta|+\frac{1}{2} \zeta_{2} \ln |\delta|-h_{4}^{[3]}+2 \zeta_{3}\right] \\
& -2 h_{8}^{[4]}-h_{14}^{[4]}+\zeta_{2}\left(5 h_{2}^{[2]}-h_{3}^{[2]}\right)+\zeta_{3} h_{1}^{[1]}+7 \zeta_{4}, \\
& \mathcal{E}_{3 \rightarrow 3}^{(3)}(v>1)=2 \pi i\left\{\frac{1}{80} \ln ^{5}|\delta|-\frac{1}{4} \zeta_{3} \ln ^{2}|\delta|+\frac{1}{4} \zeta_{4} \ln |\delta|+\frac{1}{2}\left[12 h_{16}^{[5]}+2 h_{18}^{[5]}+2 h_{20}^{[5]}+h_{22}^{[5]}\right.\right. \\
& \left.\left.+h_{26}^{[5]}+2 h_{28}^{[5]}+\zeta_{2}\left(-4 h_{4}^{[3]}+h_{5}^{[3]}+h_{6}^{[3]}\right)+\zeta_{3} h_{2}^{[2]}-21 \zeta_{5}+9 \zeta_{2} \zeta_{3}\right]\right\} \\
& +\frac{1}{2}\left[24 h_{32}^{[6]}+4 h_{34}^{[6]}+4 h_{36}^{[6]}+2 h_{38}^{[6]}+4 h_{40}^{[6]}+3 h_{42}^{[6]}+2 h_{44}^{[6]}+h_{46}^{[6]}+2 h_{50}^{[6]}\right. \\
& +2 h_{52}^{[6]}+h_{54}^{[6]}+4 h_{56}^{[6]}+h_{58}^{[6]}+6 h_{62}^{[6]} \\
& +\zeta_{2}\left(-56 h_{8}^{[4]}+2 h_{9}^{[4]}-7 h_{10}^{[4]}+h_{11}^{[4]}+2 h_{12}^{[4]}+h_{13}^{[4]}-9 h_{14}^{[4]}+6 h_{15}^{[4]}\right) \\
& +\zeta_{3}\left(2 h_{4}^{[3]}+3 h_{5}^{[3]}+h_{6}^{[3]}-4 h_{7}^{[3]}\right)-\frac{1}{4} \zeta_{4}\left(39 h_{2}^{[2]}+62 h_{3}^{[2]}\right)
\end{aligned}
$$




$$
\left.-\left(4 \zeta_{5}+6 \zeta_{2} \zeta_{3}\right) h_{1}^{[1]}-\frac{3787}{48} \zeta_{6}+\frac{5}{2}\left(\zeta_{3}\right)^{2}\right]
$$

where the suppressed argument of the $h_{i}^{[w]}(\hat{z})$ is now $\hat{z}=1 / v$. The corresponding results for four and five loops are given in eqs. (C.1) and (C.2) in appendix C.

The singular terms for $v>1$ in eqs. (3.24) through (3.27), and in eqs. (C.1) and (C.2), are identical to those for the $v<0$ branch. The finite terms are even simpler, thanks to the choice of $\hat{z}$ argument. The binary vectors for $i$ in $h_{i}^{[w]}$ still start with a "1" as a consequence of the final entry condition, because $d \hat{z} / d v \times 1 /(1-\hat{z})=1 /[v(1-v)]$. However, now there are only even values of $i$ for the non- $\zeta$ parts of both the imaginary and the real parts of $\mathcal{E}_{3 \rightarrow 3}(v>1)$. An odd value of $i$ would correspond to a branch cut at $v=1$; however, appendix A shows that this is an unremarkable scattering configuration, and the amplitude is totally smooth there.

\section{$3.5 v \rightarrow \infty$ and $v \rightarrow 1$ limits}

From the formulas (3.24) to (3.27), (C.1) and (C.2), it is straightforward to take the limit $v \rightarrow \infty$, which just amounts to setting all the $h_{i}^{[w]}$ to zero, since they all vanish at $\hat{z}=1 / v=0$. Equivalently, one can set all the $h_{i}^{[w]}$ to zero in the previous formulas for $v<0$, since $z=1 /(1-v)=0$ in this limit too. Through five loops, one gets the same result as one approaches $v=\infty$ from either the positive or the negative side, and the results contain no $\ln v$ divergences:

$$
\begin{aligned}
& \mathcal{E}_{3 \rightarrow 3}^{(0)}(v=\infty)=1, \\
& \mathcal{E}_{3 \rightarrow 3}^{(1)}(v=\infty)=\pi i \ln |\delta|+\zeta_{2}, \\
& \mathcal{E}_{3 \rightarrow 3}^{(2)}(v=\infty)=2 \pi i\left[-\frac{1}{12} \ln ^{3}|\delta|+\frac{1}{2} \zeta_{2} \ln |\delta|+2 \zeta_{3}\right]+7 \zeta_{4}, \\
& \mathcal{E}_{3 \rightarrow 3}^{(3)}(v=\infty)=2 \pi i\left[\frac{1}{80} \ln ^{5}|\delta|-\frac{1}{4} \zeta_{3} \ln ^{2}|\delta|+\frac{1}{4} \zeta_{4} \ln |\delta|-\frac{21}{2} \zeta_{5}+\frac{9}{2} \zeta_{2} \zeta_{3}\right] \\
& -\frac{3787}{96} \zeta_{6}+\frac{5}{4}\left(\zeta_{3}\right)^{2} \\
& \mathcal{E}_{3 \rightarrow 3}^{(4)}(v=\infty)=2 \pi i\left[-\frac{1}{672} \ln ^{7}|\delta|-\frac{1}{80} \zeta_{2} \ln ^{5}|\delta|-\frac{1}{48} \zeta_{3} \ln ^{4}|\delta|-\frac{7}{24} \zeta_{4} \ln ^{3}|\delta|\right. \\
& +\frac{1}{4}\left(4 \zeta_{5}-3 \zeta_{2} \zeta_{3}\right) \ln ^{2}|\delta|-\frac{1}{48}\left(13 \zeta_{6}+48\left(\zeta_{3}\right)^{2}\right) \ln |\delta| \\
& \left.+\frac{1141}{16} \zeta_{7}-\frac{119}{4} \zeta_{2} \zeta_{5}-\frac{17}{4} \zeta_{3} \zeta_{4}\right] \\
& +\frac{5}{4} \zeta_{5,3}+\frac{56911}{144} \zeta_{8}+18 \zeta_{2}\left(\zeta_{3}\right)^{2}-\frac{63}{4} \zeta_{3} \zeta_{5}, \\
& \mathcal{E}_{3 \rightarrow 3}^{(5)}(v=\infty)=2 \pi i\left[\frac{1}{6912} \ln ^{9}|\delta|+\frac{1}{336} \zeta_{2} \ln ^{7}|\delta|+\frac{5}{288} \zeta_{3} \ln ^{6}|\delta|+\frac{9}{80} \zeta_{4} \ln ^{5}|\delta|\right. \\
& +\frac{1}{24}\left(6 \zeta_{5}+7 \zeta_{2} \zeta_{3}\right) \ln ^{4}|\delta|+\frac{1}{72}\left(115 \zeta_{6}+48\left(\zeta_{3}\right)^{2}\right) \ln ^{3}|\delta| \\
& +\frac{1}{16}\left(-55 \zeta_{7}+68 \zeta_{2} \zeta_{5}+44 \zeta_{3} \zeta_{4}\right) \ln ^{2}|\delta|
\end{aligned}
$$




$$
\begin{aligned}
& +\frac{1}{72}\left(257 \zeta_{8}+18 \zeta_{2}\left(\zeta_{3}\right)^{2}+810 \zeta_{3} \zeta_{5}\right) \ln |\delta| \\
& \left.-\frac{40369}{64} \zeta_{9}+\frac{7645}{32} \zeta_{2} \zeta_{7}+\frac{3119}{64} \zeta_{3} \zeta_{6}+\frac{2295}{32} \zeta_{4} \zeta_{5}-\frac{15}{4}\left(\zeta_{3}\right)^{3}\right] \\
& -\frac{177}{28} \zeta_{7,3}+\frac{1217}{40} \zeta_{2} \zeta_{5,3}-\frac{2668732849}{537600} \zeta_{10}-\frac{2659}{32} \zeta_{4}\left(\zeta_{3}\right)^{2} \\
& -\frac{3091}{8} \zeta_{2} \zeta_{3} \zeta_{5}+\frac{9179}{64} \zeta_{3} \zeta_{7}+\frac{20553}{224}\left(\zeta_{5}\right)^{2} .
\end{aligned}
$$

Multiple zeta values (MZV's) begin to appear at four loops:

$$
\zeta_{5,3}=\sum_{n_{1}>n_{2}>0} \frac{1}{n_{1}^{5} n_{2}^{3}}=0.0377076729848 \ldots, \quad \zeta_{7,3}=\sum_{n_{1}>n_{2}>0} \frac{1}{n_{1}^{7} n_{2}^{3}}=0.0084196685030 \ldots
$$

Even though there is a branch cut at $v=\infty$, the residue vanishes there, and we get the same limiting behavior as $v$ becomes large for either sign of $v$. The nonsingularly framed Wilson loop $\mathcal{W}^{\text {ns }}$ has a very similar behavior to $\mathcal{E}$ in this limit, because $L^{2} \rightarrow 0$ as $v \rightarrow \infty$, so the exponential factor in eq. (2.37) simply approaches $\exp \left[\frac{1}{2} \zeta_{2} \gamma_{K}\right]$.

The limit of $\mathcal{E}_{3 \rightarrow 3}(v)$ as $v \rightarrow 1$ from above is also smooth in $v$. In this case the $h_{i}^{[w]}$ have to be evaluated at $\hat{z}=1 / v=1$, where they are given by multiple zeta values. Since the $\ln |\delta|$ terms are exactly the same as for other $3 \rightarrow 3$ values of $v$, here we just present the finite $($ non- $\ln |\delta|)$ terms:

$$
\begin{aligned}
\mathcal{E}_{3 \rightarrow 3}^{(0), \text { fin }}\left(v \rightarrow 1^{+}\right)= & 1 \\
\mathcal{E}_{3 \rightarrow 3}^{(1), \text { fin }}\left(v \rightarrow 1^{+}\right)= & 0 \\
\mathcal{E}_{3 \rightarrow 3}^{(2), \text { fin }}\left(v \rightarrow 1^{+}\right)= & 2 \pi i \zeta_{3}-\frac{5}{2} \zeta_{4} \\
\mathcal{E}_{3 \rightarrow 3}^{(3), \text { fin }}\left(v \rightarrow 1^{+}\right)= & 2 \pi i\left[-4 \zeta_{5}+2 \zeta_{2} \zeta_{3}\right]+\frac{35}{24} \zeta_{6}+\left(\zeta_{3}\right)^{2}, \\
\mathcal{E}_{3 \rightarrow 3}^{(4), \text { fin }}\left(v \rightarrow 1^{+}\right)= & 2 \pi i\left[\frac{39}{2} \zeta_{7}-\frac{19}{2} \zeta_{2} \zeta_{5}+\frac{5}{4} \zeta_{3} \zeta_{4}\right]+\frac{3}{2} \zeta_{5,3}-\frac{77}{48} \zeta_{8}-\frac{5}{2} \zeta_{3} \zeta_{5}+\frac{15}{2} \zeta_{2}\left(\zeta_{3}\right)^{2}, \\
\mathcal{E}_{3 \rightarrow 3}^{(5), \text { fin }}\left(v \rightarrow 1^{+}\right)= & 2 \pi i\left[-\frac{857}{8} \zeta_{9}+\frac{205}{4} \zeta_{2} \zeta_{7}-\frac{299}{48} \zeta_{3} \zeta_{6}-\frac{45}{4} \zeta_{4} \zeta_{5}-\frac{1}{2}\left(\zeta_{3}\right)^{3}\right] \\
& -6 \zeta_{7,3}+\frac{15}{2} \zeta_{2} \zeta_{5,3}-\frac{3961}{96} \zeta_{10}+\frac{29}{2} \zeta_{4}\left(\zeta_{3}\right)^{2}-60 \zeta_{2} \zeta_{3} \zeta_{5} \\
& -\frac{63}{2} \zeta_{3} \zeta_{7}-\frac{111}{8}\left(\zeta_{5}\right)^{2} .
\end{aligned}
$$

For what it's worth, the rational numbers multiplying the $\zeta$ values in these equations seem to be quite a bit simpler than in most other limits of these functions.

One can also obtain the results in the limit of $2 \rightarrow 4$ kinematics as $v \rightarrow 1^{-}$by analytic continuation. In general, one should let $\ln |\delta| \rightarrow \ln \delta-i \pi$ and $\ln (v-1) \rightarrow \ln (1-v)+i \pi$, and then complex conjugate the resulting expression. For $\mathcal{E}$ the step $\ln (v-1) \rightarrow \ln (1-v)+i \pi$ can be omitted since there is no $\ln (v-1)$ singularity. These results obtained in this way agree with those obtained by taking the limit $v \rightarrow 1^{-}$directly from expressions (not shown) for $\mathcal{E}_{2 \rightarrow 4}(v)$. 
In contrast to the smooth behavior of $\mathcal{E}$ as $v \rightarrow 1$, the limit of the nonsingularly-framed Wilson loop $\mathcal{W}_{3 \rightarrow 3}^{\text {ns }}(v)$ is divergent as $v \rightarrow 1^{+}$; the divergence is simply due to the factor in eq. (2.37) of $\exp \left[-\gamma_{K} L^{2} / 8\right] \approx \exp \left[-\gamma_{K} \ln ^{2}(v-1) / 8\right]$. If one approaches $v=1$ from the $2 \rightarrow 4$ side, i.e. $v \rightarrow 1^{-}$, then there are additional phases in the divergence due to the analytic continuation $\ln (v-1) \rightarrow \ln (1-v)+i \pi$.

\section{Evolving framed self-crossing Wilson loops}

\subsection{A simple evolution equation}

Framing the hexagon Wilson loop removes its cusp singularities and makes $\mathcal{W}^{\text {ns }}$ an ultraviolet (UV) finite, dual conformal invariant function $\mathcal{W}^{\text {ns }}(u, v, w)$. Denote the non-framed hexagonal Wilson loop by $W_{\text {hex }} \equiv W_{6}$, and denote the combination of two pentagon and one box Wilson loops used to do the non-singular framing by $W_{\mathrm{f}}$ (the subscript "f" refers to the framing), so that

$$
\mathcal{W}^{\mathrm{ns}}=W_{\mathrm{hex}} \times W_{\mathrm{f}}
$$

Note that because of UV divergences, neither $W_{\text {hex }}$ nor $W_{\mathrm{f}}$ are purely functions of the cross ratios $u, v, w$, but their product in eq. (4.1) is.

Consider the self-crossing limit, in which edges $k_{3}$ and $k_{6}$ cross (see figure 2 ). In terms of dual conformal variables the limit is $(u, v, w) \rightarrow(1, v, v)$. We approach the limit in the following way: first move onto the plane $w=v \cdot \mathcal{W}^{\text {ns }}$ does not acquire any divergences in this step. Then approach the line $u=1$ by taking $u=1-\delta$ :

$$
\mathcal{W}^{\mathrm{ns}}(u, v, w) \rightarrow \mathcal{W}^{\mathrm{ns}}(u, v, v) \rightarrow \mathcal{W}^{\mathrm{ns}}(1-\delta, v, v) .
$$

We choose the non-singular framing $W_{\mathrm{f}}$ because the new light-like lines in the pentagons and the box do not go near the self-crossing point. Thus the framing Wilson loops do not acquire any additional singular behavior in the self-crossing limit (in contrast to the singular framing defining $\mathcal{W}^{\mathrm{s}}$ ).

Moreover, since the job of $W_{\mathrm{f}}$ is to remove cusp divergences, any divergences in $\delta$ for $\mathcal{W}^{\text {ns }}$ are purely due to $W_{\text {hex }}$. We expect these divergences to be governed by an evolution equation of the form

$$
\frac{d}{d \ln \delta} \mathcal{W}^{\mathrm{ns}}(1-\delta, v, v)=\mathcal{F}(\delta, v)
$$

for some function $\mathcal{F}$.

To find an equation of the form (4.3) we follow the approach of Korchemskaya and Korchemsky [32], who studied the crossing of two infinite Wilson lines, not necessarily light-like. Consider two auxiliary Wilson loops $W_{1}$ and $W_{2}$, depicted in figure 9 . The only difference between $W_{1}$ and $W_{\text {hex }}$ is that $W_{1}$ is evaluated strictly in the self-crossing kinematics; hence $W_{1}$ is formally infinite and must be renormalized. $W_{\text {hex }}$ is finite for $\vec{z} \neq 0$ and is equal to $W_{1}$ for $\vec{z}=0$. The second Wilson loop in figure $9, W_{2}$, is the same as $W_{1}$, except that the routing of the lines at the self-crossing point is exchanged so that the contour forms two boxes instead of a hexagon. It is also evaluated with the two vertices on top of each other, after renormalization. The renormalized quantities $W_{1}^{r}$ and $W_{2}^{r}$ are finite, 


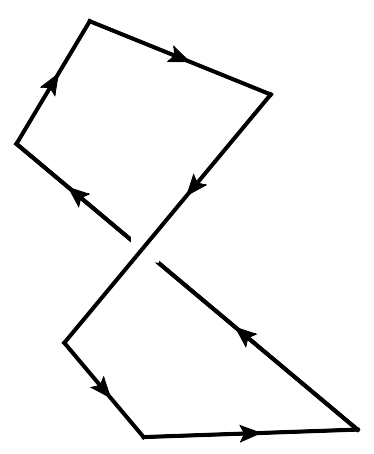

$W_{1}$

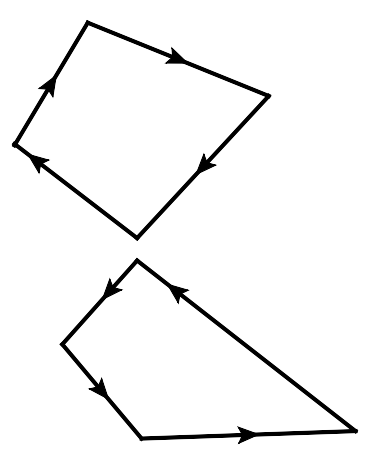

$W_{2}$

Figure 9. Wilson loops $W_{1}$ and $W_{2}$ mix under renormalization.

non-trivial functions of the renormalization scale $\mu$. Under a change of renormalization scale, they mix with each other [33, 34]. The functional dependence of $W_{1}^{r}$ on $\mu^{2}$ should be the same as the functional dependence of $W_{\text {hex }}$ on the inverse separation $1 / \vec{z}^{2}$. We will find a renormalization group (RG) equation for $W_{1}^{r}\left(\mu^{2}\right)$ and then set $\mu^{2}=1 / \vec{z}^{2}$ in order to obtain an RG equation for $W_{\text {hex }}$. This will be used, in turn, to obtain a differential equation for $\mathcal{W}^{\text {ns }}$. Rewriting $\vec{z}^{2}$ in terms of $\delta$ will give an equation of the form (4.3).

The RG equations mixing the renormalized Wilson loops $W_{1}^{r}$ and $W_{2}^{r}$ for a general theory are $[32-34]$

$$
\left(\mu \frac{\partial}{\partial \mu}+\beta(g) \frac{\partial}{\partial g}\right) W_{i}^{r}=-\Gamma^{i j}(\gamma, g) W_{j}^{r}-\sum_{m} \Gamma_{\text {cusp }}\left(\gamma_{m}, g\right) W_{i}^{r} .
$$

Here $\gamma$ is the crossing angle, $m$ labels the cusps of $W_{1}^{r}$, and $\gamma_{m}$ are the corresponding cusp angles. The cusp anomalous dimension for finite cusp angle $\gamma_{m}$ is denoted by $\Gamma_{\text {cusp }}\left(\gamma_{m}, g\right)$, and $\Gamma^{i j}(\gamma, g)$ is the cross anomalous dimension matrix.

Eq. (4.4) holds for massive Wilson lines, for which all cusp angles and the angle at the self-crossing point are finite. Below we give a modified relation for the massless case. In the subsequent equations we imagine keeping the Wilson lines massive and taking the massless limit at the end. Finally, in $\mathcal{N}=4$ SYM, the beta function vanishes and eq. (4.4) simplifies to

$$
\mu \frac{\partial}{\partial \mu} W_{i}^{r}=-\Gamma^{i j}(\gamma, g) W_{j}^{r}-\sum_{m} \Gamma_{\operatorname{cusp}}\left(\gamma_{m}, g\right) W_{i}^{r}
$$

Let $\mathcal{W}_{i}^{r} \equiv W_{i}^{r} \times W_{\mathrm{f}}$. As discussed above, the (renormalized) framing function $W_{\mathrm{f}}$ removes all six cusp divergences in the massive case where $\gamma$ is finite. This is true even for $\mathcal{W}_{2}^{r}$, even though $W_{\mathrm{f}}$ is the framing function for the hexagon and $W_{2}^{r}$ is the renormalized Wilson loops for two boxes, because the six cusp angles match. (In our terminology, we do not count the two cusps in $\mathcal{W}_{2}^{r}$ at the self-crossing point as cusps; we handle them separately.) In the massless limit, where $\gamma_{m}$ and $\gamma$ go to infinity, things are more subtle. 
Taking into account the removal of the cusp divergences when passing from $W_{i}^{r}$ to $\mathcal{W}_{i}^{r}$, we obtain

$$
\mu \frac{\partial}{\partial \mu} \mathcal{W}_{i}^{r}=-\Gamma^{i j}(\gamma, g) \mathcal{W}_{j}^{r}
$$

The components $\Gamma^{i j}(\gamma, g)$ in the large $N_{c}$ limit of QCD and in the limit of large $\gamma$ can be found in ref. [32]. An important point is that, after inserting an appropriate factor of $N_{c}$ into the normalization of $\mathcal{W}_{2}^{r}$, they take an upper triangular form in the large $N_{c}$ limit [28]. The reason is that for a gluon to contribute to the evolution of $\mathcal{W}_{1}^{r}$ it should be exchanged between the two self-crossing lines, which produces an extra factor of $N_{c}$, allowing $\mathcal{W}_{1}^{r}$ to mix into $\mathcal{W}_{2}^{r}$; whereas for a gluon to cause evolution of $\mathcal{W}_{2}^{r}$ into $\mathcal{W}_{1}^{r}$, its exchange between the two boxes in figure 9 is color suppressed, by a factor of $1 / N_{c}$. Since $\Gamma^{11}=\Gamma^{21}=0$ at large $N_{c}$, the evolution of both $\mathcal{W}_{1}^{r}$ and $\mathcal{W}_{2}^{r}$ is governed by $\mathcal{W}_{2}^{r}$, through the matrix elements $\Gamma^{12}$ and $\Gamma^{22}$. Furthermore, $\Gamma^{22}$ is proportional to the cusp anomalous dimension.

\subsection{The large $\gamma$ limit}

In the limit of large $\gamma$, ref. [32] finds that $\Gamma^{12}$ behaves like a constant times $i \pi$ in QCD, while the leading behavior of the cusp anomalous dimension is proportional to $\gamma \times \gamma_{K}$, where $\gamma_{K}$ is the light-like cusp anomalous dimension. We assume a similar form for the matrix elements holds here (see also the discussion in ref. [28]),

$$
\begin{aligned}
& \Gamma^{12} \rightarrow-i \pi \Gamma_{1}(a), \\
& \Gamma^{22} \rightarrow \gamma \frac{\gamma_{K}(a)}{2},
\end{aligned}
$$

as $\gamma \rightarrow \infty$, where we converted to our normalization of the cusp anomalous dimension and perturbative expansion parameter $a=g_{\mathrm{YM}}^{2} N_{c} /\left(8 \pi^{2}\right)$. Inserting these values into eq. (4.6), we find

$$
\begin{aligned}
& \mu \frac{\partial}{\partial \mu} \mathcal{W}_{1}^{r}=i \pi \Gamma_{1}(a) \mathcal{W}_{2}^{r}, \\
& \mu \frac{\partial}{\partial \mu} \mathcal{W}_{2}^{r}=-\gamma \frac{\gamma_{K}(a)}{2} \mathcal{W}_{2}^{r} .
\end{aligned}
$$

In the massless limit $\gamma \rightarrow \infty$, eq. (4.10) is not defined. We can attempt to get into this limit using the method of refs. [28, 76]. Taking legs 3 and 6 to be massive initially, with masses $k_{3}^{2}$ and $k_{6}^{2}$, we write eq. (4.10) as

$$
\mu \frac{\partial}{\partial \mu} \ln \mathcal{W}_{2}^{r} \stackrel{?}{=}-\gamma \frac{\gamma_{K}(a)}{2}
$$

where

$$
\gamma=\ln \left(\frac{s_{36}}{\sqrt{k_{3}^{2}} \sqrt{k_{6}^{2}}}\right) .
$$

Now differentiate eq. (4.11) with respect to $s_{36}$, and integrate back up to obtain

$$
\mu \frac{\partial}{\partial \mu} \mathcal{W}_{2}^{r} \stackrel{?}{=}\left(-\frac{\gamma_{K}(a)}{2} \log \left(\mu^{2} s_{36}\right)-\bar{\Gamma}(a)\right) \mathcal{W}_{2}^{r},
$$


where $\bar{\Gamma}(a)$ is an integration constant. Notice the appearance of the non-dual-conformal quantity $s_{36}$. This signals a problem with the large $\gamma$ limit for eq. (4.10). If we ignore this problem, we can trade $\mu^{2}$ for $1 / \vec{z}^{2} \rightarrow\left[\delta s_{36} x y(1-x)(1-y)\right]^{-1}$ by the prescription described in eq. (2.22). Dropping the non-dual-conformal factor $x y(1-x)(1-y)$, eq. (4.13) becomes, in $3 \rightarrow 3$ kinematics,

$$
\frac{d}{d \ln |\delta|} \ln \mathcal{W}_{2} \stackrel{?}{=}-\frac{\gamma_{K}(a)}{4} \ln |\delta|+\frac{\bar{\Gamma}}{2}
$$

which integrates to

$$
\mathcal{W}_{2}(\delta, v) \stackrel{?}{=} f(v) \exp \left[-\frac{\gamma_{K}(a)}{8} \ln ^{2}|\delta|+\frac{\bar{\Gamma}(a)}{2} \ln |\delta|\right],
$$

for some function $f(v)$ of $v$ alone. However, we will see below that such a solution is inconsistent with explicit results.

More important for our purposes is eq. (4.9), because it contains no explicit $\gamma$, so we expect its large $\gamma$ limit to be reliable. Again trading $\mu^{2}$ for $1 / \delta$, eq. (4.9) becomes

$$
\frac{1}{2 \pi i} \frac{d}{d \ln \delta} \mathcal{W}^{\mathrm{ns}}(1-\delta, v, v)=-\frac{1}{4} \Gamma_{1}(a) \mathcal{W}_{2}(\delta, v) .
$$

We have changed notation $\mathcal{W}_{2}^{r} \rightarrow \mathcal{W}_{2}, \mathcal{W}_{1}^{r} \rightarrow \mathcal{W}^{\text {ns }}$, to emphasize that, here and below, the renormalization scale $\mu$ has been exchanged for $\delta$ using eq. (2.22), so that we now deal with finite functions (for non-zero $\delta$ ) of the dual conformal variable $v$.

\subsection{The framed double box}

In order to separate the various functional dependences of $\mathcal{W}^{\text {ns }}$, it will be useful to rewrite $\mathcal{W}_{2}$ as $\mathcal{W}_{2}=W_{2} \times W_{\mathrm{f}}=W_{2} \times W_{\mathrm{f}}^{\mathrm{s}} \times\left(W_{\mathrm{f}} / W_{\mathrm{f}}^{\mathrm{s}}\right)$, where $W_{\mathrm{f}}^{\mathrm{s}}$ is a new framing function, corresponding to the singular framing in figure 7 , for which we defined $\mathcal{W}^{\mathrm{s}}=W_{\text {hex }} \times W_{\mathrm{f}}^{\mathrm{s}}$ back in section 2.4. (The superscript "s" reminds us that this framing function is singular in the self-crossing kinematics.)

The reason for rewriting things in this way is that the framing function $W_{\mathrm{f}}$ involves pentagons that straddle both sides of the self-crossing geometry. Thus they have sensitivity to the global geometry of the hexagon, and can carry non-trivial $v$ dependence (although $W_{\mathrm{f}}$ is not dual conformally invariant separately from $\left.W_{\text {hex }}\right)$. On the other hand, the framing corresponding to $W_{\mathrm{f}}^{\mathrm{s}}$ is so degenerate in the self-crossing limit, consisting essentially only of boxes on one side of the crossing point or the other, that it cannot depend on $v$ (see figure 8 ). For $3 \rightarrow 3$ kinematics, using the exact results in $[66,67]$ for four- and five-sided Wilson loops, or equivalently by comparing eqs. (2.37) and (2.38) for the different hexagon framings, we can relate the two framing functions:

$$
\frac{W_{\mathrm{f}}}{W_{\mathrm{f}}^{\mathrm{s}}}=\frac{\mathcal{W}_{2}}{\mathcal{W}_{2}^{\mathrm{s}}}=\exp \left[-\frac{\gamma_{K}(a)}{8}\left(\ln ^{2}|\delta|-L^{2}\right)\right]
$$

where $L=\ln (1-1 / v)$.

The evolution equation (4.16) becomes, in $3 \rightarrow 3$ kinematics,

$$
\frac{1}{2 \pi i} \frac{d}{d \ln \delta} \mathcal{W}_{3 \rightarrow 3}^{\mathrm{ns}}(1-\delta, v, v)=-\frac{1}{4} \Gamma_{1}(a) \exp \left[-\frac{\gamma_{K}(a)}{8}\left(\ln ^{2}|\delta|-L^{2}\right)\right] \mathcal{W}_{2}^{\mathrm{s}}(\delta),
$$




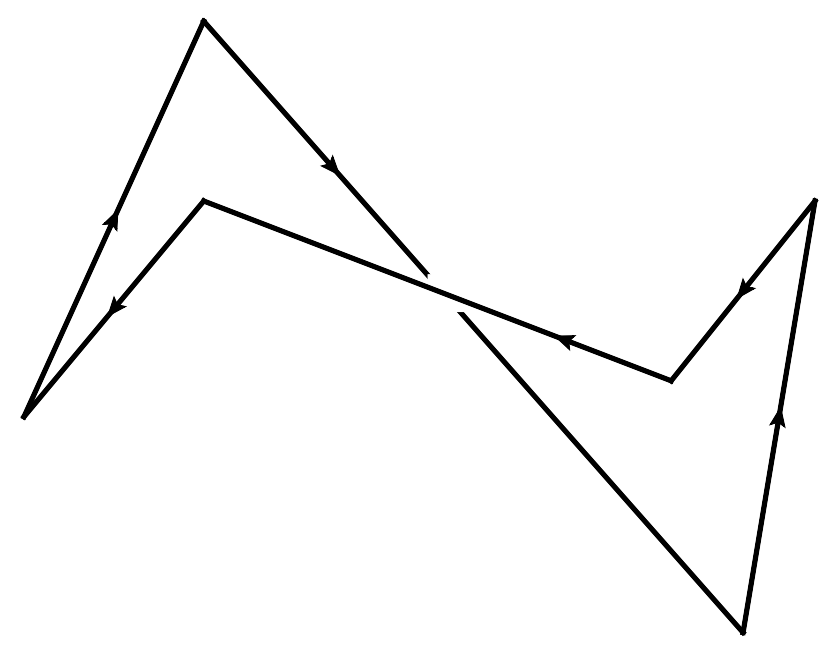

Figure 10. The two box Wilson loops that the hexagon mixes into in $3 \rightarrow 3$ kinematics both have Euclidean kinematics.

where $\mathcal{W}_{2}^{\mathrm{s}} \equiv W_{2} \times W_{\mathrm{f}}^{\mathrm{s}}$ is dual conformally invariant, but too singular to depend on $v$, so we write it as a function only of $\delta$. Thus all non-trivial kinematic dependence has been factored out in the exponential pre-factor of eq. (4.18).

We can also use eq. (2.37) to convert eq. (4.18) to one for $\mathcal{E}$ in $3 \rightarrow 3$ kinematics, since the relative factor between them is independent of $\delta$,

$$
\frac{1}{2 \pi i} \frac{d}{d \ln \delta} \mathcal{E}_{3 \rightarrow 3}(1-\delta, v, v)=-\frac{1}{4} \Gamma_{1}(a) \exp \left[-\frac{\gamma_{K}(a)}{8}\left(\ln ^{2}|\delta|-4 \zeta_{2}\right)\right] \mathcal{W}_{2}^{\mathrm{s}}(\delta) .
$$

This equation explains the $v$ independence of the singular terms in $\mathcal{E}_{3 \rightarrow 3}$ from 1 to 5 loops. It also explains why the singularities are purely in the imaginary part: the hexagon $3 \rightarrow 3$ kinematics we studied alternate between incoming and outgoing momenta, as one goes cyclically around the loop, as shown in figure 1(b) and also in figure 10. The two boxes that this hexagon factorizes into have Euclidean kinematics, in the sense that the invariants connecting adjacent legs (the so-called $s$ and $t$ channels for a scattering process) are both space-like. The only time-like invariant is in the $u$ channel involving diagonally opposite legs, but this invariant cannot produce cuts in the large $N_{c}$ limit, and so the box Wilson loops must be real. In other words, a factor of $\pi i$ comes from the cross anomalous dimension, and the remaining factors in eq. (4.19) are real.

Suppose we took eq. (4.15) for the $\delta$-dependence of $\mathcal{W}_{2}(\delta, v)$ seriously. Then we could combine it with eq. (4.17) and the observation that $\mathcal{W}_{2}^{\mathrm{s}}$ is independent of $v$ to fix $f(v)$ and conclude that

$$
\mathcal{W}_{2}^{\mathrm{s}}(\delta)=C(a) \exp \left[\frac{\bar{\Gamma}(a)}{2} \ln |\delta|\right]
$$

in $3 \rightarrow 3$ kinematics, where $C(a)$ is independent of both $v$ and $\delta$. Then we would find that eq. (4.18) becomes

$$
\frac{1}{2 \pi i} \frac{d}{d \ln \delta} \mathcal{W}_{3 \rightarrow 3}^{\mathrm{ns}}(1-\delta, v, v) \stackrel{?}{=}-\frac{1}{4} C(a) \Gamma_{1}(a) \exp \left[-\frac{\gamma_{K}(a)}{8}\left(\ln ^{2}|\delta|-L^{2}\right)+\frac{\bar{\Gamma}(a)}{2} \ln |\delta|\right] .
$$


In section 6 we will find that this expression is incompatible with explicit results, beginning at order $a^{4}$ and $\ln ^{3}|\delta|$. The leading-logarithmic terms in eq. (4.21) (terms of order $\left.a^{L} \ln ^{2 L-2}|\delta|\right)$ do have the correct form, but that is a tiny part of the available data.

It might be possible to compute the singularly-framed Wilson loop $\mathcal{W}_{2}^{\mathrm{s}}(\delta)$ directly, but we will not attempt to do so here. Instead, in the next section, we will use a matching between the self-crossing limit at small $v$ and a corner of the multi-Regge limit, in order to compute the left-hand side of eq. (4.18). We leave it to future work to evaluate $\mathcal{W}_{2}^{\mathrm{s}}(\delta)$ directly.

\section{Matching the self-crossing and multi-Regge limits}

We now understand the $v$-dependence of the singular terms in the MHV amplitude and associated Wilson loops in the self-crossing limit. We wish to use this information to evaluate the $\ln \delta$ terms to high loop orders for all values of $v$, using eq. (4.18) and an evaluation of $\mathcal{W}_{3 \rightarrow 3}^{\text {ns }}(v)$ at some value of $v$ to high orders. A convenient place to do this is for $v$ near zero, where it overlaps the multi-Regge limit.

The multi-Regge limit of six-gluon scattering in planar $\mathcal{N}=4 \mathrm{SYM}$ depends on a singular parameter $\delta$ and on a complex parameter, conventionally called $w$. (In order to minimize confusion between this $w$ and the cross ratio $w$ for generic kinematics, in this section we call the three cross ratios $\left(u_{1}, u_{2}, u_{3}\right)$.) The purpose of this section is first to identify the $w \rightarrow-1$ limit within multi-Regge kinematics with the $v \rightarrow 0$ limit within the self-crossing configuration. Then we evaluate the all-orders MRK formulae [49] in this limit, in order to provide an expression for the self-crossing configuration to high loop orders.

The MRK limit is defined in $2 \rightarrow 4$ kinematics by the analytic continuation $u_{1} \rightarrow$ $u_{1} e^{-2 \pi i}$ from the Euclidean region, followed by letting

$$
u_{1}=1-\delta, \quad u_{2}=\frac{\delta}{|1+w|^{2}}, \quad u_{3}=\frac{\delta|w|^{2}}{|1+w|^{2}},
$$

and taking $\delta \rightarrow 0$ with $\delta$ positive. In $3 \rightarrow 3$ kinematics, we first continue $u_{1} \rightarrow u_{1} e^{+2 \pi i}$, $u_{2} \rightarrow u_{2} e^{\pi i}, u_{3} \rightarrow u_{3} e^{\pi i}$ from the Euclidean region, and then take the same limit (5.1) but with $\delta$ negative, $\delta=-|\delta|$.

The self-crossing limit, in either $2 \rightarrow 4$ or $3 \rightarrow 3$ kinematics, is defined by the same analytic continuation as in the MRK case, followed by

$$
u_{1}=1-\delta, \quad u_{2}=v, \quad u_{3}=v .
$$

By comparing eqs. (5.1) and (5.2), we see that the overlap region is the limit $w \rightarrow-1$ of MRK, and the limit $v \rightarrow 0$ of self-crossing kinematics. The key relation for passing between the two limits is

$$
v=\frac{\delta}{|1+w|^{2}}, \quad \ln v=\ln \delta-\ln |1+w|^{2}
$$

for $2 \rightarrow 4$ kinematics, and

$$
|v|=\frac{|\delta|}{|1+w|^{2}}, \quad \ln |v|=\ln |\delta|-\ln |1+w|^{2}
$$

for $3 \rightarrow 3$ kinematics. 


\subsection{Self-crossing-MRK limit of the Wilson loop in $3 \rightarrow 3$ kinematics}

The behavior of the remainder function $R_{6}$ in the $2 \rightarrow 4$ MRK limit is $[42,46]$ :

$$
\begin{array}{r}
\left.\exp \left[R_{6}+i \pi \delta_{\mathrm{MRK}}\right]\right|_{\mathrm{MRK}, 2 \rightarrow 4}=\cos \pi \omega_{a b}+i \frac{a}{2} \sum_{n=-\infty}^{\infty}(-1)^{n}\left(\frac{w}{w^{*}}\right)^{\frac{n}{2}} \int_{-\infty}^{+\infty} \frac{d \nu}{\nu^{2}+\frac{n^{2}}{4}}|w|^{2 i \nu} \Phi_{\operatorname{Reg}}(\nu, n) \\
\times\left(-\frac{1}{1-u_{1}} \frac{|1+w|^{2}}{|w|}\right)^{\omega(\nu, n)}, \quad(5.5)
\end{array}
$$

where

$$
\begin{aligned}
\omega_{a b} & =\frac{1}{8} \gamma_{K}(a) \ln |w|^{2}, \\
\delta_{\mathrm{MRK}} & =\frac{1}{8} \gamma_{K}(a) \ln \frac{|w|^{2}}{|1+w|^{4}},
\end{aligned}
$$

and $\gamma_{K}(a)$ is the cusp anomalous dimension.

The behavior in the $3 \rightarrow 3$ kinematic region is obtained by letting $\ln \left(1-u_{1}\right) \rightarrow$ $\ln \left(u_{1}-1\right)-i \pi=\ln |\delta|-i \pi$ and performing a complex conjugation [39]:

$$
\begin{gathered}
\left.\exp \left[R_{6}-i \pi \delta_{\mathrm{MRK}}\right]\right|_{\mathrm{MRK}, 3 \rightarrow 3}=\cos \pi \omega_{a b}-i \frac{a}{2} \sum_{n=-\infty}^{\infty}(-1)^{n}\left(\frac{w}{w^{*}}\right)^{\frac{n}{2}} \int_{-\infty}^{+\infty} \frac{d \nu}{\nu^{2}+\frac{n^{2}}{4}}|w|^{2 i \nu} \Phi_{\operatorname{Reg}}(\nu, n) \\
\times\left(\frac{|1+w|^{2}}{|\delta||w|}\right)^{\omega(\nu, n)}
\end{gathered}
$$

In particular, there is no longer a phase in the last factor, so the real part in $3 \rightarrow 3$ kinematics is trivial,

$$
\left.\operatorname{Re} \exp \left[R_{6}-i \pi \delta_{\mathrm{MRK}}\right]\right|_{\mathrm{MRK}, 3 \rightarrow 3}=\cos \pi \omega_{a b}
$$

According to eq. (2.27), the nonsingularly-framed Wilson loop is related to $\exp \left[R_{6}\right]$ by multiplying by $\exp \left[\frac{\gamma_{K}}{8} X\right]$. We can use eq. (2.33) to evaluate $X$ in the self-crossing limit as $v \rightarrow 0$ (from the negative side):

$$
\left.X_{3 \rightarrow 3}^{\mathrm{ns}}\right|_{v \rightarrow 0}=2 \pi i \ln \left(\frac{|\delta|}{|v|}\right)=2 \pi i \ln |1+w|^{2} .
$$

Notice that as $w \rightarrow-1, \omega_{a b} \rightarrow 0$, while the phase $\delta_{\mathrm{MRK}}$ becomes $-\gamma_{K} / 4 \times \ln |1+w|^{2}$, so that

$$
\exp \left[-i \pi \delta_{\mathrm{MRK}}\right] \rightarrow \exp \left[\left.\frac{\gamma_{K}}{8} X_{3 \rightarrow 3}^{\mathrm{ns}}\right|_{v \rightarrow 0}\right] .
$$

Because these phases coincide, the $v \rightarrow 0$ limit of the $3 \rightarrow 3$ self-crossing configuration of the non-singularly-framed Wilson loop is purely imaginary - apart from the trivial term ' 1 ' arising from the limit of $\cos \pi \omega_{a b}$. It is given by the $\left(w, w^{*}\right) \rightarrow-1$ limit of eq. (5.8):

$$
\mathcal{W}_{3 \rightarrow 3}^{\mathrm{ns}}(v \rightarrow 0)=1-i \frac{a}{2} \sum_{n=-\infty}^{\infty}\left(\frac{w}{w^{*}}\right)^{\frac{n}{2}}(-1)^{n} \int_{-\infty}^{+\infty} \frac{d \nu}{\nu^{2}+\frac{n^{2}}{4}}|w|^{2 i \nu} \Phi_{\operatorname{Reg}}(\nu, n)|v|^{-\omega(\nu, n)} .
$$

Where $w$ and $w^{*}$ still appear in eq. (5.12), they are needed to regularize the sum and integral. 


\subsection{Evaluation of the Fourier-Mellin transform for $w \rightarrow-1$}

In this subsection we describe how to evaluate (most of) the terms in eq. (5.12) directly to high loop orders, in the limit $w \rightarrow-1$. While this paper was being written, ref. [51] appeared, which provides an efficient evaluation of the MRK limit to high loop orders for generic values of $\left(w, w^{*}\right)$ in terms of SVHPLs [77]. The two methods are complementary, in the sense that SVHPLs are not required for the limit $w \rightarrow-1$, so a fair amount of computational machinery can be bypassed. On the other hand, we will see that our method misses a few terms, for which we can use the results of ref. [51].

In ref. [49], the BFKL eigenvalue $\omega$ and impact factor $\Phi_{\text {Reg }}$ were computed to all orders using integrability. It is straightforward to obtain the perturbative expansions of $\omega$ and $\Phi_{\text {Reg }}$ to high orders, and insert them into eq. (5.12). Actually, it is better to trade the integral over $\nu$ for an integral over the rapidity $u$ which appears in ref. [49]. This saves one step because the functions $\omega$ and $\Phi_{\text {Reg }}$ are initially defined in terms of $u$ rather than $\nu$.

At any fixed loop order, $\omega$ and $\Phi_{\text {Reg }}$ (or alternatively the BFKL measure) are polynomials in the function $\psi(x)=d \ln \Gamma(x) / d x$ and its derivatives, where $x=1 \pm i u+n / 2$, together with rational functions of $u$ and $n$ and Riemann zeta values. To evaluate the integral over the real rapidity $u$, we deform it into the complex plane, where it has an infinite sequence of poles, at $i u=n / 2+m$ for non-negative $m$. We calculate the residues and then examine the behavior of the double sum in $n$ and $m$. We wish to pick up terms that contain at least one power each of $\ln (1+w)$ and $\ln \left(1+w^{*}\right)$. This divergent behavior as $\left(w, w^{*}\right) \rightarrow-1$ comes from the leading behavior of the summand as $m, n \rightarrow \infty$. We let $n=\hat{n}-m$, so that $\hat{n} \geq m$. The residues are all functions involving $\psi^{(p)}(m)$ or $\psi^{(p)}(1+\hat{n})$, combined with rational terms. However, the $\psi^{(p)}$ terms for $p>0$ always give power-suppressed terms as $m, n \rightarrow \infty$. Among the $\psi$ functions, we only need to keep $\psi(m) \approx \ln m$ and $\psi(1+\hat{n}) \approx \ln \hat{n}$.

After dropping all power-suppressed terms, we find that the double sum can be expressed solely in terms of

$$
S_{k}\left(w, w^{*}\right)=\sum_{\hat{n}=1}^{\infty} \frac{(-w)^{\hat{n}}}{\hat{n}} \sum_{m=1}^{\hat{n}-1} \frac{\left(-w^{*}\right)^{m}}{m}\left(\ln \hat{n}+\ln m+2 \gamma_{E}\right)^{k},
$$

for non-negative integers $k$, where $\gamma_{E}$ is the Euler-Mascheroni constant, plus a similar term with the roles of $\hat{n}$ and $m$ exchanged. When we add the term with $\hat{n} \leftrightarrow m$, we obtain an unrestricted sum over $\hat{n}$ and $m$, up to diagonal terms with $\hat{n}=m$, which can be dropped because they are not divergent. We then use the binomial theorem to decouple the two sums:

$$
S_{k}\left(w, w^{*}\right)=\sum_{p=0}^{k}\left(\begin{array}{l}
k \\
p
\end{array}\right) \hat{S}_{p}(w) \hat{S}_{k-p}\left(w^{*}\right)
$$

where

$$
\hat{S}_{p}(w)=\sum_{n=1}^{\infty} \frac{(-w)^{n}}{n}\left(\ln n+\gamma_{E}\right)^{p} .
$$

Next we extract the terms in eq. (5.15) containing positive powers of $\ln (1+w)$, for generic values of $p$,

$$
\hat{S}_{p}(w)=\sum_{\ell=1}^{p+1} d_{p, \ell} \ln ^{\ell}(1+w)+\mathcal{O}\left(\ln ^{0}(1+w)\right),
$$


where $d_{p, \ell}$ are some constants. We do this by taking the Mellin transform of both sides of eq. (5.16). Let $w=-z$, and consider

$$
\begin{aligned}
I_{p} & =\int_{0}^{1} d z z^{N-1} \hat{S}_{p}(-z)=\sum_{n=1}^{\infty} \frac{\left(\ln n+\gamma_{E}\right)^{p}}{n} \int_{0}^{1} d z z^{N+n-1} \\
& =\sum_{n=1}^{\infty} \frac{\left(\ln n+\gamma_{E}\right)^{p}}{n(n+N)}
\end{aligned}
$$

The large $N$ limit of the Mellin transform is sensitive only to the $z \rightarrow 1$ limit of the function being transformed. It is straightforward to approximate the sum over $n$ in eq. (5.17) by an integral, which can be evaluated in terms of classical polylogarithms. Then the desired limit as $N \rightarrow \infty$ can be taken, keeping only terms with positive powers of $\ln \hat{N} \equiv \ln N+\gamma_{E}$ multiplying $1 / N$. The first few values are

$$
\begin{aligned}
I_{0} & =\frac{1}{N} \ln \hat{N} \\
I_{1} & =\frac{1}{N}\left[\frac{1}{2} \ln ^{2} \hat{N}\right] \\
I_{2} & =\frac{1}{N}\left[\frac{1}{3} \ln ^{3} \hat{N}+2 \zeta_{2} \ln \hat{N}\right] \\
I_{3} & =\frac{1}{N}\left[\frac{1}{4} \ln ^{4} \hat{N}+3 \zeta_{2} \ln ^{2} \hat{N}\right] \\
I_{4} & =\frac{1}{N}\left[\frac{1}{5} \ln ^{5} \hat{N}+4 \zeta_{2} \ln ^{3} \hat{N}+42 \zeta_{4} \ln \hat{N}\right] .
\end{aligned}
$$

Notice that the $I_{p}$ obey

$$
\frac{d}{d N}\left[N I_{p}(N)\right]=p I_{p-1}(N)+\mathcal{O}\left(\frac{1}{N}\right) .
$$

This result can established by integration by parts in $n$. Hence the structure of the integrals $I_{p}(N)$ at large $N$ is dictated by this recursion relation, up to an integration constant, or equivalently the rational number $r_{m}$ multiplying $\zeta_{2 m} / N \times \ln \hat{N}$ in $I_{2 m}$. This sequence of numbers,

$$
r_{m}=2,42,1395,80010,7243425,957535425, \frac{348670597275}{2}, 41844302750250, \ldots,
$$

whose $m^{\text {th }}$ term always contains a factor of $2^{2 m-1}-1$, is given in turn by another recursion relation,

$$
\frac{r_{m}}{2^{2 m-1}-1}=\frac{m(2 m-1)}{2} \times \frac{r_{m-1}}{2^{2(m-1)-1}-1},
$$

which is valid for at least the first 35 terms.

We also need to perform the Mellin transform of $\ln ^{\ell}(1+w)=\ln ^{\ell}(1-z)$,

$$
M_{\ell} \equiv \int_{0}^{1} d z z^{N-1} \ln ^{\ell}(1-z)
$$


by using the formula,

$$
\int_{0}^{1} d z z^{N-1}(1-z)^{\alpha}=\frac{\Gamma(N) \Gamma(\alpha+1)}{\Gamma(N+\alpha+1)}
$$

differentiating $\ell$ times, then setting $\alpha=0$ and taking $N \rightarrow \infty$. The first few values are,

$$
\begin{aligned}
& M_{1}=-\frac{1}{N} \ln \hat{N} \\
& M_{2}=\frac{1}{N} \ln ^{2} \hat{N} \\
& M_{3}=\frac{1}{N}\left[-\ln ^{3} \hat{N}-3 \zeta_{2} \ln \hat{N}\right], \\
& M_{4}=\frac{1}{N}\left[\ln ^{4} \hat{N}+6 \zeta_{2} \ln ^{2} \hat{N}+8 \zeta_{3} \ln \hat{N}\right], \\
& M_{5}=\frac{1}{N}\left[-\ln ^{5} \hat{N}-10 \zeta_{2} \ln ^{3} \hat{N}-20 \zeta_{3} \ln ^{2} \hat{N}-\frac{135}{2} \zeta_{4} \ln \hat{N}\right],
\end{aligned}
$$

again omitting the $1 / N$ terms without logarithms. Notice that $M_{\ell}$ obeys a very similar recursion relation to $I_{i}$ :

$$
\frac{d}{d N}\left[N M_{\ell}(N)\right]=-\ell M_{\ell-1}(N)+\mathcal{O}\left(\frac{1}{N}\right)
$$

This result can established by integration by parts in $z$. It implies that

$$
\frac{d}{d N}(N M[f])=M\left[-d f / d L_{w}\right]+\mathcal{O}\left(\frac{1}{N}\right),
$$

for the Mellin transform $M[f]$ of any function $f$ that is a polynomial in $L_{w} \equiv \ln (1+w)$.

Using these results, we can rewrite $I_{p}$, the Mellin transform of $\hat{S}_{p}$, as a linear combination of $M_{\ell}$, the Mellin transforms of $\ln ^{\ell}(1+w)$, with $\zeta$ valued coefficients. Then $\hat{S}_{p}$ is given by the same linear combination of powers of $L_{w}$. The first few orders are given by:

$$
\begin{aligned}
& \hat{S}_{0}=-L_{w} \\
& \hat{S}_{1}=\frac{1}{2} L_{w}^{2} \\
& \hat{S}_{2}=-\frac{1}{3} L_{w}^{3}-\zeta_{2} L_{w}, \\
& \hat{S}_{3}=\frac{1}{4} L_{w}^{4}+\frac{3}{2} \zeta_{2} L_{w}^{2}+2 \zeta_{3} L_{w}, \\
& \hat{S}_{4}=-\frac{1}{5} L_{w}^{5}-2 \zeta_{2} L_{w}^{3}-4 \zeta_{3} L_{w}^{2}-\frac{27}{2} \zeta_{4} L_{w} .
\end{aligned}
$$

We observe that the $\hat{S}_{p}$ also obey a recursion relation,

$$
\frac{d}{d L_{w}} \hat{S}_{p}\left(L_{w}\right)=-p \hat{S}_{p-1}\left(L_{w}\right)+\mathcal{O}(1)
$$

which follows from eqs. (5.19) and (5.26). Hence the $\hat{S}_{p}$ are completely dictated by the coefficient of just the first power of $L_{w}$ at each order $p$. 
Inserting eq. (5.27) into eq. (5.13), we get similar formulas for $S_{k}\left(w, w^{*}\right)$, which we can rewrite as polynomials in $\ln |1+w|^{2}=L_{w}+L_{w^{*}} \equiv L_{|w|^{2}}$. For the first few orders, we find:

$$
\begin{aligned}
& S_{0}=\frac{1}{2}\left(L_{|w|^{2}}\right)^{2}, \\
& S_{1}=-\frac{1}{6}\left(L_{|w|^{2}}\right)^{3} \\
& S_{2}=\frac{1}{12}\left(L_{|w|^{2}}\right)^{4}+\zeta_{2}\left(L_{|w|^{2}}\right)^{2}, \\
& S_{3}=-\frac{1}{20}\left(L_{|w|^{2}}\right)^{5}-\zeta_{2}\left(L_{|w|^{2}}\right)^{3}-2 \zeta_{3}\left(L_{|w|^{2}}\right)^{2}, \\
& S_{4}=\frac{1}{30}\left(L_{|w|^{2}}\right)^{6}+\zeta_{2}\left(L_{|w|^{2}}\right)^{4}+\frac{8}{3} \zeta_{3}\left(L_{|w|^{2}}\right)^{3}+21 \zeta_{4}\left(L_{|w|^{2}}\right)^{2} .
\end{aligned}
$$

Again there is a recursion relation,

$$
\frac{d}{d L_{|w|^{2}}} S_{k}\left(L_{|w|^{2}}\right)=-k S_{k-1}\left(L_{|w|^{2}}\right)+\mathcal{O}\left(L_{|w|^{2}}\right)
$$

and the $S_{k}$ are determined by the coefficient of $\left(L_{|w|^{2}}\right)^{2}$ at each $k$.

After determining the $S_{k}$ to high orders, we insert them into the expression for the large $(\hat{n}, m)$ limit of the double sum for the MRK limit at each loop order. A divergence in both $w \rightarrow-1$ and $w^{*} \rightarrow-1$ is required in order to be able to neglect contributions to the double sum (5.13) from finite $\hat{n}$ and $m$. Therefore this method, although computationally very efficient, only determines the coefficients in front of $\ln ^{k}|1+w|^{2}$ for $k \geq 2$. It misses the coefficients in front of $\ln |1+w|^{2}$ and the constant term. On the other hand, using a complete basis of SVHPLs through weight 10, we were able to determine the complete MRK limits through five loops, in agreement with ref. [50]. We also obtained $g_{r}^{(L)}\left(w, w^{*}\right)$, the coefficient of $\ln ^{r} \delta$ in the MRK limit of the $L$-loop remainder function $R_{6}^{(L)}$, for $r \geq 2 L-11$ for $6 \leq L \leq 10$. Using this information, we could also determine the coefficients in front of $\ln |1+w|^{2}$ and the constant term for all terms with weight 10 or fewer.

After generating such results through nine loops, as a polynomial in $\ln |v|$ and $\ln |1+w|^{2}$, we used eq. (5.4) to rewrite it as a polynomial in $\ln |v|$ and $\ln |\delta|$. Then we imposed the further constraint that the result was consistent with the small $v$ limit of eq. (4.18), i.e. that the $v$-dependence was precisely proportional to $\exp \left[\frac{\gamma_{K}}{8} \ln ^{2}|v|\right]$. This constraint fixes additional terms. Finally we compared with the results of Broedel and Sprenger [51], which are complete through weight 13 . Their results were completely consistent with ours, and fixed all constants through weight 13 .

\section{$6 \quad$ Final result for singular terms}

In this section we present the final result for the singular terms in the expansion of the nonsingular framing of the hexagonal Wilson loop in the limit of $3 \rightarrow 3$ self-crossing kinematics, as $|\delta| \rightarrow 0$. The result can be written as,

$$
\frac{1}{2 \pi i} \frac{d \mathcal{W}_{3 \rightarrow 3}^{\mathrm{ns}}}{d \ln |\delta|}=\exp \left[-\frac{\gamma_{K}}{8}\left(\ln ^{2}|\delta|-L^{2}\right)\right] g\left(\ln |\delta|, \gamma_{K}\right),
$$


where $g$ depends only on $\ln |\delta|$ and the 't Hooft coupling. We choose to write the final result as an expansion in the cusp anomalous dimension $\gamma_{K}(a)$ instead of the coupling parameter $a$. We give the expansion of $\gamma_{K}(a)$ through 10 loops in appendix B.

The leading-ln $|\delta|$ terms in eq. (6.1) all come from the factor $\exp \left[-\gamma_{K} \ln ^{2}|\delta| / 8\right]$, multiplied by a factor of $\gamma_{K} / 8$, and keeping only the leading term in $\gamma_{K}(a)=4 a$ :

$$
\frac{a}{2} \int_{0}^{\ln |\delta|} d x \exp \left[-\frac{a}{2} x^{2}\right]=\frac{a}{2} \ln |\delta|-\frac{a^{2}}{12} \ln ^{3}|\delta|+\frac{a^{3}}{80} \ln ^{5}|\delta|-\frac{a^{4}}{672} \ln ^{7}|\delta|+\ldots
$$

Furthermore, we find that all of the purely even $\zeta$ terms in $g$, and many of the terms with single odd $\zeta$ values, can be captured by the following expression:

$$
\begin{aligned}
g_{0}\left(\ln |\delta|, \gamma_{K}\right) \equiv & \frac{\gamma_{K}}{8} \exp \left[\frac{\gamma_{K}}{2} \gamma_{E} \ln |\delta|\right] \frac{\Gamma\left(1+\frac{\gamma_{K}}{4} \ln |\delta|\right)}{\Gamma\left(1-\frac{\gamma_{K}}{4} \ln |\delta|\right)} \\
& \times\left\{1+\sum_{k=1}^{\infty} \frac{1}{k !}\left(-\frac{\gamma_{K}}{8}\right)^{k}\left[\psi^{(2 k-1)}\left(1+\frac{\gamma_{K}}{4} \ln |\delta|\right)-\psi^{(2 k-1)}\left(1-\frac{\gamma_{K}}{4} \ln |\delta|\right)\right]\right\}
\end{aligned}
$$

where $\psi^{(k)}(x)$ denotes the $k^{\text {th }}$ derivative of $\psi(x)=d \ln \Gamma(x) / d x$, and $\gamma_{E}$ is the EulerMascheroni constant.

Writing the full function $g$ as $g_{0}$ plus a residual term $g_{1}$,

$$
g\left(\ln |\delta|, \gamma_{K}\right)=g_{0}\left(\ln |\delta|, \gamma_{K}\right)+g_{1}\left(\ln |\delta|, \gamma_{K}\right),
$$

we give the expansion of $g_{1}$, which starts at three loops:

$$
g_{1}\left(\ln |\delta|, \gamma_{K}\right)=\frac{1}{4} \sum_{L=3}^{\infty} c^{(L)}\left(\frac{\gamma_{K}}{4}\right)^{L}
$$

where

$$
\begin{aligned}
c^{(3)}= & -6 \zeta_{3} \ln |\delta|, \\
c^{(4)}= & \left(20 \zeta_{5}-8 \zeta_{2} \zeta_{3}\right) \ln |\delta|-2\left(\zeta_{3}\right)^{2}, \\
c^{(5)}= & 5\left(\zeta_{3}\right)^{2} \ln ^{2}|\delta|-\left(\frac{175}{2} \zeta_{7}-50 \zeta_{2} \zeta_{5}\right) \ln |\delta|+25 \zeta_{3} \zeta_{5}+\zeta_{2}\left(\zeta_{3}\right)^{2}, \\
c^{(6)}= & -\left(96 \zeta_{3} \zeta_{5}+8 \zeta_{2}\left(\zeta_{3}\right)^{2}\right) \ln ^{2}|\delta|+\left(441 \zeta_{9}-315 \zeta_{2} \zeta_{7}+72 \zeta_{4} \zeta_{5}-24\left(\zeta_{3}\right)^{3}\right) \ln |\delta| \\
& -194 \zeta_{3} \zeta_{7}-99\left(\zeta_{5}\right)^{2}-12 \zeta_{4}\left(\zeta_{3}\right)^{2}-16 \zeta_{2} \zeta_{3} \zeta_{5}, \\
c^{(7)}= & \left(\frac{164}{3} \zeta_{3} \zeta_{5}+\frac{16}{3} \zeta_{2}\left(\zeta_{3}\right)^{2}\right) \ln ^{4}|\delta|+\frac{103}{3}\left(\zeta_{3}\right)^{3} \ln ^{3}|\delta| \\
& +\left(\frac{1985}{2} \zeta_{3} \zeta_{7}+496\left(\zeta_{5}\right)^{2}+40 \zeta_{4}\left(\zeta_{3}\right)^{2}+114 \zeta_{2} \zeta_{3} \zeta_{5}\right) \ln { }^{2}|\delta| \\
& +\left(-\frac{4851}{2} \zeta_{11}+2058 \zeta_{2} \zeta_{9}-882 \zeta_{4} \zeta_{7}+697\left(\zeta_{3}\right)^{2} \zeta_{5}+11 \zeta_{2}\left(\zeta_{3}\right)^{3}\right) \ln |\delta| \\
& +135 \zeta_{2} \zeta_{3} \zeta_{7}+108 \zeta_{4} \zeta_{3} \zeta_{5}+48 \zeta_{2}\left(\zeta_{5}\right)^{2}+1818 \zeta_{5} \zeta_{7}+1869 \zeta_{3} \zeta_{9}+27\left(\zeta_{3}\right)^{4}, \\
c^{(8)}= & -\left[\frac{28}{5} \zeta_{3} \zeta_{5} \ln ^{6}|\delta|+\frac{46}{3}\left(\zeta_{3}\right)^{3} \ln ^{5}|\delta|+\left(\frac{2225}{3} \zeta_{3} \zeta_{7}+384\left(\zeta_{5}\right)^{2}+\frac{244}{3} \zeta_{2} \zeta_{3} \zeta_{5}\right) \ln ^{4}|\delta|\right.
\end{aligned}
$$




$$
\begin{aligned}
& +\left(\frac{3806}{3}\left(\zeta_{3}\right)^{2} \zeta_{5}+\frac{206}{3} \zeta_{2}\left(\zeta_{3}\right)^{3}\right) \ln ^{3}|\delta| \\
& +\left(239\left(\zeta_{3}\right)^{4}+925 \zeta_{2} \zeta_{3} \zeta_{7}+400 \zeta_{2}\left(\zeta_{5}\right)^{2}+428 \zeta_{3} \zeta_{4} \zeta_{5}+11739 \zeta_{3} \zeta_{9}+11156 \zeta_{5} \zeta_{7}\right) \ln ^{2}|\delta| \\
& +\left(-14157 \zeta_{13}+13860 \zeta_{2} \zeta_{11}-8484 \zeta_{4} \zeta_{9}+540 \zeta_{6} \zeta_{7}+474 \zeta_{2}\left(\zeta_{3}\right)^{2} \zeta_{5}+\frac{15537}{2}\left(\zeta_{3}\right)^{2} \zeta_{7}\right. \\
& \left.\left.+7625 \zeta_{3}\left(\zeta_{5}\right)^{2}+238 \zeta_{4}\left(\zeta_{3}\right)^{3}\right) \ln |\delta|+c_{0}^{(8)}\right]
\end{aligned}
$$

and

$$
\begin{aligned}
c^{(9)}= & \frac{4}{3}\left(\zeta_{3}\right)^{3} \ln ^{7}|\delta|+\left(88\left(\zeta_{5}\right)^{2}+\frac{16}{5} \zeta_{2} \zeta_{3} \zeta_{5}+152 \zeta_{3} \zeta_{7}\right) \ln ^{6}|\delta|+\left(\frac{3244}{5}\left(\zeta_{3}\right)^{2} \zeta_{5}+\frac{64}{3} \zeta_{2}\left(\zeta_{3}\right)^{3}\right) \ln ^{5}|\delta| \\
& +\left(\frac{1043}{4}\left(\zeta_{3}\right)^{4}+690 \zeta_{2} \zeta_{3} \zeta_{7}-48 \zeta_{4} \zeta_{3} \zeta_{5}+10626 \zeta_{3} \zeta_{9}+10155 \zeta_{5} \zeta_{7}+300 \zeta_{2}\left(\zeta_{5}\right)^{2}\right) \ln ^{4}|\delta| \\
& +\left(\frac{5639}{3} \zeta_{2}\left(\zeta_{3}\right)^{2} \zeta_{5}+\frac{203707}{12}\left(\zeta_{3}\right)^{2} \zeta_{7}+16598 \zeta_{3}\left(\zeta_{5}\right)^{2}+128 \zeta_{4}\left(\zeta_{3}\right)^{3}\right) \ln ^{3}|\delta| \\
& +c_{2}^{(9)} \ln ^{2}|\delta| \\
& +\left(-\frac{2760615}{32} \zeta_{15}+\frac{382239}{4} \zeta_{2} \zeta_{13}-\frac{601425}{8} \zeta_{4} \zeta_{11}+\frac{20547}{2} \zeta_{6} \zeta_{9}+84 \zeta_{6}\left(\zeta_{3}\right)^{3}\right. \\
& \quad+\frac{10301}{2} \zeta_{4}\left(\zeta_{3}\right)^{2} \zeta_{5}+\frac{24569}{4} \zeta_{2}\left(\zeta_{3}\right)^{2} \zeta_{7}+5585 \zeta_{2} \zeta_{3}\left(\zeta_{5}\right)^{2}+30885\left(\zeta_{5}\right)^{3} \\
& \left.\quad+101668\left(\zeta_{3}\right)^{2} \zeta_{9}+\frac{757873}{4} \zeta_{3} \zeta_{5} \zeta_{7}+917\left(\zeta_{3}\right)^{5}\right) \ln |\delta| \\
& +c_{0}^{(9)},
\end{aligned}
$$

where $c_{0}^{(8)}, c_{2}^{(9)}$ and $c_{0}^{(9)}$ are linear combinations of multiple zeta values of weight 14,14 and 16 , respectively. We have some partial information about their rational-number coefficients; in particular,

$$
\begin{aligned}
2 c_{2}^{(9)}-c_{0}^{(8)}= & 339327 \zeta_{3} \zeta_{11}+307248 \zeta_{5} \zeta_{9}+\frac{1174875}{8}\left(\zeta_{7}\right)^{2}+18648 \zeta_{2} \zeta_{3} \zeta_{9}+13431 \zeta_{2} \zeta_{5} \zeta_{7} \\
& +1102 \zeta_{2}\left(\zeta_{3}\right)^{4}+5058 \zeta_{4} \zeta_{3} \zeta_{7}+2271 \zeta_{4}\left(\zeta_{5}\right)^{2}-1080 \zeta_{6} \zeta_{3} \zeta_{5}+26914\left(\zeta_{3}\right)^{3} \zeta_{5} .
\end{aligned}
$$

No terms in $g_{1}$ have only even $\zeta$ values in them (i.e. pure powers of $\pi$ ). Furthermore, the only terms in $g_{1}$ that have a single odd $\zeta$ value are those with a single power of $\ln |\delta|$. All the terms with a single odd $\zeta$ value and multiple powers of $\ln |\delta|$ have been absorbed into $g_{0}$.

We also attempted to fit the explicit results for the function $g$ to the consequences of eq. (4.21):

$$
g\left(\ln |\delta|, \gamma_{K}\right) \stackrel{?}{=} \hat{C}\left(\gamma_{K}\right) \exp \left[\frac{\bar{\Gamma}\left(\gamma_{K}\right)}{2} \ln |\delta|\right]
$$

where $\hat{C}\left(\gamma_{K}\right)=-C(a) \Gamma_{1}(a) / 4$ and $\bar{\Gamma}\left(\gamma_{K}\right)$ are taken to be arbitrary functions of the cusp anomalous dimension (i.e. of the coupling $a$ ). Expanding around $\gamma_{K}=0$, we find that $\hat{C}\left(\gamma_{K}\right) \propto \gamma_{K}+\mathcal{O}\left(\gamma_{K}^{2}\right), \bar{\Gamma}\left(\gamma_{K}\right) \propto \gamma_{K}^{2}+\mathcal{O}\left(\gamma_{K}^{3}\right)$, and then it becomes impossible to fit the true $g\left(\ln |\delta|, \gamma_{K}\right)$ with eq. (6.14), beginning with the $\gamma_{K}^{4}$ term. At this order, $g$ contains $\ln ^{3}|\delta|$ with a nonzero coefficient proportional to $\zeta_{3}$, while the $\ln ^{3}|\delta|$ coefficient vanishes in the ansatz (6.14). 
Despite this difficulty, the relative simplicity of the terms in $g_{1}$ with more than one power of $\ln |\delta|$ suggests that these terms might be on a different footing from the linear terms in $\ln |\delta|$, and might have a relatively simple kinematical origin. It will be interesting to investigate the structure of $g\left(\ln |\delta|, \gamma_{K}\right)$ further in the future, and to see what role the cross anomalous dimension in planar $\mathcal{N}=4 \mathrm{SYM}$ might play.

\section{Conclusions and outlook}

In this paper we observed that the duality between scattering amplitudes and Wilson loops in planar $\mathcal{N}=4 \mathrm{SYM}$ maps a configuration that mimics double-parton-scattering into a self-crossing limit of the relevant Wilson loop. We observed that helicity selection rules, especially $J_{z}$ conservation, are related to the finiteness of the NMHV six-gluon amplitude in $2 \rightarrow 4$ kinematics in QCD, $\mathcal{N}=1$ or $\mathcal{N}=4 \mathrm{SYM}$ at one loop. Beyond one loop, there are logarithmic divergences, although the transcendental function $E$ is nonsingular at the leading power of $\delta$. The transcendental function entering the MHV amplitude, in contrast, can be and is singular. We presented explicit results through five loops, including the nonsingular terms. Then we studied the structure of the singular terms, and determined their kinematic dependence exactly, using an evolution equation for self-crossing Wilson loops in the large $N_{c}$ limit. In particular, we explained the surprising $v$-independence of the singular terms in $\mathcal{E}_{3 \rightarrow 3}$ in eq. (4.19). Finally, we exploited the overlap between the self-crossing limit and the multi-Regge limit as $v \rightarrow 0$, to determine the answer in that limit to nine loops, up to a couple of zeta-valued constants. The leading-logarithmic part of this formula, eq. (6.2), gives a simple representation for the leading logs studied earlier [28-30] to all loop orders.

We can use similar methods to study self-crossing limits of the $n$-point amplitude for $n>6$. For example, in appendix E we identify the self-crossing configuration for the seven-point case, in which the Wilson loop factorizes into the product of a box and a pentagon. This configuration is a four-parameter subspace of the general six-parameter space of dual conformal invariants. (Naively, there are seven dual conformal invariants in the seven-point case, related by the seven-fold cyclic symmetry; however in four dimensional spacetime they obey a single Gram determinantal constraint.) The same arguments given above suggest that the dependence on the four surviving nonsingular parameters in this case can be determined exactly in terms of the cusp anomalous dimension and a suitable one-loop function. The dependence on the singular parameter should be essentially the same as in the six-point case. This information will be useful in constructing or checking the full seven-point MHV amplitude at higher loop orders; it is currently known at the function level through two loops [78], and at symbol level through three loops [79].

Beginning with the eight-point amplitude, multiple types of single self-crossings are possible, corresponding to different partitions of the final momenta into two sets of momenta, and there can also be more than one self-crossing. For the single self-crossing, one always loses two degrees of freedom, because two separate transverse coordinates are constrained. For $n=6$, the three cross ratios $(u, v, w)$ reduce to one, $v$. For $n=7$, the six independent $u_{i}$ reduce to the four $u_{1}, u_{2}, u_{5} u_{6}$ given in appendix E. For larger $n$ there will be $(3 n-15)-2=3 n-17$ cross ratios left for a single self-crossing, and correspondingly 
fewer for multiple self-crossings. Even when the subprocesses have their own dual conformal cross ratios, there will still be additional parameters, and the functional dependence on those parameters should be calculable using factorization arguments of the kind used here.

Similarly, studies of non-MHV amplitudes for $n>6$ in these limits should be possible, using the correspondence with super-Wilson-loops [80, 81]. The finiteness of the one-loop NMHV six-gluon amplitude is clearly special to $n=6$ : it is easy to add an additional final-state gluon with negative helicity to the configurations in figure 3 to convert them to valid NMHV seven-point configurations. It would be quite interesting to combine some of the ideas and results of this paper with the recent general analysis of Landau singularities for $\mathcal{N}=4$ SYM amplitudes in ref. [9].

In summary, the self-crossing limit and its relation to kinematical configurations that mimic double-parton scattering should provide a rich playground for further investigations of the analytic behavior of multi-loop amplitudes, in both planar $\mathcal{N}=4 \mathrm{SYM}$ and more general theories such as QCD.

\section{Acknowledgments}

We are grateful to Benjamin Basso, Andrei Belitsky, Johannes Broedel, Simon CaronHuot, John Joseph Carrasco, Claude Duhr, Matt von Hippel, Gregory Korchemsky, Andrew McLeod, Tom Melia, Amit Sever, Georgios Papathanasiou, Mads Søgaard, Martin Sprenger, Pedro Vieira and Jara Trnka for useful discussions and comments. We would particularly like to thank Johannes Broedel and Martin Sprenger for providing us with the $w \rightarrow-1$ limit of their high-loop order MRK expressions, as well as Benjamin Basso, Simon Caron-Huot, Amit Sever and Pedro Vieira for pointing out an error in the NMHV discussion in the first version of this paper. This research was supported by the US Department of Energy under contract DE-AC02-76SF00515 and grant DE-SC0011632, by the Walter Burke Institute, by the Gordon and Betty Moore Foundation through Grant No. 776 to the Caltech Moore Center for Theoretical Cosmology and Physics. LD thanks Caltech, the Aspen Center for Physics and the NSF Grant \#1066293 for hospitality. Figures in this paper were made with JAXODRAW [82, 83], based on AxODRAW [84].

\section{A Self-crossing kinematics}

In this appendix we describe the kinematics of $2 \rightarrow 4$ scattering in the self-crossing or double-parton-scattering-like limit. Then we do the same for the analogous $3 \rightarrow 3$ scattering configuration.

\section{A.1 $\quad 2 \rightarrow 4$ kinematics}

For the $2 \rightarrow 4$ scattering configuration shown in figure 1 (a) we consider

$$
\begin{aligned}
k_{3}+k_{6} & \rightarrow k_{1}+k_{2}+k_{4}+k_{5}, \\
(1-x) k_{3}+(1-y) k_{6} & \rightarrow k_{1}+k_{2} \\
x k_{3}+y k_{6} & \rightarrow k_{4}+k_{5} .
\end{aligned}
$$


Incoming gluons 3 and 6 split into collinear pairs with momentum fractions $x$ and $1-x$, and $y$ and $1-y$, respectively. These pairs then undergo $2 \rightarrow 2$ scatterings into final state gluons $1,2,4$ and 5 .

We work in the center-of-mass (CM) frame, and take the spatial components of $k_{3}$ to be in the positive $z$ direction, while those of $k_{6}$ are in the negative $z$ direction. Momenta $k_{1}$ and $k_{2}$ are in the $x z$-plane, while $k_{4}$ and $k_{5}$ are rotated out of this plane by an azimuthal angle $\phi$. Incoming momenta are labeled by the negative of the true momentum, so that $\sum_{i=1}^{6} k_{i}=0$. Writing $k_{i}^{\mu}=\left(k_{i}^{t}, k_{i}^{x}, k_{i}^{y}, k_{i}^{z}\right)$, we have:

$$
\begin{aligned}
& k_{1}=\left(E_{1}, E_{1} \sin \theta_{1}, 0,-E_{1} \cos \theta_{1}\right), \\
& k_{2}=\left(E_{2},-E_{2} \sin \theta_{2}, 0,-E_{2} \cos \theta_{2}\right), \\
& k_{3}=\left(-\frac{1}{2} \sqrt{s_{36}}, 0,0,-\frac{1}{2} \sqrt{s_{36}}\right), \\
& k_{4}=\left(E_{4}, E_{4} \sin \theta_{4} \cos \phi, E_{4} \sin \theta_{4} \sin \phi,-E_{4} \cos \theta_{4}\right), \\
& k_{5}=\left(E_{5},-E_{5} \sin \theta_{5} \cos \phi,-E_{5} \sin \theta_{5} \sin \phi,-E_{5} \cos \theta_{5}\right), \\
& k_{6}=\left(-\frac{1}{2} \sqrt{s_{36}}, 0,0, \frac{1}{2} \sqrt{s_{36}}\right) .
\end{aligned}
$$

Momentum conservation for the $2 \rightarrow 2$ subprocesses in eqs. (A.31) and (A.32) implies that

$$
\begin{aligned}
s_{12} & =(1-x)(1-y) s_{36}, \\
s_{45} & =x y s_{36} \\
s_{123} & =-x(1-y) s_{36} \\
s_{345} & =-y(1-x) s_{36} \\
x s_{34} & =y s_{56} \\
(1-x) s_{23} & =(1-y) s_{61} .
\end{aligned}
$$

Inserting eq. (A.4) into these relations, we can express the energies $E_{i}$ and two of the angles in eq. (A.4) in terms of the momentum fractions $x$ and $y$, and the other two polar angles:

$$
\begin{aligned}
E_{1} & =\sqrt{s_{36}} \frac{(1-x)(1-y)}{\left(1+\cos \theta_{1}\right)(1-x)+\left(1-\cos \theta_{1}\right)(1-y)}, \\
E_{2} & =\frac{\sqrt{s_{36}}}{2} \frac{\left(1+\cos \theta_{1}\right)(1-x)^{2}+\left(1-\cos \theta_{1}\right)(1-y)^{2}}{\left(1+\cos \theta_{1}\right)(1-x)+\left(1-\cos \theta_{1}\right)(1-y)}, \\
\cos \theta_{2} & =\frac{\left(1-\cos \theta_{1}\right)(1-y)^{2}-\left(1+\cos \theta_{1}\right)(1-x)^{2}}{\left(1+\cos \theta_{1}\right)(1-x)^{2}+\left(1-\cos \theta_{1}\right)(1-y)^{2}}, \\
\sin \theta_{2} & =\frac{2(1-x)(1-y) \sin \theta_{1}}{\left(1+\cos \theta_{1}\right)(1-x)^{2}+\left(1-\cos \theta_{1}\right)(1-y)^{2}}, \\
E_{4} & =\frac{\sqrt{s_{36}}}{2} \frac{\left(1+\cos \theta_{5}\right) x^{2}+\left(1-\cos \theta_{5}\right) y^{2}}{\left(1+\cos \theta_{5}\right) x+\left(1-\cos \theta_{5}\right) y}, \\
E_{5} & =\sqrt{s_{36}} \frac{x y}{\left(1+\cos \theta_{5}\right) x+\left(1-\cos \theta_{5}\right) y},
\end{aligned}
$$




$$
\begin{aligned}
\cos \theta_{4} & =\frac{\left(1-\cos \theta_{5}\right) y^{2}-\left(1+\cos \theta_{5}\right) x^{2}}{\left(1+\cos \theta_{5}\right) x^{2}+\left(1-\cos \theta_{5}\right) y^{2}}, \\
\sin \theta_{4} & =\frac{2 x y \sin \theta_{5}}{\left(1+\cos \theta_{5}\right) x^{2}+\left(1-\cos \theta_{5}\right) y^{2}} .
\end{aligned}
$$

It's convenient to trade the angles $\theta_{1}$ and $\theta_{5}$ in the overall $\mathrm{CM}$ frame for the polar angles in the $\mathrm{CM}$ frames for the respective $2 \rightarrow 2$ subprocesses (A.2) and (A.3), which we call $\theta_{A}$ and $\theta_{B}$, respectively:

$$
\begin{aligned}
-\frac{(1-x) s_{23}}{s_{12}} & =\frac{1-\cos \theta_{A}}{2}=\frac{(1-y)\left(1-\cos \theta_{1}\right)}{\left(1+\cos \theta_{1}\right)(1-x)+\left(1-\cos \theta_{1}\right)(1-y)}, \\
-\frac{x s_{34}}{s_{45}} & =\frac{1-\cos \theta_{B}}{2}=\frac{y\left(1-\cos \theta_{5}\right)}{\left(1+\cos \theta_{5}\right) x+\left(1-\cos \theta_{5}\right) y} .
\end{aligned}
$$

Solving for $\theta_{1}$ and $\theta_{5}$, we have,

$$
\begin{aligned}
\cos \theta_{1} & =1-\frac{2(1-x)\left(1-c_{A}\right)}{\left(1-c_{A}\right)(1-x)+\left(1+c_{A}\right)(1-y)}, \\
\sin \theta_{1} & =\frac{2 s_{A} \sqrt{(1-x)(1-y)}}{\left(1-c_{A}\right)(1-x)+\left(1+c_{A}\right)(1-y)}, \\
\cos \theta_{5} & =1-\frac{2 x\left(1-c_{B}\right)}{\left(1-c_{B}\right) x+\left(1+c_{B}\right) y}, \\
\sin \theta_{5} & =\frac{2 s_{B} \sqrt{x y}}{\left(1-c_{B}\right) x+\left(1+c_{B}\right) y},
\end{aligned}
$$

where $c_{A, B}=\cos \theta_{A, B}$ and $s_{A, B}=\sin \theta_{A, B}$.

Of the four invariants appearing in the cross ratio $v=s_{23} s_{56} /\left(s_{234} s_{123}\right)$, three are simply related to the invariants for the individual $2 \rightarrow 2$ subprocesses. For example, $(1-x) s_{23}=(1-y) s_{16}$ is the $t$-channel invariant for subprocess $A$, so it is simple to relate it to the $u$-channel invariant $(1-x) s_{13}$, the $s$-channel invariant $s_{12}$ and the CM scattering angle $\theta_{A}$. The only invariant that straddles two subprocesses is $s_{234}=s_{23}+s_{34}+s_{24}$, and it does so only through $s_{24}$. Thus the dependence of $v$ on the azimuthal angle enters only through $s_{24}$.

It is convenient to normalize all invariants by $s_{36}$. Computing $s_{24} / s_{36}$ from eq. (A.4) and using eqs. (A.18) and (A.24) to express the result in terms of $x, y, \theta_{A}, \theta_{B}$ and $\phi$, we find that

$$
\frac{s_{24}}{s_{36}}=\frac{1}{4}\left[x(1-y)\left(1-c_{A}\right)\left(1+c_{B}\right)+y(1-x)\left(1+c_{A}\right)\left(1-c_{B}\right)+2 \sqrt{x y(1-x)(1-y)} s_{A} s_{B} \cos \phi\right] .
$$

The cross ratio $v$ can be written in terms of $s_{24} / s_{36}$ as,

$$
v=-\frac{\left(1-c_{A}\right)\left(1-c_{B}\right)}{4\left[s_{24} / s_{36}-\frac{1}{2}\left(1-c_{A}\right)(1-y)-\frac{1}{2}\left(1-c_{B}\right) y\right]} .
$$

After inserting eq. (A.25), we find that

$$
\frac{1}{v}-1=\frac{\left(1+c_{A}\right)\left(1-c_{B}\right) x y+\left(1-c_{A}\right)\left(1+c_{B}\right)(1-x)(1-y)-2 \sqrt{x y(1-x)(1-y)} s_{A} s_{B} \cos \phi}{\left(1-c_{A}\right)\left(1-c_{B}\right)}
$$




$$
\begin{aligned}
=\frac{1}{\left(1-c_{A}\right)\left(1-c_{B}\right)}\{ & {\left[\sqrt{\left(1+c_{A}\right)\left(1-c_{B}\right) x y}-\sqrt{\left(1-c_{A}\right)\left(1+c_{B}\right)(1-x)(1-y)}\right]^{2} } \\
& \left.+2 \sqrt{x y(1-x)(1-y)} s_{A} s_{B}(1-\cos \phi)\right\} .
\end{aligned}
$$

The second form (A.27) makes manifest that for $2 \rightarrow 4$ scattering,

$$
\frac{1}{v}-1 \geq 0, \quad \text { or } \quad 0 \leq v \leq 1
$$

The $v=0$ limit is only achieved when the denominator of eq. (A.27) vanishes. This happens when one of the two $2 \rightarrow 2$ subprocesses becomes collinear, either $\theta_{A}$ or $\theta_{B} \rightarrow 0$. The $v=1$ limit requires the numerator of eq. (A.27) to vanish, which implies that the event is planar,

$$
v=1 \quad \Leftrightarrow \quad \phi=0, \quad y=\frac{\left(1-c_{A}\right)\left(1+c_{B}\right)(1-x)}{\left(1+c_{A}\right)\left(1-c_{B}\right) x+\left(1-c_{A}\right)\left(1+c_{B}\right)(1-x)} .
$$

\section{A.2 $3 \rightarrow 3$ kinematics}

For the $3 \rightarrow 3$ scattering configuration shown in figure $1(\mathrm{~b})$ we consider

$$
\begin{aligned}
k_{1}+k_{3}+k_{5} & \rightarrow k_{2}+k_{4}+k_{6}, \\
k_{1}+(1-x) k_{3} & \rightarrow k_{2}+(1-y) k_{6}, \\
k_{5}+x k_{3} & \rightarrow k_{4}+y k_{6} .
\end{aligned}
$$

Gluon 3 splits into two partons, one of which collides with gluon 1 and the other with gluon 5. The products of those two collisions are gluons 2 and 4, and two more gluons. The latter two gluons then merge into gluon 6 .

Because $k_{3}$ is incoming and $k_{6}$ is outgoing, $s_{36}$ is negative. We can choose a "brick-wall frame" for these two momenta, in which $k_{3}$ is in the positive $z$ direction, while $k_{6}$ is in the negative $z$ direction, with $k_{6}^{z}=-k_{3}^{z}$. As in the $2 \rightarrow 4$ case, we take momenta $k_{1}$ and $k_{2}$ to lie in the $x z$-plane, while $k_{4}$ and $k_{5}$ are rotated out of this plane by an azimuthal angle $\phi$. We parametrize the momenta as:

$$
\begin{aligned}
& k_{1}=\left(-E_{1}, E_{1} \sin \theta_{1}, 0, E_{1} \cos \theta_{1}\right), \\
& k_{2}=\left(E_{2},-E_{2} \sin \theta_{2}, 0,-E_{2} \cos \theta_{2}\right), \\
& k_{3}=\left(-\frac{1}{2} \sqrt{-s_{36}}, 0,0,-\frac{1}{2} \sqrt{-s_{36}}\right), \\
& k_{4}=\left(E_{4}, E_{4} \sin \theta_{4} \cos \phi, E_{4} \sin \theta_{4} \sin \phi,-E_{4} \cos \theta_{4}\right), \\
& k_{5}=\left(-E_{5},-E_{5} \sin \theta_{5} \cos \phi,-E_{5} \sin \theta_{5} \sin \phi, E_{5} \cos \theta_{5}\right), \\
& k_{6}=\left(\frac{1}{2} \sqrt{-s_{36}}, 0,0,-\frac{1}{2} \sqrt{-s_{36}}\right) .
\end{aligned}
$$

Momentum conservation for the $2 \rightarrow 2$ subprocesses in eqs. (A.31) and (A.32) implies that kinematic relations in eqs. (A.5)-(A.10) still hold. Inserting eq. (A.33) into these 
relations, we again express the energies $E_{i}$ and two of the angles in eq. (A.33) in terms of the momentum fractions $x$ and $y$, and the other two polar angles:

$$
\begin{aligned}
E_{1} & =\sqrt{-s_{36}} \frac{(1-x)(1-y)}{\left(1+\cos \theta_{1}\right)(1-x)-\left(1-\cos \theta_{1}\right)(1-y)}, \\
E_{2} & =\frac{\sqrt{-s_{36}}}{2} \frac{\left(1+\cos \theta_{1}\right)(1-x)^{2}+\left(1-\cos \theta_{1}\right)(1-y)^{2}}{\left(1+\cos \theta_{1}\right)(1-x)-\left(1-\cos \theta_{1}\right)(1-y)}, \\
\cos \theta_{2} & =\frac{\left(1-\cos \theta_{1}\right)(1-y)^{2}-\left(1+\cos \theta_{1}\right)(1-x)^{2}}{\left(1+\cos \theta_{1}\right)(1-x)^{2}+\left(1-\cos \theta_{1}\right)(1-y)^{2}}, \\
\sin \theta_{2} & =\frac{2(1-x)(1-y) \sin \theta_{1}}{\left(1+\cos \theta_{1}\right)(1-x)^{2}+\left(1-\cos \theta_{1}\right)(1-y)^{2}}, \\
E_{4} & =\frac{\sqrt{-s_{36}}}{2} \frac{\left(1+\cos \theta_{5}\right) x^{2}+\left(1-\cos \theta_{5}\right) y^{2}}{\left(1+\cos \theta_{5}\right) x-\left(1-\cos \theta_{5}\right) y}, \\
E_{5} & =\sqrt{-s_{36}} \frac{x y}{\left(1+\cos \theta_{5}\right) x-\left(1-\cos \theta_{5}\right) y}, \\
\cos \theta_{4} & =\frac{\left(1-\cos \theta_{5}\right) y^{2}-\left(1+\cos \theta_{5}\right) x^{2}}{\left(1+\cos \theta_{5}\right) x^{2}+\left(1-\cos \theta_{5}\right) y^{2}}, \\
\sin \theta_{4} & =\frac{2 x y \sin \theta_{5}}{\left(1+\cos \theta_{5}\right) x^{2}+\left(1-\cos \theta_{5}\right) y^{2}} .
\end{aligned}
$$

Again we trade the angles $\theta_{1}$ and $\theta_{5}$ in the brick-wall frame for the polar angles $\theta_{A}$ and $\theta_{B}$ in the $\mathrm{CM}$ frames for the respective $2 \rightarrow 2$ subprocesses (A.31) and (A.32). In this case, the relations are:

$$
\begin{gathered}
-\frac{s_{23}}{s_{13}}=-\frac{(1-y) s_{16}}{(1-x) s_{13}}=\frac{1-c_{A}}{2}=\frac{1-y}{1-x} \frac{1-\cos \theta_{1}}{1+\cos \theta_{1}} \\
-\frac{s_{34}}{s_{35}}=-\frac{y s_{65}}{x s_{35}}=\frac{1-c_{B}}{2}=\frac{y}{x} \frac{1-\cos \theta_{5}}{1+\cos \theta_{5}} .
\end{gathered}
$$

Solving for $\theta_{1}$ and $\theta_{5}$, we have,

$$
\begin{aligned}
\cos \theta_{1} & =\frac{1-y-\frac{1}{2}(1-x)\left(1-c_{A}\right)}{1-y+\frac{1}{2}(1-x)\left(1-c_{A}\right)}, \\
\sin \theta_{1} & =\frac{\sqrt{2(1-x)(1-y)\left(1-c_{A}\right)}}{1-y+\frac{1}{2}(1-x)\left(1-c_{A}\right)}, \\
\cos \theta_{5} & =\frac{y-\frac{1}{2} x\left(1-c_{B}\right)}{y+\frac{1}{2} x\left(1-c_{B}\right)}, \\
\sin \theta_{5} & =\frac{\sqrt{2 x y\left(1-c_{B}\right)}}{y+\frac{1}{2} x\left(1-c_{B}\right)}
\end{aligned}
$$

As in the $2 \rightarrow 4$ case, the dependence of $v$ on the azimuthal angle enters only through $s_{24}$, which appears in $v=s_{23} s_{56} /\left(s_{234} s_{123}\right)$ through $s_{234}=s_{23}+s_{34}+s_{24}$. We compute $s_{24} / s_{36}$ from eq. (A.33) and use eqs. (A.41) and (A.47) to express the result in terms of $x$, $y, \theta_{A}, \theta_{B}$ and $\phi$ :

$$
\frac{s_{24}}{s_{36}}=-\frac{2}{\left(1+c_{A}\right)\left(1+c_{B}\right)}\left[x(1-y)\left(1-c_{A}\right)+y(1-x)\left(1-c_{B}\right)\right.
$$




$$
\left.+2 \sqrt{x y(1-x)(1-y)\left(1-c_{A}\right)\left(1-c_{B}\right)} \cos \phi\right] .
$$

The expression for $v$ in terms of $s_{24} / s_{36}$ for $3 \rightarrow 3$ scattering is,

$$
v=-\frac{1-c_{A}}{1+c_{A}} \frac{1-c_{B}}{1+c_{B}}\left[\frac{s_{24}}{s_{36}}+\frac{1-c_{A}}{1+c_{A}}(1-y)+\frac{1-c_{B}}{1+c_{B}} y\right]^{-1} .
$$

We insert eq. (A.48) for $s_{24} / s_{36}$, and find that

$$
\begin{aligned}
1-\frac{1}{v}=2 \frac{\left(1-c_{A}\right)(1-x)(1-y)+\left(1-c_{B}\right) x y-2 \sqrt{x y(1-x)(1-y)\left(1-c_{A}\right)\left(1-c_{B}\right)} \cos \phi}{\left(1-c_{A}\right)\left(1-c_{B}\right)} \\
=\frac{2}{\left(1-c_{A}\right)\left(1-c_{B}\right)}\left\{\left[\sqrt{\left(1-c_{A}\right)(1-x)(1-y)}-\sqrt{\left(1-c_{B}\right) x y}\right]^{2}\right. \\
\left.+2 \sqrt{x y(1-x)(1-y)\left(1-c_{A}\right)\left(1-c_{B}\right)}(1-\cos \phi)\right\}
\end{aligned}
$$

From eq. (A.50) we can see that for $3 \rightarrow 3$ scattering,

$$
1-\frac{1}{v} \geq 0
$$

which is the complement of the region (A.28) for $2 \rightarrow 4$ scattering. The numerator of eq. (A.50) is minimized, as a function of $\phi$, at $\cos \phi=1$, or $\phi=0$, that is, for a planar scattering configuration. At this minimum, the numerator becomes a perfect square, which can only equal zero, i.e. $v=1$, for

$$
v=1 \quad \Leftrightarrow \quad \phi=0, \quad y=\frac{(1-x)\left(1-c_{A}\right)}{(1-x)\left(1-c_{A}\right)+x\left(1-c_{B}\right)} .
$$

There are two branches that solve the inequality (A.51): $v<0$ and $v \geq 1$. The fact that there are two branches is related to the fact that the $v \rightarrow \infty$ limit corresponds to multiparticle factorization. More generally, as discussed in ref. [61], multi-particle factorization involves taking two cross ratios large at the same rate, with the third cross ratio held fixed.

For $2 \rightarrow 4$ scattering, multi-particle factorization can only occur at the boundary of phase space, where there is a triple-collinear $(1 \rightarrow 3)$ splitting, either in the final state, as shown in figure 11(a), or in the initial state, as depicted in figure 11(b). On the other hand, a multi-particle factorization configuration appears in the middle of the phase space for $3 \rightarrow 3$ scattering, as illustrated in figure 11(c).

This configuration, with $v=\infty$, has

$$
\cos \phi=-\frac{\left(1-c_{A}\right)\left(1-c_{B}\right)-2\left(1-c_{A}\right)(1-x)(1-y)-2\left(1-c_{B}\right) x y}{4 \sqrt{x y(1-x)(1-y)\left(1-c_{A}\right)\left(1-c_{B}\right)}} .
$$

For the MHV configuration studied in this paper, there is no pole in the multi-particle factorization limit, because one cannot factorize an MHV six-point amplitude into two MHV four-point amplitudes. So we should not expect any additional singularity as $v \rightarrow \infty$. Indeed, eqs. (3.28)-(3.33) show that $\mathcal{E}$ has no $\ln v$ singularity in this limit. The Wilson loop $\mathcal{W}^{\text {ns }}$ is also smooth there, since it becomes equal to $\mathcal{E} \exp \left[-\frac{1}{2} \zeta_{2} \gamma_{K}\right]$ as $v \rightarrow \infty$. 


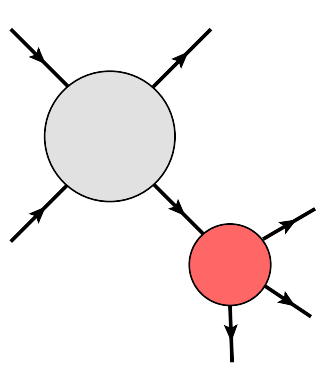

(a)

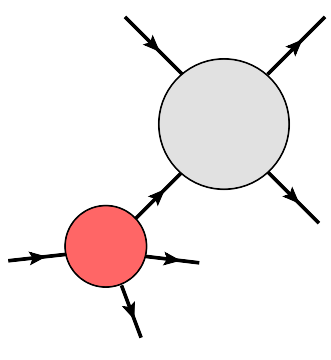

(b)

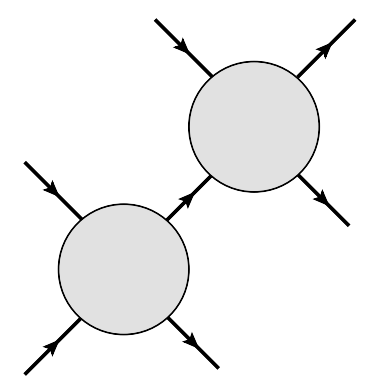

(c)

Figure 11. In $2 \rightarrow 4$ scattering, multi-particle factorization only occurs at the boundary of phase space, where a triple collinear splitting takes place, either in the final state (a), or in the initial state (b). In $3 \rightarrow 3$ scattering, in contrast, multi-particle factorization can appear in the middle of the phase space; both amplitudes in (c) are generic $2 \rightarrow 2$ scattering amplitudes.

\section{B Perturbative expansion of cusp anomalous dimension}

The cusp anomalous dimension is known to all loop orders [72]. Here we give the expansion in the coupling parameter $a$ through 10 loops:

$$
\begin{aligned}
\gamma_{K}(a)=4 & -4 \zeta_{2} a^{2}+22 \zeta_{4} a^{3}-\left(\frac{219}{2} \zeta_{6}+4\left(\zeta_{3}\right)^{2}\right) a^{4}+\left(\frac{1774}{3} \zeta_{8}+8 \zeta_{2}\left(\zeta_{3}\right)^{2}+40 \zeta_{3} \zeta_{5}\right) a^{5} \\
- & \left(\frac{136883}{40} \zeta_{10}+48 \zeta_{4}\left(\zeta_{3}\right)^{2}+80 \zeta_{2} \zeta_{3} \zeta_{5}+102\left(\zeta_{5}\right)^{2}+210 \zeta_{3} \zeta_{7}\right) a^{6} \\
+ & \left(\frac{115201335}{5528} \zeta_{12}+235 \zeta_{6}\left(\zeta_{3}\right)^{2}+4\left(\zeta_{3}\right)^{4}+492 \zeta_{4} \zeta_{3} \zeta_{5}+204 \zeta_{2}\left(\zeta_{5}\right)^{2}+420 \zeta_{2} \zeta_{3} \zeta_{7}\right. \\
& \left.+1092 \zeta_{5} \zeta_{7}+1176 \zeta_{3} \zeta_{9}\right) a^{7} \\
-( & \frac{295221817}{2240} \zeta_{14}+1287 \zeta_{4}\left(\zeta_{5}\right)^{2}+12 \zeta_{2}\left(\zeta_{3}\right)^{4}+2352 \zeta_{2} \zeta_{3} \zeta_{9}+\frac{5955}{2}\left(\zeta_{7}\right)^{2} \\
& +2184 \zeta_{2} \zeta_{5} \zeta_{7}+2472 \zeta_{6} \zeta_{3} \zeta_{5}+2625 \zeta_{4} \zeta_{3} \zeta_{7}+6930 \zeta_{3} \zeta_{11}+6216 \zeta_{5} \zeta_{9}+\frac{2453}{2} \zeta_{8}\left(\zeta_{3}\right)^{2} \\
& \left.+80\left(\zeta_{3}\right)^{3} \zeta_{5}\right) a^{8} \\
+ & \frac{74468151565}{86808} \zeta_{16}+5955\left(\zeta_{7}\right)^{2} \zeta_{2}+84\left(\zeta_{3}\right)^{4} \zeta_{4}+13860 \zeta_{11} \zeta_{2} \zeta_{3}+6633 \zeta_{6}\left(\zeta_{5}\right)^{2} \\
& +37125 \zeta_{5} \zeta_{11}+420 \zeta_{7}\left(\zeta_{3}\right)^{3}+\frac{27537}{4} \zeta_{10}\left(\zeta_{3}\right)^{2}+14022 \zeta_{4} \zeta_{5} \zeta_{7}+240\left(\zeta_{3}\right)^{3} \zeta_{2} \zeta_{5} \\
& +34425 \zeta_{7} \zeta_{9}+\frac{39527}{3} \zeta_{8} \zeta_{3} \zeta_{5}+616\left(\zeta_{5}\right)^{2}\left(\zeta_{3}\right)^{2}+14868 \zeta_{4} \zeta_{3} \zeta_{9}+12432 \zeta_{5} \zeta_{2} \zeta_{9} \\
& \left.+13395 \zeta_{6} \zeta_{3} \zeta_{7}+42471 \zeta_{13} \zeta_{3}\right) a^{9} \\
-( & \frac{40084328285043}{7018720} \zeta_{18}+\frac{311805}{8}\left(\zeta_{7}\right)^{2} \zeta_{4}+\frac{1665675}{8} \zeta_{7} \zeta_{11}+\frac{920205}{4} \zeta_{13} \zeta_{5} \\
& +36062 \zeta_{8}\left(\zeta_{5}\right)^{2}+\frac{28328314}{691} \zeta_{12}\left(\zeta_{3}\right)^{2}+434\left(\zeta_{3}\right)^{4} \zeta_{6}+\frac{2147145}{8} \zeta_{15} \zeta_{3}+2352 \zeta_{9}\left(\zeta_{3}\right)^{3}
\end{aligned}
$$




$$
\begin{aligned}
& +2160\left(\zeta_{5}\right)^{3} \zeta_{3}+\frac{375564}{5} \zeta_{10} \zeta_{3} \zeta_{5}+1704 \zeta_{4}\left(\zeta_{3}\right)^{3} \zeta_{5}+1260\left(\zeta_{3}\right)^{3} \zeta_{7} \zeta_{2} \\
& +1836\left(\zeta_{3}\right)^{2}\left(\zeta_{5}\right)^{2} \zeta_{2}+6594 \zeta_{5}\left(\zeta_{3}\right)^{2} \zeta_{7}+74250 \zeta_{11} \zeta_{2} \zeta_{5}+\frac{176715}{2} \zeta_{11} \zeta_{4} \zeta_{3} \\
& +84942 \zeta_{13} \zeta_{2} \zeta_{3}+68850 \zeta_{7} \zeta_{2} \zeta_{9}+80829 \zeta_{9} \zeta_{4} \zeta_{5}+76671 \zeta_{6} \zeta_{3} \zeta_{9}+\frac{146901}{2} \zeta_{6} \zeta_{5} \zeta_{7} \\
& \left.+72230 \zeta_{8} \zeta_{3} \zeta_{7}+\frac{403165}{4}\left(\zeta_{9}\right)^{2}+4\left(\zeta_{3}\right)^{6}\right) a^{10} \\
& +\mathcal{O}\left(a^{11}\right)
\end{aligned}
$$

\section{Four- and five-loop results for the MHV amplitude in the $3 \rightarrow 3$ self-crossing limit for $v>0$}

In this appendix we give the full four- and five-loop results for the MHV amplitude, normalized by the BDS-like ansatz, $\mathcal{E} \equiv A_{6}^{\mathrm{MHV}} / A_{6}^{\mathrm{BDS}-\text { like }}$, in the $3 \rightarrow 3$ self-crossing limit for $v>0$. We use the same notation used at lower loops in eqs. (3.24), (3.25), (3.26) and (3.27); the argument of the $h_{i}^{[w]}(z)$ is now $z=1 / v$. The four-loop result is

$$
\begin{aligned}
& \mathcal{E}_{3 \rightarrow 3}^{(4)}(v>0)=2 \pi i\left\{-\frac{1}{672} \ln ^{7}|\delta|-\frac{1}{80} \zeta_{2} \ln ^{5}|\delta|-\frac{1}{48} \zeta_{3} \ln ^{4}|\delta|-\frac{7}{24} \zeta_{4} \ln ^{3}|\delta|\right. \\
& +\frac{1}{4}\left(4 \zeta_{5}-3 \zeta_{2} \zeta_{3}\right) \ln ^{2}|\delta|-\frac{1}{48}\left(13 \zeta_{6}+48\left(\zeta_{3}\right)^{2}\right) \ln |\delta| \\
& -\frac{1}{2}\left[120 h_{64}^{[7]}+24 h_{66}^{[7]}+24 h_{68}^{[7]}+3 h_{70}^{[7]}+24 h_{72}^{[7]}+8 h_{74}^{[7]}+4 h_{76}^{[7]}+h_{78}^{[7]}\right. \\
& +24 h_{80}^{[7]}+9 h_{82}^{[7]}+9 h_{84}^{[7]}+h_{86}^{[7]}+6 h_{88}^{[7]}+2 h_{90}^{[7]}+h_{92}^{[7]}+2 h_{94}^{[7]}+6 h_{98}^{[7]} \\
& +6 h_{100}^{[7]}+h_{102}^{[7]}+6 h_{104}^{[7]}+2 h_{106}^{[7]}+h_{108}^{[7]}+2 h_{110}^{[7]}+12 h_{112}^{[7]}+3 h_{114}^{[7]} \\
& +3 h_{116}^{[7]}+2 h_{118}^{[7]}+2 h_{122}^{[7]}+4 h_{124}^{[7]} \\
& +\zeta_{2}\left(-36 h_{16}^{[5]}+3 h_{17}^{[5]}-4 h_{18}^{[5]}+h_{19}^{[5]}-3 h_{20}^{[5]}+h_{21}^{[5]}-h_{22}^{[5]}+2 h_{23}^{[5]}\right. \\
& \left.+6 h_{24}^{[5]}+h_{25}^{[5]}-h_{26}^{[5]}+2 h_{27}^{[5]}-3 h_{28}^{[5]}+2 h_{29}^{[5]}+2 h_{30}^{[5]}\right) \\
& +\zeta_{3}\left(22 h_{8}^{[4]}+11 h_{9}^{[4]}+8 h_{10}^{[4]}+h_{11}^{[4]}+5 h_{12}^{[4]}+h_{13}^{[4]}+h_{14}^{[4]}\right) \\
& -\frac{1}{4} \zeta_{4}\left(39 h_{4}^{[3]}-4 h_{5}^{[3]}-4 h_{6}^{[3]}\right)+\left(14 \zeta_{5}-5 \zeta_{2} \zeta_{3}\right) h_{2}^{[2]} \\
& \left.\left.-\frac{1141}{8} \zeta_{7}+\frac{119}{2} \zeta_{2} \zeta_{5}+\frac{17}{2} \zeta_{3} \zeta_{4}\right]\right\} \\
& -\frac{1}{2}\left\{240 h_{128}^{[8]}+48 h_{130}^{[8]}+48 h_{132}^{[8]}+4 h_{134}^{[8]}+48 h_{136}^{[8]}+16 h_{138}^{[8]}+6 h_{140}^{[8]}+3 h_{142}^{[8]}\right. \\
& +48 h_{144}^{[8]}+16 h_{146}^{[8]}+16 h_{148}^{[8]}+h_{150}^{[8]}+8 h_{152}^{[8]}+3 h_{154}^{[8]}+2 h_{156}^{[8]}+5 h_{158}^{[8]} \\
& +48 h_{160}^{[8]}+18 h_{162}^{[8]}+18 h_{164}^{[8]}+2 h_{166}^{[8]}+18 h_{168}^{[8]}+5 h_{170}^{[8]}+2 h_{172}^{[8]}+5 h_{174}^{[8]} \\
& +12 h_{176}^{[8]}+4 h_{178}^{[8]}+4 h_{180}^{[8]}+4 h_{182}^{[8]}+2 h_{184}^{[8]}+4 h_{186}^{[8]}+4 h_{188}^{[8]}+6 h_{190}^{[8]} \\
& +12 h_{194}^{[8]}+12 h_{196}^{[8]}+2 h_{198}^{[8]}+12 h_{200}^{[8]}+4 h_{202}^{[8]}+2 h_{204}^{[8]}+4 h_{206}^{[8]} \\
& +12 h_{208}^{[8]}+4 h_{210}^{[8]}+4 h_{212}^{[8]}+4 h_{214}^{[8]}+2 h_{216}^{[8]}+4 h_{218}^{[8]}+4 h_{220}^{[8]}+6 h_{222}^{[8]} \\
& +24 h_{224}^{[8]}+6 h_{226}^{[8]}+6 h_{228}^{[8]}+4 h_{230}^{[8]}+6 h_{232}^{[8]}+5 h_{234}^{[8]}+5 h_{238}^{[8]}+4 h_{236}^{[8]} \\
& +4 h_{242}^{[8]}+4 h_{244}^{[8]}+5 h_{246}^{[8]}+8 h_{248}^{[8]}+5 h_{250}^{[8]}+30 h_{254}^{[8]}
\end{aligned}
$$




$$
\begin{aligned}
& +\zeta_{2}\left(-552 h_{32}^{[6]}+4 h_{33}^{[6]}-104 h_{34}^{[6]}+3 h_{35}^{[6]}-104 h_{36}^{[6]}+h_{37}^{[6]}-17 h_{38}^{[6]}+5 h_{39}^{[6]}\right. \\
& \quad-102 h_{40}^{[6]}+2 h_{41}^{[6]}-40 h_{42}^{[6]}+5 h_{43}^{[6]}-26 h_{44}^{[6]}+4 h_{45}^{[6]}-h_{46}^{[6]}+6 h_{47}^{[6]} \\
& \quad+12 h_{48}^{[6]}+2 h_{49}^{[6]}-26 h_{50}^{[6]}+4 h_{51}^{[6]}-26 h_{52}^{[6]}+4 h_{53}^{[6]}-h_{54}^{[6]}+6 h_{55}^{[6]} \\
& \left.\quad-54 h_{56}^{[6]}+4 h_{57}^{[6]}-10 h_{58}^{[6]}+5 h_{59}^{[6]}+4 h_{60}^{[6]}+5 h_{61}^{[6]}-15 h_{62}^{[6]}+30 h_{63}^{[6]}\right) \\
& +\zeta_{3}\left(44 h_{16}^{[5]}+23 h_{17}^{[5]}+15 h_{18}^{[5]}-h_{19}^{[5]}+16 h_{20}^{[5]}+3 h_{21}^{[5]}-2 h_{23}^{[5]}\right. \\
& \left.\quad+10 h_{24}^{[5]}+2 h_{25}^{[5]}-2 h_{27}^{[5]}+2 h_{28}^{[5]}+h_{29}^{[5]}-h_{30}^{[5]}-20 h_{31}^{[5]}\right) \\
& -\frac{1}{4} \zeta_{4}\left(558 h_{8}^{[4]}+142 h_{9}^{[4]}+267 h_{10}^{[4]}+22 h_{11}^{[4]}+232 h_{12}^{[4]}+22 h_{13}^{[4]}+67 h_{14}^{[4]}+58 h_{15}^{[4]}\right) \\
& +\zeta_{5}\left(28 h_{4}^{[3]}+21 h_{5}^{[3]}+8 h_{6}^{[3]}-16 h_{7}^{[3]}\right)-\zeta_{2} \zeta_{3}\left(98 h_{4}^{[3]}+54 h_{5}^{[3]}+25 h_{6}^{[3]}+10 h_{7}^{[3]}\right) \\
& -\frac{1}{48} \zeta_{6}\left(8477 h_{2}^{[2]}+3687 h_{3}^{[2]}\right)+\frac{3}{2}\left(\zeta_{3}\right)^{2}\left(4 h_{2}^{[2]}+h_{3}^{[2]}\right) \\
& \left.+\frac{1}{2}\left(26 \zeta_{7}-228 \zeta_{2} \zeta_{5}-175 \zeta_{3} \zeta_{4}\right) h_{1}^{[1]}-\frac{5}{2} \zeta_{5,3}-\frac{56911}{72} \zeta_{8}+\frac{63}{2} \zeta_{3} \zeta_{5}-36 \zeta_{2}\left(\zeta_{3}\right)^{2}\right\}, \quad(\mathrm{C} .1)
\end{aligned}
$$

and the five-loop one is

$$
\begin{aligned}
& \mathcal{E}_{3 \rightarrow 3}^{(5)}(v>0)=2 \pi i\left\{\frac{1}{6912} \ln ^{9}|\delta|+\frac{1}{336} \zeta_{2} \ln ^{7}|\delta|+\frac{5}{288} \zeta_{3} \ln ^{6}|\delta|+\frac{9}{80} \zeta_{4} \ln ^{5}|\delta|\right. \\
& +\frac{1}{24}\left(6 \zeta_{5}+7 \zeta_{2} \zeta_{3}\right) \ln ^{4}|\delta|+\frac{1}{72}\left(115 \zeta_{6}+48\left(\zeta_{3}\right)^{2}\right) \ln ^{3}|\delta| \\
& +\frac{1}{16}\left(-55 \zeta_{7}+68 \zeta_{2} \zeta_{5}+44 \zeta_{3} \zeta_{4}\right) \ln ^{2}|\delta| \\
& +\frac{1}{72}\left(257 \zeta_{8}+810 \zeta_{3} \zeta_{5}+18 \zeta_{2}\left(\zeta_{3}\right)^{2}\right) \ln |\delta| \\
& +\frac{1}{4}\left[3360 h_{256}^{[9]}+720 h_{258}^{[9]}+720 h_{260}^{[9]}+20 h_{262}^{[9]}\right. \\
& +720 h_{264}^{[9]}+192 h_{266}^{[9]}+32 h_{268}^{[9]}+20 h_{270}^{[9]}+720 h_{272}^{[9]}+196 h_{274}^{[9]} \\
& +196 h_{276}^{[9]}+9 h_{278}^{[9]}+48 h_{280}^{[9]}+21 h_{282}^{[9]}+16 h_{284}^{[9]}+11 h_{286}^{[9]}+720 h_{288}^{[9]} \\
& +204 h_{290}^{[9]}+204 h_{292}^{[9]}+10 h_{294}^{[9]}+204 h_{296}^{[9]}+56 h_{298}^{[9]}+10 h_{300}^{[9]}+15 h_{302}^{[9]} \\
& +72 h_{304}^{[9]}+26 h_{306}^{[9]}+26 h_{308}^{[9]}+11 h_{310}^{[9]}+12 h_{312}^{[9]}+11 h_{314}^{[9]}+14 h_{316}^{[9]} \\
& +6 h_{318}^{[9]}+720 h_{320}^{[9]}+228 h_{322}^{[9]}+228 h_{324}^{[9]}+16 h_{326}^{[9]}+228 h_{328}^{[9]}+66 h_{330}^{[9]} \\
& +16 h_{332}^{[9]}+18 h_{334}^{[9]}+228 h_{336}^{[9]}+66 h_{338}^{[9]}+66 h_{340}^{[9]}+17 h_{342}^{[9]}+16 h_{344}^{[9]} \\
& +18 h_{346}^{[9]}+18 h_{348}^{[9]}+10 h_{350}^{[9]}+120 h_{352}^{[9]}+40 h_{354}^{[9]}+40 h_{356}^{[9]}+16 h_{358}^{[9]} \\
& +40 h_{360}^{[9]}+22 h_{362}^{[9]}+14 h_{364}^{[9]}+11 h_{366}^{[9]}+12 h_{368}^{[9]}+14 h_{370}^{[9]}+14 h_{372}^{[9]} \\
& +11 h_{374}^{[9]}+20 h_{376}^{[9]}+13 h_{378}^{[9]}+6 h_{380}^{[9]}+18 h_{382}^{[9]}+120 h_{386}^{[9]}+120 h_{388}^{[9]} \\
& +19 h_{390}^{[9]}+120 h_{392}^{[9]}+43 h_{394}^{[9]}+18 h_{396}^{[9]}+20 h_{398}^{[9]}+120 h_{400}^{[9]}+42 h_{402}^{[9]} \\
& +42 h_{404}^{[9]}+18 h_{406}^{[9]}+16 h_{408}^{[9]}+19 h_{410}^{[9]}+20 h_{412}^{[9]}+10 h_{414}^{[9]}+120 h_{416}^{[9]}+40 h_{418}^{[9]}+40 h_{420}^{[9]} \\
& +16 h_{422}^{[9]}+40 h_{424}^{[9]}+22 h_{426}^{[9]}+14 h_{428}^{[9]}+11 h_{430}^{[9]}+12 h_{432}^{[9]}+14 h_{434}^{[9]}+14 h_{436}^{[9]}+11 h_{438}^{[9]} \\
& +20 h_{440}^{[9]}+13 h_{442}^{[9]}+6 h_{444}^{[9]}+18 h_{446}^{[9]}+240 h_{448}^{[9]}+60 h_{450}^{[9]}+60 h_{452}^{[9]}+14 h_{454}^{[9]}+60 h_{456}^{[9]} \\
& +24 h_{458}^{[9]}+16 h_{460}^{[9]}+8 h_{462}^{[9]}+60 h_{464}^{[9]}+26 h_{466}^{[9]}+26 h_{468}^{[9]}+12 h_{470}^{[9]}+20 h_{472}^{[9]}+14 h_{474}^{[9]} \\
& +8 h_{476}^{[9]}+18 h_{478}^{[9]}+20 h_{482}^{[9]}+20 h_{484}^{[9]}+12 h_{486}^{[9]}+20 h_{488}^{[9]}+14 h_{490}^{[9]}+8 h_{492}^{[9]}+18 h_{494}^{[9]} \\
& +24 h_{496}^{[9]}+8 h_{498}^{[9]}+8 h_{500}^{[9]}+18 h_{502}^{[9]}+16 h_{504}^{[9]}+22 h_{506}^{[9]}+20 h_{508}^{[9]} \\
& +\zeta_{2}\left(-960 h_{64}^{[7]}+20 h_{65}^{[7]}-168 h_{66}^{[7]}+20 h_{67}^{[7]}-164 h_{68}^{[7]}+9 h_{69}^{[7]}-3 h_{70}^{[7]}+11 h_{71}^{[7]}-156 h_{72}^{[7]}\right. \\
& +10 h_{73}^{[7]}-46 h_{74}^{[7]}+15 h_{75}^{[7]}-10 h_{76}^{[7]}+11 h_{77}^{[7]}+5 h_{78}^{[7]}+6 h_{79}^{[7]}-132 h_{80}^{[7]}+16 h_{81}^{[7]}-48 h_{82}^{[7]} \\
& +18 h_{83}^{[7]}-48 h_{84}^{[7]}+17 h_{85}^{[7]}+10 h_{86}^{[7]}+10 h_{87}^{[7]}-20 h_{88}^{[7]}+16 h_{89}^{[7]}+2 h_{90}^{[7]}+11 h_{91}^{[7]}+8 h_{92}^{[7]}
\end{aligned}
$$




$$
\begin{aligned}
& +11 h_{93}^{[7]}+3 h_{94}^{[7]}+18 h_{95}^{[7]}+120 h_{96}^{[7]}+19 h_{97}^{[7]}-17 h_{98}^{[7]}+20 h_{99}^{[7]}-18 h_{100}^{[7]}+18 h_{101}^{[7]}+11 h_{102}^{[7]} \\
& +10 h_{103}^{[7]}-20 h_{104}^{[7]}+16 h_{105}^{[7]}+2 h_{106}^{[7]}+11 h_{107}^{[7]}+8 h_{108}^{[7]}+11 h_{109}^{[7]}+3 h_{110}^{[7]}+18 h_{111}^{[7]} \\
& -60 h_{112}^{[7]}+14 h_{113}^{[7]}-6 h_{114}^{[7]}+8 h_{115}^{[7]}-4 h_{116}^{[7]}+12 h_{117}^{[7]}+4 h_{118}^{[7]}+18 h_{119}^{[7]} \\
& \left.+20 h_{120}^{[7]}+12 h_{121}^{[7]}+4 h_{122}^{[7]}+18 h_{123}^{[7]}-4 h_{124}^{[7]}+18 h_{125}^{[7]}+14 h_{126}^{[7]}\right) \\
& +\zeta_{3}\left(760 h_{32}^{[6]}+332 h_{33}^{[6]}+200 h_{34}^{[6]}+15 h_{35}^{[6]}+208 h_{36}^{[6]}+87 h_{37}^{[6]}+14 h_{38}^{[6]}+2 h_{39}^{[6]}+228 h_{40}^{[6]}\right. \\
& +98 h_{41}^{[6]}+55 h_{42}^{[6]}+8 h_{43}^{[6]}+24 h_{44}^{[6]}+19 h_{45}^{[6]}+5 h_{46}^{[6]}+2 h_{47}^{[6]}+108 h_{48}^{[6]}+48 h_{49}^{[6]}+26 h_{50}^{[6]} \\
& +8 h_{51}^{[6]}+24 h_{52}^{[6]}+19 h_{53}^{[6]}+5 h_{54}^{[6]}+2 h_{55}^{[6]}+44 h_{56}^{[6]}+24 h_{57}^{[6]}+17 h_{58}^{[6]}+2 h_{59}^{[6]}+4 h_{60}^{[6]} \\
& \left.+2 h_{61}^{[6]}-2 h_{62}^{[6]}+24 h_{63}^{[6]}\right) \\
& +\frac{1}{4} \zeta_{4}\left(-1380 h_{16}^{[5]}+30 h_{17}^{[5]}-296 h_{18}^{[5]}+4 h_{19}^{[5]}-302 h_{20}^{[5]}+11 h_{21}^{[5]}-28 h_{22}^{[5]}+42 h_{23}^{[5]}+12 h_{24}^{[5]}\right. \\
& \left.+11 h_{25}^{[5]}-28 h_{26}^{[5]}+42 h_{27}^{[5]}-74 h_{28}^{[5]}+42 h_{29}^{[5]}+42 h_{30}^{[5]}-20 h_{31}^{[5]}\right) \\
& +\frac{1}{2} \zeta_{5}\left(1104 h_{8}^{[4]}+516 h_{9}^{[4]}+355 h_{10}^{[4]}+76 h_{11}^{[4]}+194 h_{12}^{[4]}+76 h_{13}^{[4]}+56 h_{14}^{[4]}+56 h_{15}^{[4]}\right) \\
& -\zeta_{2} \zeta_{3}\left(200 h_{8}^{[4]}+87 h_{9}^{[4]}+62 h_{10}^{[4]}+10 h_{11}^{[4]}+32 h_{12}^{[4]}+10 h_{13}^{[4]}+9 h_{14}^{[4]}-4 h_{15}^{[4]}\right) \\
& -\frac{1}{48} \zeta_{6}\left(10540 h_{4}^{[3]}-75\left(h_{5}^{[3]}+h_{6}^{[3]}\right)+372 h_{7}^{[3]}\right)+\frac{1}{2}\left(\zeta_{3}\right)^{2}\left(196 h_{4}^{[3]}+37 h_{5}^{[3]}+21 h_{6}^{[3]}-8 h_{7}^{[3]}\right) \\
& +\frac{1}{16} \zeta_{7}\left(6280 h_{2}^{[2]}+183 h_{3}^{[2]}\right)-\frac{1}{2} \zeta_{2} \zeta_{5}\left(284 h_{2}^{[2]}-5 h_{3}^{[2]}\right)-\frac{1}{4} \zeta_{3} \zeta_{4}\left(355 h_{2}^{[2]}-22 h_{3}^{[2]}\right) \\
& -\frac{1}{2}\left(\zeta_{5,3}+\frac{217}{24} \zeta_{8}+15 \zeta_{2}\left(\zeta_{3}\right)^{2}-17 \zeta_{3} \zeta_{5}\right) h_{1}^{[1]} \\
& \left.\left.-\frac{40369}{16} \zeta_{9}+\frac{7645}{8} \zeta_{2} \zeta_{7}+\frac{3119}{16} \zeta_{3} \zeta_{6}+\frac{2295}{8} \zeta_{4} \zeta_{5}-15\left(\zeta_{3}\right)^{3}\right]\right\} \\
& +\frac{1}{8}\left\{13440 h_{512}^{[10]}+2880 h_{514}^{[10]}+2880 h_{516}^{[10]}-160 h_{518}^{[10]}+2880 h_{520}^{[10]}+768 h_{522}^{[10]}+80 h_{524}^{[10]}\right. \\
& +152 h_{526}^{[10]}+2880 h_{528}^{[10]}+768 h_{530}^{[10]}+768 h_{532}^{[10]}-24 h_{534}^{[10]}+128 h_{536}^{[10]}+72 h_{538}^{[10]}+80 h_{540}^{[10]} \\
& +48 h_{542}^{[10]}+2880 h_{544}^{[10]}+784 h_{546}^{[10]}+784 h_{548}^{[10]}-16 h_{550}^{[10]}+784 h_{552}^{[10]}+212 h_{554}^{[10]}+36 h_{556}^{[10]} \\
& +80 h_{558}^{[10]}+192 h_{560}^{[10]}+84 h_{562}^{[10]}+84 h_{564}^{[10]}+44 h_{566}^{[10]}+64 h_{568}^{[10]}+44 h_{570}^{[10]}+44 h_{572}^{[10]}+17 h_{574}^{[10]} \\
& +2880 h_{576}^{[10]}+816 h_{578}^{[10]}+816 h_{580}^{[10]}-16 h_{582}^{[10]}+816 h_{584}^{[10]}+224 h_{586}^{[10]}+40 h_{588}^{[10]}+74 h_{590}^{[10]} \\
& +816 h_{592}^{[10]}+224 h_{594}^{[10]}+224 h_{596}^{[10]}+34 h_{598}^{[10]}+40 h_{600}^{[10]}+50 h_{602}^{[10]}+60 h_{604}^{[10]}+21 h_{606}^{[10]}+288 h_{608}^{[10]} \\
& +104 h_{610}^{[10]}+104 h_{612}^{[10]}+48 h_{614}^{[10]}+104 h_{616}^{[10]}+58 h_{618}^{[10]}+44 h_{620}^{[10]}+29 h_{622}^{[10]}+48 h_{624}^{[10]}+44 h_{626}^{[10]} \\
& +44 h_{628}^{[10]}+30 h_{630}^{[10]}+56 h_{632}^{[10]}+34 h_{634}^{[10]}+24 h_{636}^{[10]}+80 h_{638}^{[10]}+2880 h_{640}^{[10]}+912 h_{642}^{[10]}+912 h_{644}^{[10]} \\
& +912 h_{648}^{[10]}+264 h_{650}^{[10]}+64 h_{652}^{[10]}+88 h_{654}^{[10]}+912 h_{656}^{[10]}+264 h_{658}^{[10]}+264 h_{660}^{[10]}+44 h_{662}^{[10]}+64 h_{664}^{[10]} \\
& +64 h_{666}^{[10]}+72 h_{668}^{[10]}+36 h_{670}^{[10]}+912 h_{672}^{[10]}+264 h_{674}^{[10]}+264 h_{676}^{[10]}+44 h_{678}^{[10]}+264 h_{680}^{[10]}+118 h_{682}^{[10]} \\
& +68 h_{684}^{[10]}+50 h_{686}^{[10]}+64 h_{688}^{[10]}+72 h_{690}^{[10]}+72 h_{692}^{[10]}+46 h_{694}^{[10]}+72 h_{696}^{[10]}+50 h_{698}^{[10]}+40 h_{700}^{[10]} \\
& +84 h_{702}^{[10]}+480 h_{704}^{[10]}+160 h_{706}^{[10]}+160 h_{708}^{[10]}+64 h_{710}^{[10]}+160 h_{712}^{[10]}+88 h_{714}^{[10]}+64 h_{716}^{[10]}+42 h_{718}^{[10]} \\
& +160 h_{720}^{[10]}+88 h_{722}^{[10]}+88 h_{724}^{[10]}+48 h_{726}^{[10]}+56 h_{728}^{[10]}+50 h_{730}^{[10]}+44 h_{732}^{[10]}+81 h_{734}^{[10]}+48 h_{736}^{[10]} \\
& +56 h_{738}^{[10]}+56 h_{740}^{[10]}+36 h_{742}^{[10]}+56 h_{744}^{[10]}+50 h_{746}^{[10]}+44 h_{748}^{[10]}+80 h_{750}^{[10]}+80 h_{752}^{[10]}+52 h_{754}^{[10]} \\
& +52 h_{756}^{[10]}+78 h_{758}^{[10]}+24 h_{760}^{[10]}+78 h_{762}^{[10]}+72 h_{764}^{[10]}+180 h_{766}^{[10]}+480 h_{770}^{[10]}+480 h_{772}^{[10]}+48 h_{774}^{[10]} \\
& +480 h_{776}^{[10]}+172 h_{778}^{[10]}+76 h_{780}^{[10]}+86 h_{782}^{[10]}+480 h_{784}^{[10]}+172 h_{786}^{[10]}+172 h_{788}^{[10]}+66 h_{790}^{[10]}+72 h_{792}^{[10]} \\
& +78 h_{794}^{[10]}+80 h_{796}^{[10]}+38 h_{798}^{[10]}+480 h_{800}^{[10]}+168 h_{802}^{[10]}+168 h_{804}^{[10]}+64 h_{806}^{[10]}+168 h_{808}^{[10]}+98 h_{810}^{[10]} \\
& +72 h_{812}^{[10]}+44 h_{814}^{[10]}+64 h_{816}^{[10]}+76 h_{818}^{[10]}+76 h_{820}^{[10]}+44 h_{822}^{[10]}+80 h_{824}^{[10]}+52 h_{826}^{[10]}+40 h_{828}^{[10]} \\
& +62 h_{830}^{[10]}+480 h_{832}^{[10]}+160 h_{834}^{[10]}+160 h_{836}^{[10]}+64 h_{838}^{[10]}+160 h_{840}^{[10]}+88 h_{842}^{[10]}+64 h_{844}^{[10]}+42 h_{846}^{[10]} \\
& +160 h_{848}^{[10]}+88 h_{850}^{[10]}+88 h_{852}^{[10]}+48 h_{854}^{[10]}+56 h_{856}^{[10]}+50 h_{858}^{[10]}+44 h_{860}^{[10]}+81 h_{862}^{[10]}+48 h_{864}^{[10]} \\
& +56 h_{866}^{[10]}+56 h_{868}^{[10]}+36 h_{870}^{[10]}+56 h_{872}^{[10]}+50 h_{874}^{[10]}+44 h_{876}^{[10]}+80 h_{878}^{[10]}+80 h_{880}^{[10]}+52 h_{882}^{[10]}
\end{aligned}
$$




$$
\begin{aligned}
& +52 h_{884}^{[10]}+78 h_{886}^{[10]}+24 h_{888}^{[10]}+78 h_{890}^{[10]}+72 h_{892}^{[10]}+180 h_{894}^{[10]}+960 h_{896}^{[10]}+240 h_{898}^{[10]}+240 h_{900}^{[10]} \\
& +64 h_{902}^{[10]}+240 h_{904}^{[10]}+96 h_{906}^{[10]}+56 h_{908}^{[10]}+32 h_{910}^{[10]}+240 h_{912}^{[10]}+96 h_{914}^{[10]}+96 h_{916}^{[10]}+32 h_{918}^{[10]} \\
& +64 h_{920}^{[10]}+36 h_{922}^{[10]}+32 h_{924}^{[10]}+50 h_{926}^{[10]}+240 h_{928}^{[10]}+104 h_{930}^{[10]}+104 h_{932}^{[10]}+36 h_{934}^{[10]}+104 h_{936}^{[10]} \\
& +62 h_{938}^{[10]}+48 h_{940}^{[10]}+79 h_{942}^{[10]}+80 h_{944}^{[10]}+56 h_{946}^{[10]}+56 h_{948}^{[10]}+76 h_{950}^{[10]}+32 h_{952}^{[10]}+76 h_{954}^{[10]} \\
& +72 h_{956}^{[10]}+150 h_{958}^{[10]}+80 h_{962}^{[10]}+80 h_{964}^{[10]}+64 h_{966}^{[10]}+80 h_{968}^{[10]}+56 h_{970}^{[10]}+48 h_{972}^{[10]}+64 h_{974}^{[10]} \\
& +80 h_{976}^{[10]}+56 h_{978}^{[10]}+56 h_{980}^{[10]}+84 h_{982}^{[10]}+32 h_{984}^{[10]}+76 h_{986}^{[10]}+72 h_{988}^{[10]}+144 h_{990}^{[10]}+96 h_{992}^{[10]} \\
& +32 h_{994}^{[10]}+32 h_{996}^{[10]}+40 h_{998}^{[10]}+32 h_{1000}^{[10]}+76 h_{1002}^{[10]}+72 h_{1004}^{[10]}+140 h_{1006}^{[10]}+64 h_{1008}^{[10]}+88 h_{1010}^{[10]} \\
& +88 h_{1012}^{[10]}+140 h_{1014}^{[10]}+80 h_{1016}^{[10]}+140 h_{1018}^{[10]}+840 h_{1022}^{[10]} \\
& +\zeta_{2}\left(-30720 h_{128}^{[8]}-160 h_{129}^{[8]}-6432 h_{130}^{[8]}+152 h_{131}^{[8]}-6432 h_{132}^{[8]}-24 h_{133}^{[8]}-248 h_{134}^{[8]}\right. \\
& +48 h_{135}^{[8]}-6416 h_{136}^{[8]}-16 h_{137}^{[8]}-1748 h_{138}^{[8]}+80 h_{139}^{[8]}-396 h_{140}^{[8]}+44 h_{141}^{[8]}-116 h_{142}^{[8]} \\
& +17 h_{143}^{[8]}-6384 h_{144}^{[8]}-16 h_{145}^{[8]}-1816 h_{146}^{[8]}+74 h_{147}^{[8]}-1816 h_{148}^{[8]}+34 h_{149}^{[8]}-50 h_{150}^{[8]} \\
& +21 h_{151}^{[8]}-616 h_{152}^{[8]}+48 h_{153}^{[8]}-202 h_{154}^{[8]}+29 h_{155}^{[8]}-76 h_{156}^{[8]}+30 h_{157}^{[8]}-106 h_{158}^{[8]} \\
& +80 h_{159}^{[8]}-6288 h_{160}^{[8]}-2016 h_{162}^{[8]}+88 h_{163}^{[8]}-2016 h_{164}^{[8]}+44 h_{165}^{[8]}-96 h_{166}^{[8]}+36 h_{167}^{[8]} \\
& -2016 h_{168}^{[8]}+44 h_{169}^{[8]}-542 h_{170}^{[8]}+50 h_{171}^{[8]}-88 h_{172}^{[8]}+46 h_{173}^{[8]}-130 h_{174}^{[8]}+84 h_{175}^{[8]} \\
& -1040 h_{176}^{[8]}+64 h_{177}^{[8]}-312 h_{178}^{[8]}+42 h_{179}^{[8]}-312 h_{180}^{[8]}-90 h_{182}^{[8]}+48 h_{181}^{[8]}+81 h_{183}^{[8]} \\
& -64 h_{184}^{[8]}+36 h_{185}^{[8]}-90 h_{186}^{[8]}+80 h_{187}^{[8]}-148 h_{188}^{[8]}+78 h_{189}^{[8]}+18 h_{190}^{[8]}+180 h_{191}^{[8]} \\
& +480 h_{192}^{[8]}+48 h_{193}^{[8]}-1028 h_{194}^{[8]}+86 h_{195}^{[8]}-1028 h_{196}^{[8]}+66 h_{197}^{[8]}-102 h_{198}^{[8]}+38 h_{199}^{[8]} \\
& -1032 h_{200}^{[8]}+64 h_{201}^{[8]}-322 h_{202}^{[8]}+44 h_{203}^{[8]}-84 h_{204}^{[8]}+44 h_{205}^{[8]}-148 h_{206}^{[8]}+62 h_{207}^{[8]} \\
& -1040 h_{208}^{[8]}+64 h_{209}^{[8]}-312 h_{210}^{[8]}+42 h_{211}^{[8]}-312 h_{212}^{[8]}+48 h_{213}^{[8]}-90 h_{214}^{[8]}+81 h_{215}^{[8]} \\
& -64 h_{216}^{[8]}+36 h_{217}^{[8]}-90 h_{218}^{[8]}+80 h_{219}^{[8]}-148 h_{220}^{[8]}+78 h_{221}^{[8]}+18 h_{222}^{[8]}+180 h_{223}^{[8]} \\
& -2160 h_{224}^{[8]}+64 h_{225}^{[8]}-504 h_{226}^{[8]}+32 h_{227}^{[8]}-504 h_{228}^{[8]}+32 h_{229}^{[8]}-124 h_{230}^{[8]}+50 h_{231}^{[8]} \\
& -496 h_{232}^{[8]}+36 h_{233}^{[8]}-198 h_{234}^{[8]}+79 h_{235}^{[8]}-144 h_{236}^{[8]}+76 h_{237}^{[8]}-4 h_{238}^{[8]}+150 h_{239}^{[8]} \\
& +80 h_{240}^{[8]}+64 h_{241}^{[8]}-144 h_{242}^{[8]}+64 h_{243}^{[8]}-144 h_{244}^{[8]}+84 h_{245}^{[8]}-4 h_{246}^{[8]}+144 h_{247}^{[8]} \\
& \left.-208 h_{248}^{[8]}+40 h_{249}^{[8]}-4 h_{250}^{[8]}+140 h_{251}^{[8]}-72 h_{252}^{[8]}+140 h_{253}^{[8]}-60 h_{254}^{[8]}+840 h_{255}^{[8]}\right) \\
& +\zeta_{3}\left(3040 h_{64}^{[7]}+1304 h_{65}^{[7]}+792 h_{66}^{[7]}+16 h_{67}^{[7]}+800 h_{68}^{[7]}+308 h_{69}^{[7]}+40 h_{70}^{[7]}+27 h_{71}^{[7]}\right. \\
& +832 h_{72}^{[7]}+334 h_{73}^{[7]}+190 h_{74}^{[7]}+19 h_{75}^{[7]}+56 h_{76}^{[7]}+43 h_{77}^{[7]}+14 h_{78}^{[7]}-36 h_{79}^{[7]} \\
& +912 h_{80}^{[7]}+376 h_{81}^{[7]}+220 h_{82}^{[7]}+20 h_{83}^{[7]}+220 h_{84}^{[7]}+118 h_{85}^{[7]}+26 h_{86}^{[7]}-24 h_{87}^{[7]} \\
& +96 h_{88}^{[7]}+70 h_{89}^{[7]}+40 h_{90}^{[7]}-25 h_{91}^{[7]}+20 h_{92}^{[7]}-24 h_{93}^{[7]}-26 h_{94}^{[7]}-96 h_{95}^{[7]} \\
& +432 h_{96}^{[7]}+182 h_{97}^{[7]}+106 h_{98}^{[7]}+38 h_{99}^{[7]}+104 h_{100}^{[7]}+80 h_{101}^{[7]}+32 h_{102}^{[7]}+2 h_{103}^{[7]} \\
& +96 h_{104}^{[7]}+70 h_{105}^{[7]}+40 h_{106}^{[7]}-25 h_{107}^{[7]}+20 h_{108}^{[7]}-24 h_{109}^{[7]}-26 h_{110}^{[7]}-96 h_{111}^{[7]} \\
& +176 h_{112}^{[7]}+104 h_{113}^{[7]}+64 h_{114}^{[7]}-10 h_{115}^{[7]}+68 h_{116}^{[7]}-3 h_{117}^{[7]}-20 h_{118}^{[7]}-70 h_{119}^{[7]} \\
& \left.+16 h_{120}^{[7]}-28 h_{122}^{[7]}-64 h_{123}^{[7]}-8 h_{124}^{[7]}-60 h_{125}^{[7]}-52 h_{126}^{[7]}-560 h_{127}^{[7]}\right) \\
& -\zeta_{4}\left(9060 h_{32}^{[6]}+520 h_{33}^{[6]}+2766 h_{34}^{[6]}+330 h_{35}^{[6]}+2936 h_{36}^{[6]}+179 h_{37}^{[6]}+\frac{785}{2} h_{38}^{[6]}+220 h_{39}^{[6]}\right. \\
& +3422 h_{40}^{[6]}+289 h_{41}^{[6]}+\frac{1899}{2} h_{42}^{[6]}+304 h_{43}^{[6]}+588 h_{44}^{[6]}+\frac{449}{2} h_{45}^{[6]}+\frac{405}{2} h_{46}^{[6]}-33 h_{47}^{[6]} \\
& +2388 h_{48}^{[6]}+344 h_{49}^{[6]}+\frac{1261}{2} h_{50}^{[6]}+364 h_{51}^{[6]}+588 h_{52}^{[6]}+\frac{449}{2} h_{53}^{[6]}+\frac{405}{2} h_{54}^{[6]}-33 h_{55}^{[6]} \\
& \left.+794 h_{56}^{[6]}+291 h_{57}^{[6]}+\frac{735}{2} h_{58}^{[6]}+38 h_{59}^{[6]}+358 h_{60}^{[6]}+54 h_{61}^{[6]}+15 h_{62}^{[6]}-350 h_{63}^{[6]}\right) \\
& +\zeta_{5}\left(2208 h_{16}^{[5]}+1097 h_{17}^{[5]}+657 h_{18}^{[5]}+33 h_{19}^{[5]}+710 h_{20}^{[5]}+266 h_{21}^{[5]}+50 h_{22}^{[5]}-30 h_{23}^{[5]}\right. \\
& \left.+388 h_{24}^{[5]}+138 h_{25}^{[5]}+50 h_{26}^{[5]}-30 h_{27}^{[5]}+112 h_{28}^{[5]}+57 h_{29}^{[5]}-448 h_{31}^{[5]}\right)
\end{aligned}
$$




$$
\begin{aligned}
& -\zeta_{2} \zeta_{3}\left(6880 h_{16}^{[5]}+2940 h_{17}^{[5]}+1896 h_{18}^{[5]}+227 h_{19}^{[5]}+2072 h_{20}^{[5]}+856 h_{21}^{[5]}+212 h_{22}^{[5]}+70 h_{23}^{[5]}\right. \\
& \left.\quad+992 h_{24}^{[5]}+416 h_{25}^{[5]}+212 h_{26}^{[5]}+70 h_{27}^{[5]}+388 h_{28}^{[5]}+259 h_{29}^{[5]}+68 h_{30}^{[5]}-24 h_{31}^{[5]}\right) \\
& +\zeta_{6}\left(-\frac{28465}{3} h_{8}^{[4]}-\frac{3819}{4} h_{9}^{[4]}-\frac{23921}{8} h_{10}^{[4]}-\frac{2239}{24} h_{11}^{[4]}-\frac{6863}{4} h_{12}^{[4]}-\frac{2239}{24} h_{13}^{[4]}\right. \\
& \left.\quad-\frac{19765}{24} h_{14}^{[4]}-\frac{5}{12} h_{15}^{[4]}\right) \\
& +\left(\zeta_{3}\right)^{2}\left(392 h_{8}^{[4]}+\frac{103}{2} h_{9}^{[4]}+111 h_{10}^{[4]}+\frac{41}{2} h_{11}^{[4]}+42 h_{12}^{[4]}+\frac{41}{2} h_{13}^{[4]}+28 h_{14}^{[4]}+34 h_{15}^{[4]}\right) \\
& +\zeta_{7}\left(1570 h_{4}^{[3]}+\frac{6959}{8} h_{5}^{[3]}+\frac{2319}{8} h_{6}^{[3]}-\frac{3777}{16} h_{7}^{[3]}\right) \\
& -\zeta_{2} \zeta_{5}\left(4984 h_{4}^{[3]}+2367 h_{5}^{[3]}+921 h_{6}^{[3]}+\frac{659}{2} h_{7}^{[3]}\right) \\
& -\zeta_{3} \zeta_{4}\left(2435 h_{4}^{[3]}+1056 h_{5}^{[3]}+\frac{601}{2} h_{6}^{[3]}+647 h_{7}^{[3]}\right)-\frac{9}{10} \zeta_{5,3} h_{3}^{[2]} \\
& -\zeta_{8}\left(\frac{2461055}{288} h_{2}^{[2]}+\frac{4148393}{1440} h_{3}^{[2]}\right)-\zeta_{2}\left(\zeta_{3}\right)^{2}\left(886 h_{2}^{[2]}+165 h_{3}^{[2]}\right)+\zeta_{3} \zeta_{5}\left(578 h_{2}^{[2]}+120 h_{3}^{[2]}\right) \\
& +\left(\frac{46013}{24} \zeta_{9}-\frac{107245}{16} \zeta_{2} \zeta_{7}-\frac{17259}{4} \zeta_{3} \zeta_{6}-\frac{15539}{4} \zeta_{4} \zeta_{5}+\frac{143}{3}\left(\zeta_{3}\right)^{3}\right) h_{1}^{[1]} \\
& -\frac{354}{7} \zeta_{7,3}+\frac{1217}{5} \zeta_{2} \zeta_{5,3}-\frac{2668732849}{67200} \zeta_{10}-\frac{2659}{4} \zeta_{4}\left(\zeta_{3}\right)^{2}-3091 \zeta_{2} \zeta_{3} \zeta_{5} \\
& \left.+\frac{9179}{8} \zeta_{3} \zeta_{7}+\frac{20553}{28}\left(\zeta_{5}\right)^{2}\right\} .
\end{aligned}
$$

\section{NMHV results in the $3 \rightarrow 3$ self-crossing limit for $v>0$ through four loops}

In this appendix we give the results for the function $E$ entering the NMHV amplitude decomposition (2.14), in the $3 \rightarrow 3$ self-crossing limit for $v>0$. As predicted by the arguments in section 2.1, they are all nonsingular in this limit. We use the same notation as in the MHV case; the argument of the $h_{i}^{[w]}(z)$ is $z=1 / v$. Because $E(u, v, w)=E(w, v, u)$, there are two independent orientations of $E$ to quote at each loop order. We let $E$ refer to the limit $E(1+|\delta|, v, v)$, and $E^{\prime}$ to the limit $E(v, 1+|\delta|, v)$.

The results through three loops are

$$
\begin{aligned}
& E_{3 \rightarrow 3}^{(0)}(v>0)=1, \\
& E_{3 \rightarrow 3}^{\prime(0)}(v>0)=1, \\
& E_{3 \rightarrow 3}^{(1)}(v>0)=5 \zeta_{2}, \\
& E_{3 \rightarrow 3}^{\prime(1)}(v>0)=-2 h_{0}^{[2]}+5 \zeta_{2}-2 \pi i h_{0}^{[1]}, \\
& E_{3 \rightarrow 3}^{(2)}(v>0)=\frac{1}{2}\left[4 h_{8}^{[4]}+h_{10}^{[4]}-9 \zeta_{2} h_{2}^{[2]}+2 \zeta_{3} h_{1}^{[1]}-\frac{29}{4} \zeta_{4}+2 \pi i\left(2 h_{4}^{[3]}-3 \zeta_{3}\right)\right], \\
& E_{3 \rightarrow 3}^{\prime(2)}(v>0)=\frac{1}{2}\left[24 h_{0}^{[4]}+4 h_{2}^{[4]}+4 h_{4}^{[4]}+2 h_{6}^{[4]}+4 h_{8}^{[4]}+h_{10}^{[4]}\right. \\
& \quad-\zeta_{2}\left(56 h_{0}^{[2]}-2 h_{1}^{[2]}+9 h_{2}^{[2]}\right)+2 \zeta_{3}\left(h_{0}^{[1]}+h_{1}^{[1]}\right)-\frac{39}{4} \zeta_{4} \\
&\left.\quad+2 \pi i\left(12 h_{0}^{[3]}+2 h_{2}^{[3]}+2 h_{4}^{[3]}-4 \zeta_{2} h_{0}^{[1]}+\zeta_{3}\right)\right]
\end{aligned}
$$




$$
\begin{aligned}
E_{3 \rightarrow 3}^{(3)}(v>0)= & -24 h_{32}^{[6]}-5\left(h_{34}^{[6]}+h_{36}^{[6]}+h_{40}^{[6]}\right)-h_{42}^{[6]}+\frac{1}{2} \zeta_{2}\left(110 h_{8}^{[4]}+23 h_{10}^{[4]}\right) \\
& -\zeta_{3}\left(5 h_{4}^{[3]}+2 h_{5}^{[3]}\right)+15 \zeta_{4} h_{2}^{[2]}-\left(8 \zeta_{5}-23 \zeta_{2} \zeta_{3}\right) h_{1}^{[1]}+\frac{8729}{96} \zeta_{6}-\frac{3}{2}\left(\zeta_{3}\right)^{2} \\
& -\pi i\left[24 h_{16}^{[5]}+5\left(h_{18}^{[5]}+h_{20}^{[5]}\right)-7 \zeta_{2} h_{4}^{[3]}+5 \zeta_{3} h_{2}^{[2]}-25 \zeta_{5}+8 \zeta_{2} \zeta_{3}\right], \\
E_{3 \rightarrow 3}^{\prime(3)}(v>0)= & -120 h_{0}^{[6]}-24\left(h_{2}^{[6]}+h_{4}^{[6]}\right)-2 h_{6}^{[6]}-24 h_{8}^{[6]}-7 h_{10}^{[6]}-2 h_{12}^{[6]}-h_{14}^{[6]}-24 h_{16}^{[6]}-7 h_{18}^{[6]} \\
& -7 h_{20}^{[6]}-h_{22}^{[6]}-2 h_{24}^{[6]}-h_{26}^{[6]}-h_{28}^{[6]}-3 h_{30}^{[6]}-24 h_{32}^{[6]}-5 h_{34}^{[6]}-5 h_{36}^{[6]}-5 h_{40}^{[6]}-h_{42}^{[6]} \\
& +\zeta_{2}\left(276 h_{0}^{[4]}-2 h_{1}^{[4]}+53 h_{2}^{[4]}-h_{3}^{[4]}+53 h_{4}^{[4]}-h_{5}^{[4]}+4 h_{6}^{[4]}-3 h_{7}^{[4]}+55 h_{8}^{[4]}\right. \\
& \left.+\frac{23}{2} h_{10}^{[4]}\right)-\zeta_{3}\left(22 h_{0}^{[3]}+11 h_{1}^{[3]}+6 h_{2}^{[3]}-2 h_{3}^{[3]}+5 h_{4}^{[3]}+2 h_{5}^{[3]}\right) \\
+ & \frac{1}{4} \zeta_{4}\left(279 h_{0}^{[2]}+31 h_{1}^{[2]}+60 h_{2}^{[2]}\right)-2 \zeta_{5}\left(7 h_{0}^{[1]}+4 h_{1}^{[1]}\right)+\zeta_{2} \zeta_{3}\left(49 h_{0}^{[1]}+23 h_{1}^{[1]}\right) \\
+ & \frac{8477}{96} \zeta_{6}-3\left(\zeta_{3}\right)^{2}-\pi i\left[120 h_{0}^{[5]}+24\left(h_{2}^{[5]}+h_{4}^{[5]}\right)+2 h_{6}^{[5]}+24 h_{8}^{[5]}+7 h_{10}^{[5]}+2 h_{12}^{[5]}\right. \\
& +h_{14}^{[5]}+24 h_{16}^{[5]}+5 h_{18}^{[5]}+5 h_{20}^{[5]}-\zeta_{2}\left(36 h_{0}^{[3]}-2 h_{1}^{[3]}+5 h_{2}^{[3]}-h_{3}^{[3]}+7 h_{4}^{[3]}\right) \\
& \left.+\zeta_{3}\left(22 h_{0}^{[2]}+11 h_{1}^{[2]}+5 h_{2}^{[2]}\right)-\frac{39}{4} \zeta_{4} h_{0}^{[1]}+14 \zeta_{5}-5 \zeta_{2} \zeta_{3}\right] .
\end{aligned}
$$

The four-loop results are

$$
\begin{aligned}
E_{3 \rightarrow 3}^{(4)}(v>0)=\frac{1}{8}\{ & 2880 h_{128}^{[8]}+624\left(h_{130}^{[8]}+h_{132}^{[8]}\right)-48 h_{134}^{[8]}+624 h_{136}^{[8]}+152 h_{138}^{[8]}+28 h_{142}^{[8]} \\
& \left.+624 h_{144}^{[8]}+152 h_{146}^{[8]}+h_{148}^{[8]}\right)-4 h_{150}^{[8]}+8 h_{154}^{[8]}+16 h_{156}^{[8]}+6 h_{158}^{[8]} \\
& +624 h_{160}^{[8]}+152 h_{162}^{[8]}+152 h_{164}^{[8]}-4 h_{166}^{[8]}+152 h_{168}^{[8]}+42 h_{170}^{[8]}+8 h_{172}^{[8]} \\
& +3 h_{174}^{[8]}+8 h_{178}^{[8]}+8 h_{180}^{[8]}+16 h_{184}^{[8]}-18 h_{190}^{[8]} \\
& -\zeta_{2}\left(6576 h_{32}^{[6]}+48 h_{33}^{[6]}+1408 h_{34}^{[6]}-28 h_{35}^{[6]}+1408 h_{36}^{[6]}+4 h_{37}^{[6]}-8 h_{38}^{[6]}-6 h_{39}^{[6]}\right. \\
& \left.+1408 h_{40}^{[6]}+4 h_{41}^{[6]}+338 h_{42}^{[6]}-3 h_{43}^{[6]}-8 h_{44}^{[6]}+40 h_{46}^{[6]}+18 h_{47}^{[6]}\right) \\
+ & \zeta_{3}\left(672 h_{16}^{[5]}+276 h_{17}^{[5]}+156 h_{18}^{[5]}-6 h_{19}^{[5]}+156 h_{20}^{[5]}+73 h_{21}^{[5]}+8 h_{22}^{[5]}+18 h_{23}^{[5]}\right) \\
& -\zeta_{4}\left(1986 h_{8}^{[4]}-21 h_{9}^{[4]}+\frac{1053}{2} h_{10}^{[4]}+102 h_{11}^{[4]}\right)+\zeta_{5}\left(496 h_{4}^{[3]}+225 h_{5}^{[3]}\right) \\
& -\zeta_{2} \zeta_{3}\left(1524 h_{4}^{[3]}+627 h_{5}^{[3]}\right)-\left(\frac{16151}{8} \zeta_{6}-86\left(\zeta_{3}\right)^{2}\right) h_{2}^{[2]} \\
+ & \left(\frac{12135}{16} \zeta_{7}-\frac{4483}{2} \zeta_{2} \zeta_{5}-957 \zeta_{3} \zeta_{4}\right) h_{1}^{[1]} \\
& -\frac{9}{5} \zeta_{5,3}-\frac{12574711}{1440} \zeta_{8}+374 \zeta_{3} \zeta_{5}-566 \zeta_{2}\left(\zeta_{3}\right)^{2} \\
+ & 2 \pi i\left[1440 h_{64}^{[7]}+312\left(h_{66}^{[7]}+h_{68}^{[7]}+h_{72}^{[7]}\right)+76 h_{74}^{[7]}+8 h_{78}^{[7]}+312 h_{80}^{[7]}\right. \\
& \quad+76\left(h_{82}^{[7]}+h_{84}^{[7]}\right)+4\left(h_{86}^{[7]}+h_{90}^{[7]}\right)+8 h_{92}^{[7]} \\
& \quad-\zeta_{2}\left(408 h_{16}^{[5]}+80 h_{18}^{[5]}-8 h_{19}^{[5]}+80 h_{20}^{[5]}-4 h_{21}^{[5]}-4 h_{22}^{[5]}\right) \\
& +\zeta_{3}\left(336 h_{8}^{[4]}+144 h_{9}^{[4]}+78 h_{10}^{[4]}\right)-153 \zeta_{4} h_{4}^{[3]}+\left(248 \zeta_{5}-90 \zeta_{2} \zeta_{3}\right) h_{2}^{[2]} \\
& \left.\left.+12\left(\zeta_{3}\right)^{2} h_{1}^{[1]}-\frac{4041}{4} \zeta_{7}+330 \zeta_{2} \zeta_{5}+\frac{275}{2} \zeta_{3} \zeta_{4}\right]\right\} \\
& {[\mathrm{D} .9) } \\
& +9)
\end{aligned}
$$


and

$$
\begin{aligned}
& E_{3 \rightarrow 3}^{\prime(4)}(v>0)=\frac{1}{8}\left\{13440 h_{0}^{[8]}+2880\left(h_{2}^{[8]}+h_{4}^{[8]}\right)-160 h_{6}^{[8]}+2880 h_{8}^{[8]}+744 h_{10}^{[8]}+56 h_{12}^{[8]}\right. \\
& +140 h_{14}^{[8]}+2880 h_{16}^{[8]}+744\left(h_{18}^{[8]}+h_{20}^{[8]}\right)-12 h_{22}^{[8]}+80 h_{24}^{[8]}+60 h_{26}^{[8]}+80 h_{28}^{[8]} \\
& +60 h_{30}^{[8]}+2880 h_{32}^{[8]}+736\left(h_{34}^{[8]}+h_{36}^{[8]}\right)-16 h_{38}^{[8]}+736 h_{40}^{[8]}+196 h_{42}^{[8]}+32 h_{44}^{[8]} \\
& +56 h_{46}^{[8]}+96 h_{48}^{[8]}+56\left(h_{50}^{[8]}+h_{52}^{[8]}\right)+32 h_{54}^{[8]}+64 h_{56}^{[8]}+32\left(h_{58}^{[8]}+h_{60}^{[8]}\right) \\
& -12 h_{62}^{[8]}+2880 h_{64}^{[8]}+720\left(h_{66}^{[8]}+h_{68}^{[8]}\right)-16 h_{70}^{[8]}+720 h_{72}^{[8]}+192 h_{74}^{[8]}+32 h_{76}^{[8]} \\
& +60 h_{78}^{[8]}+720 h_{80}^{[8]}+192\left(h_{82}^{[8]}+h_{84}^{[8]}\right)+28 h_{86}^{[8]}+32 h_{88}^{[8]}+40 h_{90}^{[8]}+48 h_{92}^{[8]} \\
& +42 h_{94}^{[8]}+96 h_{96}^{[8]}+48\left(h_{98}^{[8]}+h_{100}^{[8]}\right)+32 h_{102}^{[8]}+48 h_{104}^{[8]}+36 h_{106}^{[8]} \\
& +32\left(h_{108}^{[8]}+h_{110}^{[8]}\right)+48 h_{112}^{[8]}+32\left(h_{114}^{[8]}+h_{116}^{[8]}+h_{118}^{[8]}+h_{120}^{[8]}+h_{122}^{[8]}\right) \\
& +24 h_{124}^{[8]}+120 h_{126}^{[8]}+2880 h_{128}^{[8]}+624\left(h_{130}^{[8]}+h_{132}^{[8]}\right)-48 h_{134}^{[8]}+624 h_{136}^{[8]} \\
& +152 h_{138}^{[8]}+28 h_{142}^{[8]}+624 h_{144}^{[8]}+152\left(h_{146}^{[8]}+h_{148}^{[8]}\right)-4 h_{150}^{[8]}+8 h_{154}^{[8]} \\
& +16 h_{156}^{[8]}+6 h_{158}^{[8]}+624 h_{160}^{[8]}+152\left(h_{162}^{[8]}+h_{164}^{[8]}\right)-4 h_{166}^{[8]}+152 h_{168}^{[8]} \\
& +42 h_{170}^{[8]}+8 h_{172}^{[8]}+3 h_{174}^{[8]}+8 h_{178}^{[8]}+8 h_{180}^{[8]}+16 h_{184}^{[8]}-18 h_{190}^{[8]} \\
& +\zeta_{2}\left(-30720 h_{0}^{[6]}-160 h_{1}^{[6]}-6456 h_{2}^{[6]}+140 h_{3}^{[6]}-6456 h_{4}^{[6]}-12 h_{5}^{[6]}-140 h_{6}^{[6]}+60 h_{7}^{[6]}\right. \\
& -6464 h_{8}^{[6]}-16 h_{9}^{[6]}-1644 h_{10}^{[6]}+56 h_{11}^{[6]}-184 h_{12}^{[6]}+32 h_{13}^{[6]}-128 h_{14}^{[6]}-12 h_{15}^{[6]} \\
& -6480 h_{16}^{[6]}-16 h_{17}^{[6]}-1608 h_{18}^{[6]}+60 h_{19}^{[6]}-1608 h_{20}^{[6]}+28 h_{21}^{[6]}-40 h_{22}^{[6]}+42 h_{23}^{[6]} \\
& -192 h_{24}^{[6]}+32 h_{25}^{[6]}-84 h_{26}^{[6]}+32 h_{27}^{[6]}-88 h_{28}^{[6]}+32 h_{29}^{[6]}-48 h_{30}^{[6]}+120 h_{31}^{[6]} \\
& -6576 h_{32}^{[6]}-48 h_{33}^{[6]}-1408 h_{34}^{[6]}+28 h_{35}^{[6]}-1408 h_{36}^{[6]}-4 h_{37}^{[6]}+8 h_{38}^{[6]}+6 h_{39}^{[6]} \\
& \left.-1408 h_{40}^{[6]}-4 h_{41}^{[6]}-338 h_{42}^{[6]}+3 h_{43}^{[6]}+8 h_{44}^{[6]}-40 h_{46}^{[6]}-18 h_{47}^{[6]}\right) \\
& +\zeta_{3}\left(3040 h_{0}^{[5]}+1292 h_{1}^{[5]}+756 h_{2}^{[5]}-20 h_{3}^{[5]}+752 h_{4}^{[5]}+304 h_{5}^{[5]}+24 h_{6}^{[5]}+44 h_{7}^{[5]}\right. \\
& +736 h_{8}^{[5]}+292 h_{9}^{[5]}+164 h_{10}^{[5]}-10 h_{11}^{[5]}+16 h_{12}^{[5]}+8 h_{13}^{[5]}-80 h_{15}^{[5]}+672 h_{16}^{[5]}+276 h_{17}^{[5]} \\
& \left.+156 h_{18}^{[5]}-6 h_{19}^{[5]}+156 h_{20}^{[5]}+73 h_{21}^{[5]}+8 h_{22}^{[5]}+18 h_{23}^{[5]}\right) \\
& -\zeta_{4}\left(9060 h_{0}^{[4]}+280 h_{1}^{[4]}+2545 h_{2}^{[4]}+356 h_{3}^{[4]}+2460 h_{4}^{[4]}+119 h_{5}^{[4]}+185 h_{6}^{[4]}+58 h_{7}^{[4]}\right. \\
& \left.+1986 h_{8}^{[4]}-21 h_{9}^{[4]}+\frac{1053}{2} h_{10}^{[4]}+102 h_{11}^{[4]}\right) \\
& +\zeta_{5}\left(2208 h_{0}^{[3]}+1068 h_{1}^{[3]}+548 h_{2}^{[3]}-64 h_{3}^{[3]}+496 h_{4}^{[3]}+225 h_{5}^{[3]}\right) \\
& -\zeta_{2} \zeta_{3}\left(6880 h_{0}^{[3]}+2920 h_{1}^{[3]}+1676 h_{2}^{[3]}+40 h_{3}^{[3]}+1524 h_{4}^{[3]}+627 h_{5}^{[3]}\right) \\
& -\zeta_{6}\left(\frac{28465}{3} h_{0}^{[2]}+\frac{1023}{4} h_{1}^{[2]}+\frac{16151}{8} h_{2}^{[2]}\right)+\left(\zeta_{3}\right)^{2}\left(392 h_{0}^{[2]}+39 h_{1}^{[2]}+86 h_{2}^{[2]}\right) \\
& +\frac{1}{16} \zeta_{7}\left(25120 h_{0}^{[1]}+12135 h_{1}^{[1]}\right)-\frac{1}{2} \zeta_{2} \zeta_{5}\left(9968 h_{0}^{[1]}+4483 h_{1}^{[1]}\right)-\zeta_{3} \zeta_{4}\left(2435 h_{0}^{[1]}+957 h_{1}^{[1]}\right) \\
& -\frac{2461055}{288} \zeta_{8}+578 \zeta_{3} \zeta_{5}-886 \zeta_{2}\left(\zeta_{3}\right)^{2} \\
& +2 \pi i\left[6720 h_{0}^{[7]}+1440 h_{2}^{[7]}+1440 h_{4}^{[7]}+28 h_{6}^{[7]}+1440 h_{8}^{[7]}+372 h_{10}^{[7]}+40 h_{12}^{[7]}+40 h_{14}^{[7]}\right. \\
& +1440 h_{16}^{[7]}+368 h_{18}^{[7]}+368 h_{20}^{[7]}+16 h_{22}^{[7]}+48 h_{24}^{[7]}+28 h_{26}^{[7]}+32 h_{28}^{[7]}+16 h_{30}^{[7]} \\
& +1440 h_{32}^{[7]}+360 h_{34}^{[7]}+360 h_{36}^{[7]}+16 h_{38}^{[7]}+360 h_{40}^{[7]}+96 h_{42}^{[7]}+16 h_{44}^{[7]}+24 h_{46}^{[7]} \\
& +48 h_{48}^{[7]}+24 h_{50}^{[7]}+24 h_{52}^{[7]}+16 h_{54}^{[7]}+24 h_{56}^{[7]}+16 h_{58}^{[7]}+16 h_{60}^{[7]}+12 h_{62}^{[7]} \\
& +1440 h_{64}^{[7]}+312 h_{66}^{[7]}+312 h_{68}^{[7]}+312 h_{72}^{[7]}+76 h_{74}^{[7]}+8 h_{78}^{[7]}+312 h_{80}^{[7]}+76 h_{82}^{[7]} \\
& +76 h_{84}^{[7]}+4 h_{86}^{[7]}+4 h_{90}^{[7]}+8 h_{92}^{[7]} \\
& +\zeta_{2}\left(-1920 h_{0}^{[5]}+28 h_{1}^{[5]}-348 h_{2}^{[5]}+40 h_{3}^{[5]}-352 h_{4}^{[5]}+16 h_{5}^{[5]}+4 h_{6}^{[5]}+16 h_{7}^{[5]}\right.
\end{aligned}
$$




$$
\begin{aligned}
& \quad-360 h_{8}^{[5]}+16 h_{9}^{[5]}-84 h_{10}^{[5]}+24 h_{11}^{[5]}+16 h_{13}^{[5]}+4 h_{14}^{[5]}+12 h_{15}^{[5]}-408 h_{16}^{[5]}-80 h_{18}^{[5]} \\
& \left.\quad+8 h_{19}^{[5]}-80 h_{20}^{[5]}+4 h_{21}^{[5]}+4 h_{22}^{[5]}\right) \\
& +\zeta_{3}\left(1520 h_{0}^{[4]}+664 h_{1}^{[4]}+376 h_{2}^{[4]}+8 h_{3}^{[4]}+368 h_{4}^{[4]}+152 h_{5}^{[4]}+8 h_{6}^{[4]}+4 h_{7}^{[4]}+336 h_{8}^{[4]}\right. \\
& \left.\quad+144 h_{9}^{[4]}+78 h_{10}^{[4]}\right) \\
& -\zeta_{4}\left(690 h_{0}^{[3]}-13 h_{1}^{[3]}+150 h_{2}^{[3]}-2 h_{3}^{[3]}+153 h_{4}^{[3]}\right)+\zeta_{5}\left(1104 h_{0}^{[2]}+516 h_{1}^{[2]}+248 h_{2}^{[2]}\right) \\
& -\zeta_{2} \zeta_{3}\left(400 h_{0}^{[2]}+172 h_{1}^{[2]}+90 h_{2}^{[2]}\right)-\frac{2635}{6} \zeta_{6} h_{0}^{[1]}+\left(\zeta_{3}\right)^{2}\left(196 h_{0}^{[1]}+12 h_{1}^{[1]}\right) \\
& \left.\left.+785 \zeta_{7}-284 \zeta_{2} \zeta_{5}-\frac{355}{2} \zeta_{3} \zeta_{4}\right]\right\} .
\end{aligned}
$$

The results for $E^{\prime}=E(v, 1+|\delta|, v)$ are more complicated than those for $E=E(1+$ $|\delta|, v, v)$, because only the latter orientation obeys a final entry condition that is compatible with the self-crossing line. Both sets of functions are smooth as $v \rightarrow 1^{+}$. While $E(1+$ $|\delta|, v, v)$ is also smooth as $v \rightarrow \infty, E(v, 1+|\delta|, v)$ has logarithmic divergences there. These are precisely the logarithms associated with the NMHV multi-particle factorization pole discussed in refs. [61, 62], where the function $U(u, v, w)=\ln E(u, v, w)$ was studied in the limit $u, w \rightarrow \infty$ with $v$ fixed.

In the remainder of this appendix we discuss the behavior of the full NMHV amplitude in the overlap region between the $2 \rightarrow 4$ multi-Regge limit and the self-crossing limit, $w \rightarrow-1$, where we can work to higher order in the expansion around the self-crossing limit. This is necessary because the rational prefactors, or $R$-invariants, "( $i)$ " in eq. (2.14), blow up in the self-crossing limit. The denominators of some of the $R$-invariants contain spinor strings such as $\left\langle 3^{-}|(1+2)| 6^{-}\right\rangle=\langle 3|(1+2)| 6]$. The square of this factor is

$$
\begin{aligned}
\left\langle 3^{-}|(1+2)| 6^{-}\right\rangle\left\langle 6^{-}|(1+2)| 3^{-}\right\rangle & =\frac{1}{2} \operatorname{tr}\left[\left(1-\gamma_{5}\right) 3(1+2) 6(1+2)\right] \\
& =s_{123} s_{345}-s_{12} s_{45}=(1-u) s_{123} s_{345} \\
& =\delta s_{123} s_{345} .
\end{aligned}
$$

This behavior leads some of the $R$-invariants to blow up like $1 /\langle 3|(1+2)| 6] \propto 1 / \sqrt{\delta}$.

On the other hand, in the Euclidean region this "spurious pole" power-law singularity is completely cancelled by a relation between the transcendental functions $E$ and $\tilde{E}$ [60-62]. Here we will see that in Minkowski kinematics a logarithmic singularity survives. Actually we will not do so for generic self-crossing kinematics, but only for $v \rightarrow 0$, making use of the overlap with the $w \rightarrow-1$ multi-Regge limit. For the generic multi-Regge limit, for the helicity configuration

$$
3^{+} 6^{+} \rightarrow 2^{+} 4^{-} 5^{+} 1^{+},
$$

an analysis of the behavior of the $R$-invariants [61] leads to the following formula for the BDS-like normalized amplitude:

$$
\begin{aligned}
\rho \equiv \frac{\mathcal{A}_{6}^{\mathrm{NMHV}}}{\mathcal{A}_{6}^{\mathrm{BDS}-\text { like }}=} & \frac{1}{1+w}[E(u, v, w)+\tilde{E}(u, v, w)+E(w, u, v)-\tilde{E}(w, u, v)] \\
& +\frac{w}{1+w}[E(v, w, u)+\tilde{E}(v, w, u)+E(w, u, v)-\tilde{E}(w, u, v)] .
\end{aligned}
$$


The $2 \rightarrow 4$ MRK behavior of the ratio function was provided through four loops in refs. [61, $62]$ in terms of SVHPLs [77]. Converting to the BDS-like normalized functions $E$ and $\tilde{E}$, and taking the limit $w \rightarrow-1$, we find through four loops:

$$
\begin{aligned}
& \rho^{(1)}=-\ln ^{2} v-2 \zeta_{2}+2 \pi i\left[-\ln v-1-\frac{1+w^{*}}{1+w}\right]+(2 \pi i)^{2}\left[-\frac{1}{2}\right], \\
& \rho^{(2)}=\frac{1}{4} \ln ^{4} v+2 \zeta_{2} \ln ^{2} v+\frac{15}{2} \zeta_{4} \\
& +(2 \pi i)\left[\frac{1}{2} \ln ^{2} \delta-\ln \delta+\frac{1}{2} \ln ^{3} v-4 \zeta_{2} \ln v+\zeta_{3}-4 \zeta_{2}+1\right. \\
& \left.+\frac{1+w^{*}}{1+w}\left(\frac{1}{2} \ln ^{2} v+\ln v-4 \zeta_{2}+1\right)\right] \\
& +(2 \pi i)^{2}\left[\frac{1}{2} \ln \delta+\frac{1}{2} \ln ^{2} v-\frac{1}{2} \zeta_{2}-\frac{1}{2}+\frac{1+w^{*}}{1+w} \frac{1}{2}(\ln v+1)\right], \\
& \rho^{(3)}=-\frac{1}{24} \ln ^{6} v-\frac{3}{4} \zeta_{2} \ln ^{4} v-\frac{47}{4} \zeta_{4} \ln ^{2} v-\frac{707}{24} \zeta_{6} \\
& +(2 \pi i)\left[-\frac{1}{8} \ln ^{4} \delta+\frac{1}{2} \ln ^{3} \delta+\left(\frac{9}{2} \zeta_{2}-\frac{3}{2}\right) \ln ^{2} \delta+\left(\zeta_{3}-9 \zeta_{2}+3\right) \ln \delta\right. \\
& -\frac{1}{8} \ln ^{5} v+\frac{5}{2} \zeta_{2} \ln ^{3} v+\frac{1}{2} \zeta_{3} \ln ^{2} v-\frac{47}{4} \zeta_{4} \ln v-7 \zeta_{5}-\frac{47}{4} \zeta_{4}-\zeta_{3}+9 \zeta_{2}-3 \\
& +\frac{1+w^{*}}{1+w}\left(-\frac{1}{8} \ln ^{4} v-\frac{1}{2} \ln ^{3} v+\left(\frac{9}{2} \zeta_{2}-\frac{3}{2}\right) \ln ^{2} v-\left(\zeta_{3}-9 \zeta_{2}+3\right) \ln v\right. \\
& \left.\left.-\frac{47}{4} \zeta_{4}-\zeta_{3}+9 \zeta_{2}-3\right)\right] \\
& +(2 \pi i)^{2}\left[-\frac{1}{4} \ln ^{3} \delta+\frac{3}{4} \ln ^{2} \delta+\frac{3}{2}\left(\zeta_{2}-1\right) \ln \delta-\frac{3}{16} \ln ^{4} v+\frac{3}{4} \zeta_{2} \ln ^{2} v+\frac{1}{2} \zeta_{3} \ln v\right. \\
& -\frac{17}{8} \zeta_{4}+\frac{1}{2} \zeta_{3}-\frac{3}{2} \zeta_{2}+\frac{3}{2} \\
& \left.+\frac{1+w^{*}}{1+w}\left(-\frac{1}{4} \ln ^{3} v-\frac{3}{4} \ln ^{2} v+\frac{3}{2}\left(\zeta_{2}-1\right) \ln v-\frac{1}{2} \zeta_{3}+\frac{3}{2} \zeta_{2}-\frac{3}{2}\right)\right], \\
& \rho^{(4)}=\frac{1}{192} \ln ^{8} v+\frac{1}{6} \zeta_{2} \ln ^{6} v+\frac{89}{16} \zeta_{4} \ln ^{4} v+\left(\frac{1399}{24} \zeta_{6}+\left(\zeta_{3}\right)^{2}\right) \ln ^{2} v+\frac{39895}{288} \zeta_{8}+2 \zeta_{2}\left(\zeta_{3}\right)^{2} \\
& +(2 \pi i)\left[\frac{1}{48} \ln ^{6} \delta-\frac{1}{8} \ln ^{5} \delta+\left(-\frac{7}{4} \zeta_{2}+\frac{5}{8}\right) \ln ^{4} \delta+\left(\frac{1}{6} \zeta_{3}+7 \zeta_{2}-\frac{5}{2}\right) \ln ^{3} \delta\right. \\
& +\left(\frac{149}{8} \zeta_{4}-\frac{1}{2} \zeta_{3}-21 \zeta_{2}+\frac{15}{2}\right) \ln ^{2} \delta+\left(-4 \zeta_{5}-\frac{149}{4} \zeta_{4}+\zeta_{3}+42 \zeta_{2}-15\right) \ln \delta \\
& +\frac{1}{48} \ln ^{7} v-\frac{3}{4} \zeta_{2} \ln ^{5} v-\frac{3}{8} \zeta_{3} \ln ^{4} v+\frac{69}{8} \zeta_{4} \ln ^{3} v+\left(-\frac{25}{8} \zeta_{5}+\frac{23}{2} \zeta_{2} \zeta_{3}\right) \ln ^{2} v \\
& +\left(-\frac{107}{6} \zeta_{6}+\frac{5}{2}\left(\zeta_{3}\right)^{2}\right) \ln v+\frac{1381}{32} \zeta_{7}-\frac{31}{4} \zeta_{3} \zeta_{4}-\frac{11}{4} \zeta_{2} \zeta_{5}-\frac{107}{6} \zeta_{6}+2\left(\zeta_{3}\right)^{2} \\
& +4 \zeta_{5}+\frac{149}{4} \zeta_{4}-\zeta_{3}-42 \zeta_{2}+15 \\
& +\frac{1+w^{*}}{1+w}\left(\frac{1}{48} \ln ^{6} v+\frac{1}{8} \ln ^{5} v+\left(-\frac{7}{4} \zeta_{2}+\frac{5}{8}\right) \ln ^{4} v+\left(-\frac{1}{12} \zeta_{3}-7 \zeta_{2}+\frac{5}{2}\right) \ln ^{3} v\right. \\
& +\left(\frac{149}{8} \zeta_{4}-\frac{1}{4} \zeta_{3}-21 \zeta_{2}+\frac{15}{2}\right) \ln ^{2} v \\
& +\left(5 \zeta_{5}+\frac{1}{2} \zeta_{2} \zeta_{3}+\frac{149}{4} \zeta_{4}-\frac{1}{2} \zeta_{3}-42 \zeta_{2}+15\right) \ln v-\frac{107}{6} \zeta_{6}+\frac{5}{2}\left(\zeta_{3}\right)^{2}
\end{aligned}
$$




$$
\begin{gathered}
\left.\left.+5 \zeta_{5}+\frac{1}{2} \zeta_{2} \zeta_{3}+\frac{149}{4} \zeta_{4}-\frac{1}{2} \zeta_{3}-42 \zeta_{2}+15\right)\right] \\
+(2 \pi i)^{2}\left[\frac{1}{16} \ln ^{5} \delta-\frac{5}{16} \ln ^{4} \delta+\left(-\zeta_{2}+\frac{5}{4}\right) \ln ^{3} \delta+\left(\frac{1}{4} \zeta_{3}+3 \zeta_{2}-\frac{15}{4}\right) \ln ^{2} \delta\right. \\
+\left(\frac{29}{8} \zeta_{4}-\frac{1}{2} \zeta_{3}-6 \zeta_{2}+\frac{15}{2}\right) \ln \delta+\frac{1}{24} \ln ^{6} v-\frac{5}{16} \zeta_{2} \ln ^{4} v-\frac{3}{4} \zeta_{3} \ln ^{3} v \\
+\frac{29}{8} \zeta_{4} \ln ^{2} v+\left(-\frac{25}{8} \zeta_{5}+\frac{5}{2} \zeta_{2} \zeta_{3}\right) \ln v+\frac{341}{96} \zeta_{6}+\frac{5}{4}\left(\zeta_{3}\right)^{2}-2 \zeta_{5}+\zeta_{2} \zeta_{3} \\
-\frac{29}{8} \zeta_{4}+\frac{1}{2} \zeta_{3}+6 \zeta_{2}-\frac{15}{2} \\
+\frac{1+w^{*}}{1+w}\left(\frac{1}{16} \ln ^{5} v+\frac{5}{16} \ln ^{4} v+\left(-\zeta_{2}+\frac{5}{4}\right) \ln ^{3} v+\left(-\frac{1}{8} \zeta_{3}-3 \zeta_{2}+\frac{15}{4}\right) \ln ^{2} v\right. \\
+\left(\frac{29}{8} \zeta_{4}-\frac{1}{4} \zeta_{3}-6 \zeta_{2}+\frac{15}{2}\right) \ln v+\frac{5}{2} \zeta_{5}-\frac{1}{4} \zeta_{2} \zeta_{3} \\
\left.\left.+\frac{29}{8} \zeta_{4}-\frac{1}{4} \zeta_{3}-6 \zeta_{2}+\frac{15}{2}\right)\right] .
\end{gathered}
$$

In these expressions we let $(1+w) \rightarrow \xi(1+w),\left(1+w^{*}\right) \rightarrow \xi\left(1+w^{*}\right)$, take $\xi \rightarrow 0$, and drop terms that vanish in this limit. The only two rational prefactors that can survive are then 1 and $\left(1+w^{*}\right) /(1+w)$. The results do not have uniform transcendentality because the transcendental functions have been expanded to higher order around the $w \rightarrow-1$ limit to cancel the $1 /(1+w)$ factor in eq. (D.13). We rewrite the logarithmic terms using the self-crossing variables, i.e. we let $\ln |1+w|^{2} \rightarrow \ln \delta-\ln v$. We see that there are indeed logarithmically singular $\ln \delta$ terms in the imaginary part and in the double discontinuity $(2 \pi i)^{2}$ term, beginning at two loops. However, there are no $\ln \delta$ terms in the part with the $\left(1+w^{*}\right) /(1+w)$ prefactor; that is, the terms depending on the azimuthal component of the vector $\vec{z}$ in eq. (2.22) are finite. Furthermore, the $\ln \delta$ terms contain no $v$ dependence (which could only appear through powers of $\ln v$ in the approximation in which we are working). This behavior is reminiscent of what we found for the MHV configuration. It would be very interesting to try to understand these NMHV properties better, both in the MRK limit and more generally along the full self-crossing line.

\section{E Seven-point self-crossing kinematics}

In this section we briefly describe the self-crossing configuration for the seven-point amplitude, or heptagonal Wilson loop shown in figure 12. We consider

$$
\begin{aligned}
k_{3}+k_{7} & \rightarrow k_{1}+k_{2}+k_{4}+k_{5}+k_{6}, \\
(1-x) k_{3}+(1-y) k_{7} & \rightarrow k_{1}+k_{2}, \\
x k_{3}+y k_{7} & \rightarrow k_{4}+k_{5}+k_{6} .
\end{aligned}
$$

Incoming gluons 3 and 7 split into collinear pairs with momentum fractions $x$ and $1-x$, and $y$ and $1-y$, respectively. These pairs then undergo a $2 \rightarrow 2$ scattering into final state gluons 1,2 , and a $2 \rightarrow 3$ scattering into final state gluons 4,5 and 6 . 


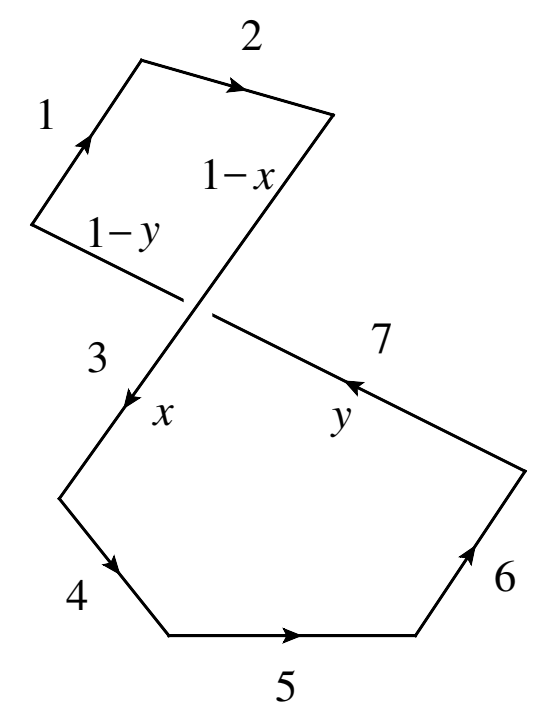

Figure 12. The self-crossing configuration for $2 \rightarrow 5$ scattering, where particles 3 and 7 are incoming, and 1, 2, 4, 5 and 6 are outgoing.

It is straightforward to derive the following relations among the Mandelstam variables:

$$
\begin{aligned}
s_{12} & =(1-x)(1-y) s_{37}, \\
(1-x) s_{23} & =(1-y) s_{71}, \\
s_{456} & =x y s_{37}, \\
s_{123} & =-x(1-y) s_{37}, \\
s_{712} & =-(1-x) y s_{37}, \\
x s_{34} & =(1-y) s_{56}+y s_{567}, \\
y s_{67} & =(1-x) s_{45}+x s_{345} .
\end{aligned}
$$

We would like to rewrite these constraints in terms of the seven dual conformal cross ratios defined in ref. [85],

or

$$
u_{i} \equiv u_{i, i+3}=\frac{x_{i, i+4}^{2} x_{i+1, i+3}^{2}}{x_{i, i+3}^{2} x_{i+1, i+4}^{2}}
$$

$$
u_{1}=\frac{x_{15}^{2} x_{24}^{2}}{x_{14}^{2} x_{25}^{2}}=\frac{s_{23} s_{567}}{s_{123} s_{234}}
$$

and cyclic permutations thereof $(\bmod 7)$.

It is easy to see that

$$
u_{7}=\frac{s_{12} s_{456}}{s_{712} s_{123}}=1,
$$

which is the analog of the $u=1$ constraint in the six-point case.

To find the second constraint on the cross ratios, analogous to $v=w$ in the six-point case, we first look for combinations of cross ratios from which the invariants $s_{234}$ and $s_{671}$ are absent:

$$
\frac{u_{1}}{u_{2} u_{6}}=\frac{s_{23}}{s_{71}} \frac{s_{712}}{s_{123}} \frac{s_{567}}{s_{34}}=\frac{y}{x} \frac{s_{567}}{s_{34}},
$$




$$
\begin{aligned}
\frac{u_{6}}{u_{1} u_{5}} & =\frac{s_{71}}{s_{23}} \frac{s_{123}}{s_{712}} \frac{s_{345}}{s_{67}}=\frac{x}{y} \frac{s_{345}}{s_{67}}, \\
\frac{u_{3} u_{4}}{u_{2} u_{5}} & =\frac{s_{45} s_{56} s_{712} s_{123}}{s_{34} s_{67} s_{456}^{2}}=\frac{(1-y) s_{56}}{x s_{34}} \cdot \frac{(1-x) s_{45}}{y s_{67}} .
\end{aligned}
$$

Using eqs. (E.9) and (E.10), we can rewrite eq. (E.16) as

$$
\frac{u_{3} u_{4}}{u_{2} u_{5}}=\left[1-\frac{y s_{567}}{x s_{34}}\right]\left[1-\frac{x s_{345}}{y s_{67}}\right]=\left[1-\frac{u_{1}}{u_{2} u_{6}}\right]\left[1-\frac{u_{6}}{u_{1} u_{5}}\right],
$$

or

$$
\left(u_{1} u_{5}-u_{6}\right)\left(u_{2} u_{6}-u_{1}\right)=u_{1} u_{3} u_{4} u_{6} .
$$

We solve eq. (E.18) for $u_{4}$, and insert that solution and $u_{7}=1$ into the Gram determinant constraint that is obeyed by the seven cross ratios to have four-dimensional kinematics. We find that the Gram determinant vanishing condition then contains a simple factor, $u_{6}-u_{1} u_{5}-u_{3} u_{6}$. Setting this factor to zero, we solve for $u_{3}$ and plug the solution back into eq. (E.18). We obtain

$$
u_{7}=1, \quad u_{3}=1-\frac{u_{1} u_{5}}{u_{6}}, \quad u_{4}=1-\frac{u_{2} u_{6}}{u_{1}} .
$$

The self-crossing solution is then parametrized by the four remaining cross ratios, $u_{1}, u_{2}$, $u_{5}$ and $u_{6}$.

Open Access. This article is distributed under the terms of the Creative Commons Attribution License (CC-BY 4.0), which permits any use, distribution and reproduction in any medium, provided the original author(s) and source are credited.

\section{References}

[1] L.D. Landau, On analytic properties of vertex parts in quantum field theory, Nucl. Phys. 13 (1959) 181 [InSPIRE].

[2] J.D. Bjorken and S.D. Drell, Relativistic quantum field theory, McGraw-Hill, New York U.S.A. (1965).

[3] S. Coleman and R.E. Norton, Singularities in the physical region, Nuovo Cim. 38 (1965) 438 [INSPIRE].

[4] Z. Nagy and D.E. Soper, Numerical integration of one-loop Feynman diagrams for N-photon amplitudes, Phys. Rev. D 74 (2006) 093006 [hep-ph/0610028] [INSPIRE].

[5] R.K. Ellis and G. Zanderighi, Scalar one-loop integrals for QCD, JHEP 02 (2008) 002 [arXiv: 0712.1851] [INSPIRE].

[6] L.D. Ninh, One-loop Yukawa corrections to the process $p p \rightarrow b \bar{b} H$ in the Standard Model at the LHC: Landau singularities, arXiv:0810.4078 [INSPIRE].

[7] J.R. Gaunt and W.J. Stirling, Double parton scattering singularity in one-loop integrals, JHEP 06 (2011) 048 [arXiv: 1103.1888] [INSPIRE].

[8] M. Diehl, D. Ostermeier and A. Schafer, Elements of a theory for multiparton interactions in QCD, JHEP 03 (2012) 089 [arXiv:1111.0910] [INSPIRE]. 
[9] T. Dennen, M. Spradlin and A. Volovich, Landau singularities and symbology: one- and two-loop MHV amplitudes in SYM theory, JHEP 03 (2016) 069 [arXiv: 1512.07909] [INSPIRE].

[10] M. Cacciari, G.P. Salam and S. Sapeta, On the characterisation of the underlying event, JHEP 04 (2010) 065 [arXiv:0912.4926] [InSPIRE].

[11] B. Blok, Yu. Dokshitzer, L. Frankfurt and M. Strikman, The four jet production at LHC and Tevatron in QCD, Phys. Rev. D 83 (2011) 071501 [arXiv:1009.2714] [INSPIRE].

[12] M.G. Ryskin and A.M. Snigirev, A fresh look at double parton scattering, Phys. Rev. D 83 (2011) 114047 [arXiv:1103.3495] [INSPIRE].

[13] B. Blok, Yu. Dokshitser, L. Frankfurt and M. Strikman, $p Q C D$ physics of multiparton interactions, Eur. Phys. J. C 72 (2012) 1963 [arXiv:1106.5533] [INSPIRE].

[14] M.G. Ryskin and A.M. Snigirev, Double parton scattering in double logarithm approximation of perturbative QCD, Phys. Rev. D 86 (2012) 014018 [arXiv:1203.2330] [INSPIRE].

[15] B. Blok, Yu. Dokshitzer, L. Frankfurt and M. Strikman, Perturbative QCD correlations in multi-parton collisions, Eur. Phys. J. C 74 (2014) 2926 [arXiv:1306.3763] [INSPIRE].

[16] M. Diehl, Multiparton interactions: theory and experimental findings, PoS(DIS 2013) 006 [arXiv:1306.6059] [INSPIRE].

[17] L. Brink, J.H. Schwarz and J. Scherk, Supersymmetric Yang-Mills theories, Nucl. Phys. B 121 (1977) 77 [inSPIRE].

[18] N. Beisert et al., Review of AdS/CFT integrability: an overview, Lett. Math. Phys. 99 (2012) 3 [arXiv:1012.3982] [INSPIRE].

[19] J.M. Drummond, J. Henn, V.A. Smirnov and E. Sokatchev, Magic identities for conformal four-point integrals, JHEP 01 (2007) 064 [hep-th/0607160] [INSPIRE].

[20] Z. Bern, M. Czakon, L.J. Dixon, D.A. Kosower and V.A. Smirnov, The four-loop planar amplitude and cusp anomalous dimension in maximally supersymmetric Yang-Mills theory, Phys. Rev. D 75 (2007) 085010 [hep-th/0610248] [INSPIRE].

[21] Z. Bern, J.J.M. Carrasco, H. Johansson and D.A. Kosower, Maximally supersymmetric planar Yang-Mills amplitudes at five loops, Phys. Rev. D 76 (2007) 125020 [arXiv: 0705.1864] [INSPIRE].

[22] L.F. Alday and J.M. Maldacena, Gluon scattering amplitudes at strong coupling, JHEP 06 (2007) 064 [arXiv:0705.0303] [INSPIRE].

[23] J.M. Drummond, J. Henn, G.P. Korchemsky and E. Sokatchev, Dual superconformal symmetry of scattering amplitudes in $N=4$ super-Yang-Mills theory, Nucl. Phys. B 828 (2010) 317 [arXiv:0807.1095] [inSPIRE].

[24] J.M. Drummond, G.P. Korchemsky and E. Sokatchev, Conformal properties of four-gluon planar amplitudes and Wilson loops, Nucl. Phys. B 795 (2008) 385 [arXiv:0707.0243] [INSPIRE].

[25] A. Brandhuber, P. Heslop and G. Travaglini, $M H V$ amplitudes in $N=4$ super Yang-Mills and Wilson loops, Nucl. Phys. B 794 (2008) 231 [arXiv:0707.1153] [INSPIRE].

[26] L.F. Alday and R. Roiban, Scattering amplitudes, Wilson loops and the string/gauge theory correspondence, Phys. Rept. 468 (2008) 153 [arXiv:0807.1889] [InSPIRE].

[27] T. Adamo, M. Bullimore, L. Mason and D. Skinner, Scattering amplitudes and Wilson loops in twistor space, J. Phys. A 44 (2011) 454008 [arXiv:1104.2890] [InSPIRE]. 
[28] G. Georgiou, Null Wilson loops with a self-crossing and the Wilson loop/amplitude conjecture, JHEP 09 (2009) 021 [arXiv:0904.4675] [INSPIRE].

[29] H. Dorn and S. Wuttke, Wilson loop remainder function for null polygons in the limit of self-crossing, JHEP 05 (2011) 114 [arXiv:1104.2469] [INSPIRE].

[30] H. Dorn and S. Wuttke, Hexagon remainder function in the limit of self-crossing up to three loops, JHEP 04 (2012) 023 [arXiv:1111.6815] [INSPIRE].

[31] G.P. Korchemsky, On near forward high-energy scattering in QCD, Phys. Lett. B 325 (1994) 459 [hep-ph/9311294] [INSPIRE].

[32] I.A. Korchemskaya and G.P. Korchemsky, High-energy scattering in $Q C D$ and cross singularities of Wilson loops, Nucl. Phys. B 437 (1995) 127 [hep-ph/9409446] [INSPIRE].

[33] R.A. Brandt, F. Neri and M.-A. Sato, Renormalization of loop functions for all loops, Phys. Rev. D 24 (1981) 879 [INSPIRE].

[34] R.A. Brandt, A. Gocksch, M.-A. Sato and F. Neri, Loop space, Phys. Rev. D 26 (1982) 3611 [INSPIRE].

[35] J. Bartels, L.N. Lipatov and A. Sabio Vera, BFKL pomeron, reggeized gluons and Bern-Dixon-Smirnov amplitudes, Phys. Rev. D 80 (2009) 045002 [arXiv:0802.2065] [INSPIRE].

[36] J. Bartels, L.N. Lipatov and A. Sabio Vera, $N=4$ supersymmetric Yang-Mills scattering amplitudes at high energies: the Regge cut contribution, Eur. Phys. J. C 65 (2010) 587 [arXiv: 0807.0894] [INSPIRE].

[37] L.N. Lipatov and A. Prygarin, Mandelstam cuts and light-like Wilson loops in $N=4$ SUSY, Phys. Rev. D 83 (2011) 045020 [arXiv: 1008.1016] [INSPIRE].

[38] L.N. Lipatov and A. Prygarin, BFKL approach and six-particle $M H V$ amplitude in $N=4$ super Yang-Mills, Phys. Rev. D 83 (2011) 125001 [arXiv:1011.2673] [INSPIRE].

[39] J. Bartels, L.N. Lipatov and A. Prygarin, $M H V$ amplitude for $3 \rightarrow 3$ gluon scattering in Regge limit, Phys. Lett. B 705 (2011) 507 [arXiv: 1012.3178] [INSPIRE].

[40] J. Bartels, L.N. Lipatov and A. Prygarin, Collinear and Regge behavior of $2 \rightarrow 4 M H V$ amplitude in $N=4$ super Yang-Mills theory, arXiv:1104.4709 [INSPIRE].

[41] L.J. Dixon, J.M. Drummond and J.M. Henn, Bootstrapping the three-loop hexagon, JHEP 11 (2011) 023 [arXiv:1108.4461] [INSPIRE].

[42] V.S. Fadin and L.N. Lipatov, BFKL equation for the adjoint representation of the gauge group in the next-to-leading approximation at $N=4$ SUSY, Phys. Lett. B 706 (2012) 470 [arXiv:1111.0782] [INSPIRE].

[43] L. Lipatov, A. Prygarin and H.J. Schnitzer, The multi-Regge limit of NMHV amplitudes in $N=4$ SYM theory, JHEP 01 (2013) 068 [arXiv:1205.0186] [INSPIRE].

[44] L.J. Dixon, C. Duhr and J. Pennington, Single-valued harmonic polylogarithms and the multi-Regge limit, JHEP 10 (2012) 074 [arXiv:1207.0186] [INSPIRE].

[45] J. Pennington, The six-point remainder function to all loop orders in the multi-Regge limit, JHEP 01 (2013) 059 [arXiv:1209.5357] [INSPIRE].

[46] S. Caron-Huot, When does the gluon reggeize?, JHEP 05 (2015) 093 [arXiv:1309.6521] [INSPIRE]. 
[47] L.J. Dixon, J.M. Drummond, C. Duhr and J. Pennington, The four-loop remainder function and multi-Regge behavior at NNLLA in planar $N=4$ super-Yang-Mills theory, JHEP 06 (2014) 116 [arXiv:1402.3300] [INSPIRE].

[48] Y. Hatsuda, Wilson loop OPE, analytic continuation and multi-Regge limit, JHEP 10 (2014) 038 [arXiv: 1404.6506] [INSPIRE].

[49] B. Basso, S. Caron-Huot and A. Sever, Adjoint BFKL at finite coupling: a short-cut from the collinear limit, JHEP 01 (2015) 027 [arXiv:1407.3766] [INSPIRE].

[50] J.M. Drummond and G. Papathanasiou, Hexagon OPE resummation and multi-Regge kinematics, JHEP 02 (2016) 185 [arXiv:1507.08982] [INSPIRE].

[51] J. Broedel and M. Sprenger, Six-point remainder function in multi-Regge-kinematics: an efficient approach in momentum space, JHEP 05 (2016) 055 [arXiv: 1512.04963] [INSPIRE].

[52] T. Bargheer, G. Papathanasiou and V. Schomerus, The two-loop symbol of all multi-Regge regions, JHEP 05 (2016) 012 [arXiv: 1512.07620] [INSPIRE].

[53] B. Basso, A. Sever and P. Vieira, Spacetime and flux tube S-matrices at finite coupling for $N=4$ supersymmetric Yang-Mills theory, Phys. Rev. Lett. 111 (2013) 091602 [arXiv:1303.1396] [INSPIRE].

[54] B. Basso, A. Sever and P. Vieira, Space-time S-matrix and flux tube S-matrix II. Extracting and matching data, JHEP 01 (2014) 008 [arXiv:1306.2058] [INSPIRE].

[55] B. Basso, A. Sever and P. Vieira, Space-time S-matrix and flux-tube S-matrix III. The two-particle contributions, JHEP 08 (2014) 085 [arXiv: 1402.3307] [INSPIRE].

[56] B. Basso, A. Sever and P. Vieira, Space-time S-matrix and flux-tube S-matrix IV. Gluons and fusion, JHEP 09 (2014) 149 [arXiv:1407.1736] [INSPIRE].

[57] S. Caron-Huot, L.J. Dixon, M. von Hippel and A.J. McLeod, Bootstrapping a five-loop amplitude from Steinmann relations, to appear.

[58] A.B. Goncharov, M. Spradlin, C. Vergu and A. Volovich, Classical polylogarithms for amplitudes and Wilson loops, Phys. Rev. Lett. 105 (2010) 151605 [arXiv:1006.5703] [INSPIRE].

[59] L.J. Dixon, J.M. Drummond, M. von Hippel and J. Pennington, Hexagon functions and the three-loop remainder function, JHEP 12 (2013) 049 [arXiv: 1308.2276] [INSPIRE].

[60] L.J. Dixon, J.M. Drummond and J.M. Henn, Analytic result for the two-loop six-point NMHV amplitude in $N=4$ super Yang-Mills theory, JHEP 01 (2012) 024 [arXiv:1111.1704] [INSPIRE].

[61] L.J. Dixon and M. von Hippel, Bootstrapping an NMHV amplitude through three loops, JHEP 10 (2014) 065 [arXiv: 1408.1505] [INSPIRE].

[62] L.J. Dixon, M. von Hippel and A.J. McLeod, The four-loop six-gluon NMHV ratio function, JHEP 01 (2016) 053 [arXiv:1509.08127] [INSPIRE].

[63] Z. Bern, L.J. Dixon and V.A. Smirnov, Iteration of planar amplitudes in maximally supersymmetric Yang-Mills theory at three loops and beyond, Phys. Rev. D 72 (2005) 085001 [hep-th/0505205] [INSPIRE].

[64] S.J. Parke and T.R. Taylor, An amplitude for $n$ gluon scattering, Phys. Rev. Lett. 56 (1986) 2459 [INSPIRE].

[65] M.L. Mangano, S.J. Parke and Z. Xu, Duality and multi-gluon scattering, Nucl. Phys. B 298 (1988) 653 [inSPIRE]. 
[66] J.M. Drummond, J. Henn, G.P. Korchemsky and E. Sokatchev, On planar gluon amplitudes/Wilson loops duality, Nucl. Phys. B 795 (2008) 52 [arXiv:0709.2368] [INSPIRE].

[67] J.M. Drummond, J. Henn, G.P. Korchemsky and E. Sokatchev, Conformal Ward identities for Wilson loops and a test of the duality with gluon amplitudes, Nucl. Phys. B 826 (2010) 337 [arXiv:0712.1223] [inSPIRE].

[68] L.J. Dixon, L. Magnea and G.F. Sterman, Universal structure of subleading infrared poles in gauge theory amplitudes, JHEP 08 (2008) 022 [arXiv:0805.3515] [INSPIRE].

[69] Z. Bern et al., The two-loop six-gluon MHV amplitude in maximally supersymmetric Yang-Mills theory, Phys. Rev. D 78 (2008) 045007 [arXiv: 0803.1465] [inSPIRE].

[70] J.M. Drummond, J. Henn, G.P. Korchemsky and E. Sokatchev, Hexagon Wilson loop = six-gluon MHV amplitude, Nucl. Phys. B 815 (2009) 142 [arXiv:0803.1466] [INSPIRE].

[71] L.F. Alday, D. Gaiotto and J. Maldacena, Thermodynamic bubble ansatz, JHEP 09 (2011) 032 [arXiv:0911.4708] [INSPIRE].

[72] N. Beisert, B. Eden and M. Staudacher, Transcendentality and crossing, J. Stat. Mech. 01 (2007) P01021 [hep-th/0610251] [INSPIRE].

[73] L.F. Alday, D. Gaiotto, J. Maldacena, A. Sever and P. Vieira, An operator product expansion for polygonal null Wilson loops, JHEP 04 (2011) 088 [arXiv: 1006.2788] [INSPIRE].

[74] E. Remiddi and J.A.M. Vermaseren, Harmonic polylogarithms, Int. J. Mod. Phys. A 15 (2000) 725 [hep-ph/9905237] [InSPIRE].

[75] S. Caron-Huot and S. He, Jumpstarting the all-loop S-matrix of planar $N=4$ super Yang-Mills, JHEP 07 (2012) 174 [arXiv:1112.1060] [INSPIRE].

[76] I.A. Korchemskaya and G.P. Korchemsky, On lightlike Wilson loops, Phys. Lett. B 287 (1992) 169 [InSPIRE].

[77] F.C.S. Brown, Polylogarithmes multiples uniformes en une variable (in French), Compt. Rend. Acad. Sci. Paris Ser. I 338 (2004) 527.

[78] J. Golden and M. Spradlin, An analytic result for the two-loop seven-point MHV amplitude in $N=4$ SYM, JHEP 08 (2014) 154 [arXiv:1406.2055] [INSPIRE].

[79] J.M. Drummond, G. Papathanasiou and M. Spradlin, A symbol of uniqueness: the cluster bootstrap for the 3-loop MHV heptagon, JHEP 03 (2015) 072 [arXiv: 1412.3763] [INSPIRE].

[80] L.J. Mason and D. Skinner, The complete planar S-matrix of $N=4$ SYM as a Wilson loop in twistor space, JHEP 12 (2010) 018 [arXiv: 1009.2225] [INSPIRE].

[81] S. Caron-Huot, Notes on the scattering amplitude/Wilson loop duality, JHEP 07 (2011) 058 [arXiv: 1010.1167] [INSPIRE].

[82] D. Binosi and L. Theussl, JaxoDraw: a graphical user interface for drawing Feynman diagrams, Comput. Phys. Commun. 161 (2004) 76 [hep-ph/0309015] [INSPIRE].

[83] D. Binosi, J. Collins, C. Kaufhold and L. Theussl, JaxoDraw: a graphical user interface for drawing Feynman diagrams. Version 2.0 release notes, Comput. Phys. Commun. 180 (2009) 1709 [arXiv:0811.4113] [INSPIRE].

[84] J.A.M. Vermaseren, Axodraw, Comput. Phys. Commun. 83 (1994) 45 [inSPIRE].

[85] C. Anastasiou, A. Brandhuber, P. Heslop, V.V. Khoze, B. Spence and G. Travaglini, Two-loop polygon Wilson loops in $N=4$ SYM, JHEP 05 (2009) 115 [arXiv:0902.2245] [INSPIRE]. 